\title{
POLIEDROS DE GRAFOS TRANSITIVOS
}

Maria Angela Melo de Campos Gurgel

TESE APRESENTADA

AO

INSTITUTO DE MATEMÁTICA E ESTATÍSTICA

DA

UNIVERSIDADE DE SÃO PAULO

PARA OBTENÇÃO DO GRAU DE DOUTOR

EM

MATEMÁTICA APLICADA

Área de concentração: Ciência da Computação

Orientadora: Profa. Dra. Yoshiko Wakabayashi

- São Paulo, outubro de 1992 - 
à minha màe Cloris.

ao meu marido Antonio Luiz e. aos nossos filhos Fernanda, Henrique e Thais. 


\section{AGRADECIMENTOS}

À Yoshiko, orientadora segura; minuciosa nos detalhes; que norteou meus passos pelo labirinto, como se conhecesse a saída;

Ao Professor Grötschel pelas sugestões que culminaram na obtenção das facetas semi-kcercas;

Ao Arnaldo cujas 'dicas' contribuíram para simplificar as demonstrações de alguns resultados do capítulo IV;

À "vó" Có, aquela que efetivamente me substituiu em tantos momentos;

Ao "papai" Gurgel, que tão sabiamente alimentou meu ego;

Aos meus filhos Fê, Rick e Tatá; esta é a prova de que nunca é tarde para a realização de um sonho;

Aos meus irmãos Gina. Nenê e Rosa, pelo companheirismo que nos une;

Aos amigos Humberta, Edna, Bete, Inês, Mariane, Bel, Saulo, Giraça, René, Lucinha. Nami e Yô, que presentes ou em pensamento, sempre estiveram comigo;

Aos meus ex-aluninhos Wania, Alan, Lúcia, Cris, Tito, Denise, Dilma e Carlinhos, por razões que ... só Freud explica;

Finalmente, aos meus professores e colegas do dia a dia. em especial ao Haroldo, que me ensinou a usar o xfig, muito obrigado a todos! 


\section{CONTEÚDO}

Introdução . . . . . . . . . . . . . . . . . . . . . . . . . 1

I. Conceitos Básicos . . . . . . . . . . . . . . . . . . . . . . . . 4

I.1 Teoria dos Grafos . . . . . . . . . . . . . . . . . . . . . . . . 4

I.2 Teoria dos Poliedros, Álgebra Linear e Programação Linear . . . . . . . 10

I.3 Teoria da Complexidade Computacional . . . . . . . . . . . . . . . 17

II. Problemas da Pré-Ordem Completa, da Pré-Ordem

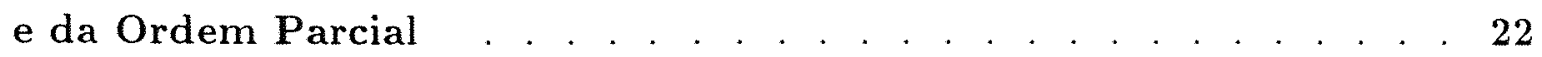

II.1 Os Problemas . . . . . . . . . . . . . . . . . . . . . . . . . . 23

II.2 Complexidade Computacional . . . . . . . . . . . . . . . . . . . 24

III. Politopo da Pré-Ordem Completa, da Pré-Ordem e da

Ordem Parcial: Dimensão e Estrutura Facial . . . . . . . . . . . . 35

III.1 Introdução . . . . . . . . . . . . . . . . . . . . . . . . . . . . 35

III.2 Definições e Resultados Básicos . . . . . . . . . . . . . . . . . . 36

III.3 Facetas . . . . . . . . . . . . . . . . . . . . . . . . . . 41

III.3.1 Facetas dos triângulos . . . . . . . . . . . . . . . . . . . 47

III.3.2 Facetas dos arcos paralelos . . . . . . . . . . . . . . . . . 49

III.3.3 Facetas triviais . . . . . . . . . . . . . . . . . . . . . . 51

III.3.4 Facetas dos circuitos ímpares com duas cordas . . . . . . . . . 52

III.3.5 Facetas dos pentágonos . . . . . . . . . . . . . . . . . . 59

III.3.6 Facetas dos heptágonos . . . . . . . . . . . . . . . . . . . 66 
III.3.7 Facetas das semi- $k$-cercas . . . . . . . . . . . . . . . . . 73

III.4 Relação entre os politopos . . . . . . . . . . . . . . . . . . . . 83

III.5 Soluçôes fracionárias . . . . . . . . . . . . . . . . . . . . . . . 85

IV. Diâmetro e Adjacência de Vértices do Politopo

da Pré-Ordem Completa . . . . . . . . . . . . . . . . . . . . . . 90 90

Anexo . . . . . . . . . . . . . . . . . . 103

Considerações finais . . . . . . . . . . . . . . . . . . . 125

Referências . . . . . . . . . . . . . . 126

Lista de Símbolos e Notação . . . . . . . . . . . . . . . . . . . . . 129

Índice de Definições . . . . . . . . . . . . . . . . . . . . . . . . . . 132 


\section{ABSTRACT}

Let $D_{n}=\left(V_{n}, A_{n}\right)$ be the complete digraph on $n$ vertices. An edge set $A \subseteq A_{n}$ is called a complete pre-order (pre-order, partial order) of $D_{n}$ if $H=\left(V_{n}, A\right)$ is a total transitive (transitive, anti-symmetric transitive) subgraph of $D_{n}$.

Given a digraph $D_{n}=\left(V_{n}, A_{n}\right)$ with a weight $p_{e} \in \mathbb{Z}$ assigned to each edge $e \in A_{n}$, the problem of finding a complete pre-order (pre-order, partial order) $A \subseteq A_{n}$ such that $p(A):=\sum_{e \in A} p_{e}$ is as large as possible is called the complete pre-order (pre-order, partial order) problem.

We study the polytopes associated with each of these problems, defined as the convex hull of the incidence vectors of all complete pre-orders (pre-orders, partial orders) of $D_{n}$. We present several classes of inequalities and investigate which class is facet-defining, valid or nonvalid for each of these polytopes. We study the adjacency relation on the vertices of the complete pre-order polytope and prove that the diameter of this polytope equals two. We also show that the three problems considered are INIP-hard.

\section{RESUMO}

Seja $D_{n}=\left(V_{n}, A_{n}\right)$ o digrafo completo com $n$ vértices. Um subconjunto de arestas $A \subseteq A_{n}$ é chamado uma pré-ordem completa (pré-ordem, ordem parcial) de $D_{n}$ se $H=\left(V_{n}, A\right)$ é um subgrafo transitivo total (transitivo, anti-simétrico transitivo) de $D_{n}$. Considerando uma função peso $p: A_{n} \rightarrow \mathbb{Z}$, o problema de encontrar uma pré-ordem completa (préordem, ordem parcial) $A \subseteq A_{n}$ de peso máximo, sendo $p(A)=\sum_{e \in A} p_{e}$, é chamado problema da pré-ordem completa (pré-ordem, ordem parcial).

Estudamos os politopos associados a cada um destes problemas, definidos como o fecho convexo dos vetores de incidência de todas as pré-ordens completas, pré-ordens e ordens parciais de $D_{n}$. Apresentamos várias classes de inequações e investigamos quais delas definem facetas para cada um desses politopos. Estudamos a relação de adjacência de vértices no politopo da pré-ordem completa e provamos que o diâmetro deste politopo é 2. Também mostramos que os três problemas considerados são INIP-difíceis. 


\section{Introdução}

Neste trabalho são estudados, sob uma abordagem poliédrica, três problemas de otimização combinatória: o problema da pré-ordem completa, o da pré-ordem e o da ordem parcial. Cada um destes problemas tem como instância um digrafo completo $D_{n}=\left(V_{n}, A_{n}\right)$ com $n$ vértices e uma função peso $p: A_{n} \rightarrow \mathbb{Z}$, definida nas arestas de $D_{n}$, e tem como objetivo encontrar um subdigrafo $H$ de $D_{n}$ com certas propriedades especiais, que seja de peso máximo. Mais precisamente, no problema da pré-ordem completa procura-se em $D_{n}$ um subdigrafo gerador transitivo e total $H=(V H, A H)$, tal que $p(A H)=\sum_{a \in A H} p(a)$ seja máximo. No problema da pré-ordem, o subdigrafo $H$ deve ser transitivo; e no da ordem parcial, $H$ deve ser transitivo e anti-simétrico.

Os três problemas referidos são INIP-difíceis. Assim, a razão de se estudá-los sob o enfoque poliédrico foi dupla: por um lado, um interesse puramente teórico em "desvendar facetas" de poliedros associados a problemas difíceis; por outro lado, a esperança de que tais resultados teóricos poderão ser usados no desenvolvimento de algoritmos baseados em planos-de-corte e "branch and bound", que sejam úteis na solução de instâncias reais. 
Nosso estudo consistiu em definir para cada um dos problemas, um politopo (poliedro limitado) cujos vértices estão em correspondência um a um com as possíveis soluções do problema, e investigar a estrutura facial deste politopo.

O objeto inicial de nossa pesquisa foi o politopo da pré-ordem completa. Ao longo do desenvolvimento deste estudo, surgiram porém, várias classes de inequações que nos levaram a indagar se também seriam facetas do politopo da pré-ordem e da ordem parcial. Assim, de maneira natural, passamos a estudar os três politopos. Isto trouxe, de certa forma, uma unidade ao trabalho, já que há nos três problemas um elo comum que é a propriedade transitiva.

Investigando na literatura, descobrimos que dentre os problemas deste tipo, onde a transitividade é exigida, estes três eram os únicos ainda não estudados sob o enfoque poliédrico. Estes resultados, contribuíram assim, para completar o estudo da classe dos politopos associados a problemas sobre digrafos onde se procura subdigrafos com a propriedade transitiva, combinada (ou não) com outras propriedades como a total, simétrica e anti-simétrica.

A seguir, apresentamos um breve resumo do conteúdo de cada capítulo.

No capítulo I, revemos resultados fundamentais e definç̧ôes básicas da teoria dos grafos, de programação linear, dos poliedros e da complexidade computacional. Aqui, reunimos quase todas as definições utilizadas ao longo da dissertação e estabelecemos a notação adotada. Os conceitos novos introduzidos neste capítulo, que merecem destaque, são os de digrafos totais, transitivos, simétricos ou anti-simétricos, conforme a relação binária sobre os vértices, definida pelo conjunto de suas arestas, seja de um desses tipos.

No capítulo II, introduzimos os problemas da pré-ordem completa, da pré-ordem $\mathrm{e}$ da ordem parcial. Provas de que estes problemas são INIP-difíceis são aqui apresentadas.

O capítulo III contém a parte central da nossa pesquisa. Após definir os politopos associados aos problemas em estudo e provar que eles têm dimensão completa, apresen- 
tamos o lema da elevaçấo, usado em quase todas as demonstrações posteriores, para se estender inequações-facetas de politopos de certa dimensão para poliedros de dimensões maiores. O restante deste capítulo apresenta grandes classes de inequações-facetas dos politopos $P_{P O C}, P_{P O}$ e $P_{O P}$, associados aos problemas da pré-ordem completa, da préordem e da ordem parcial, respectivamente. Mostramos que algumas dessas classes são de inequações que definem facetas apenas de $P_{P O C}$, como é o caso das inequações das semi-k-cercas, das inequaçôes-pentágonos e das inequações-heptágonos. Por outro lado, exibimos também inequações como a dos $k$-circuitos ímpares com 2-cordas que são inequações-facetas apenas de $P_{P O}$ e de $P_{O P}$. Para cada classe de inequações apresentada, fazemos um estudo procurando relacioná-la com cada um dos três politopos considerados. Neste aspecto, conforme pode ser observado no decorrer deste capítulo, ainda existem algumas questões não respondidas.

O capítulo IV contém a prova de que o diâmetro de $P_{P O C}$ é 2 . A determinação dos diâmetros de $P_{P O}$ e $P_{O P}$ é um problema teórico interessante, em aberto.

$\mathrm{O}$ anexo contém apenas a prova da validade da inequação-heptágono para $P_{P O C}$. Este resultado, que deveria ser parte do capítulo III, encontra-se no anexo por se tratar de uma demonstração longa e exaustiva que poderia comprometer a continuidade dos resultados apresentados nesse capítulo. 


\section{Capítulo I}

\section{Conceitos Básicos}

Conceitos matemáticos básicos que serão usados no decorrer deste trabalho são introduzidos neste capítulo. Em cada seção, referências a textos apropriados serão feitas, sempre que necessário. Estes conceitos vêm geralmente da teoria dos grafos, teoria dos poliedros, programação linear e teoria da complexidade computacional.

\section{I.1 Teoria dos Grafos}

Como os problemas que estudamos são de otimização em grafos e não existe uma terminologia comum na teoria dos grafos para todos os conceitos que usamos, adotamos as definições que consideramos mais apropriadas para este trabalho. A maioria dos conceitos aqui definidos podem ser encontrados, com variação de notação, em Berge [1973], Bondy e Murty [1976], Lucchesi [1979] e Szwarcfiter [1984].

Um digrafo ou grafo dirigido $D=(V, A)$ consiste de um conjunto finito não vazio $V$ 
de elementos denominados vértices e um conjunto $A$ de elementos denominados arestas, que são pares ordenados de vértices, isto é, $A \subseteq\{(u, v): u, v \in V\}$. Se $\alpha=(u, v)$ é uma aresta de um digrafo $D=(V, A)$, dizemos que $\alpha$ vai de $u$ para $v$, incide em $u$ e em $v$, e que $u$ e $v$ são extremos de $\alpha$. Também nos referimos a $u$ como início e a $v$ como término da aresta $\alpha$.

Dois vértices $u$ e $v$ de um digrafo $D=(V, A)$ são adjacentes se $(u, v)$ ou $(v, u) \in$ $A$; uma aresta de $D$ é um laço se seus extremos são iguais. Digrafos sem laços são denominados simples. A maioria dos digrafos que ocorrem neste trabalho são simples.

Algumas vezes, por simplicidade de notação, uma aresta $\alpha=(u, v)$ de um digrafo $D$ será denotada sem os parênteses ou mesmo sem os parênteses e a vírgula. Por exemplo, se $\alpha=(u, v)$ subscreve uma variável $x$, usaremos uma das notações $x_{\alpha}, x_{(u, v)}, x_{u, v}$ ou $x_{u v}$, dependendo da conveniência dentro do contexto.

Se $W$ é um subconjunto de vértices de um digrafo $D=(V, A)$, denotamos por $I(W)$ o subconjunto das arestas de $D$ com ambos os extremos em $W$ e por $\delta(W)$ o subconjunto das arestas de $D$ com um extremo em $W$ e o outro em $V \backslash W$, isto é,

$$
\begin{aligned}
& \mathrm{I}(\mathbf{W}):=\{(u, v) \in A: u, v \in W\} \quad \mathrm{e} \\
& \delta(\mathbf{W}):=A \backslash(I(W) \cup I(V \backslash W)) .
\end{aligned}
$$

No caso em que todas as arestas de $\delta(W)$ possuem início em $W$ ou todas possuem término em $W, \delta(W)$ é denominado corte orientado; caso contrário é simplesmente um corte. Para cada $v \in V, \delta(v)$ abrevia $\delta(\{v\})$. Se $\delta(v)$ for um corte orientado, então $v$ será denominado fonte (sorvedouro) se as arestas de $\delta(v)$ tiverem seu início (término) em $v$.

Se os digrafos $D=(V, A)$ e $G=(W, B)$ são tais que $W \subseteq V$ e $B \subseteq A$, então $G$ é dito um subdigrafo de $D$. Neste caso, dizemos que $D$ contém $G$ ou que $G$ está contido em $D$ (denota-se por $G \subseteq D$ ). Se $E \subseteq A$, então o subdigrafo de $D$ com conjunto de arestas $E$ e vértices consistindo dos extremos das arestas de $E$ é o subdigrafo gerado por $\mathbf{E}$ e 
denotado por $\mathbf{D}[\mathbf{E}]$. Se $W \subseteq V$, então $G=(W, I(W))$ é o subdigrafo gerado por $W$ e denotado por $\mathbf{D}[\mathbf{W}]$. Por simplicidade de notação, especialmente no capítulo III, também denotaremos por [X] o subdigrafo gerado por $X$, se $X \subseteq V$ ou $X \subseteq A$.

Se $W$ é um subconjunto de vértices de um digrafo $D=(V, A)$, então $\mathbf{D}-\mathbf{W}$ denota o subdigrafo obtido de $D$, removendo-se $W$, definido com conjunto de vértices $V \backslash W$ e $\operatorname{arestas} I(V \backslash W)$. Observe que $D-W=D[V \backslash W]$. Escrevemos $\mathbf{D}-\mathbf{v}$ para denotar o subdigrafo $D-\{v\}$, se $v$ é um vértice de $D$.

Dois digrafos $D=(V, A)$ e $G=(W, B)$ são isomorfos se existe uma bijeção $\varphi$ : $V \rightarrow W$ tal que para $u, v \in V$, tem-se que $(u, v) \in A$ se e somente se $(\varphi(u), \varphi(v)) \in B$ Escrevemos $G \cong D$ para indicar que $G$ e $D$ são isomorfos. Dizemos que um digrafo $D=(V, A)$ é completo se $A=V \times V \backslash\{(u, u): u \in V\}$. Para todo natural $n$, existe , a menos de isomorfismo, um único digrafo completo com $n$ vértices, que será, em geral, denotado por $\mathbf{D}_{\mathbf{n}}=\left(\mathrm{V}_{\mathrm{n}}, \mathbf{A}_{\mathbf{n}}\right)$.

Se $\alpha=(u, v)$ é uma aresta de $D_{n}, \bar{\alpha}$ denota a aresta $(v, u)$, reversa à $\alpha$. Generalizando este conceito, se $B \subseteq A_{n}$, então $\overline{\mathbf{B}}$, chamado reverso de $B$, denota o conjunto das arestas reversas às de $B$.

Um clique em um digrafo $D=(V, A)$ é um subdigrafo completo de $D$, não necessariamente maximal.

Uma seqüência alternada não nula $S=<v_{1}, \alpha_{1}, v_{2}, \alpha_{2}, \ldots, \alpha_{k-1}, v_{k}>$ é um passeio em um digrafo $D=(V, A)$ se $v_{i} \in V(1 \leq i \leq k), \alpha_{i} \in A$ e os vértices $v_{i}$ e $v_{i+1}$ são extremos da aresta $\alpha_{i}(1 \leq i \leq k-1)$. Se a seqüencia $S$ acima é um passeio, então dizemos que é um passeio de $v_{1}$ a $v_{k}$ ou um $\left[v_{1}-v_{k}\right]$-passeio. O comprimento de um tal passeio é $k-1$. No caso em que $v_{i} \neq v_{j}$ para $i \neq j(1 \leq i, j \leq k), S$ é um caminho entre $v_{1}$ e $v_{k}$. Se a seqüência $S$ é um $\left[v_{1}-v_{k}\right]$-caminho, ele é dirigido de $v_{1}$ para $v_{k}$ ou é um caminho dirigido se $\alpha_{i}=\left(v_{i}, v_{i+1}\right)$ para $1 \leq i \leq k-1$. Neste caso, o caminho dirigido de $v_{1}$ para $v_{k}$ pode ser denotado pela seqüencia de seus vértices, isto é, por $\left\langle v_{1}, \ldots, v_{k}\right\rangle$. Se 
$v_{1}=v_{k}$ e $v_{1}, \ldots, v_{k-1}$ forem todos distintos, então $S$ é um circuito. O circuito é dirigido se todas as suas arestas estiverem orientadas num mesmo sentido. Neste caso, o circuito pode ser denotado pela seqüência de seus vértices, ou de suas arestas. Um passeio de comprimento $k$ é um k-passeio; um $k$-passeio é par ou ímpar, conforme $k$ seja par ou ímpar. Um 3-circuito é denominado um triângulo. Freqüentemente usamos os termos caminho, circuito ou triângulo para denotar o digrafo correspondente a um caminho, um circuito ou um triângulo, respectivamente.

Se $D$ é um digrafo com $n$ vértices $(n \geq 1)$, um $n$-caminho dirigido em $D$ é um caminho hamiltoniano e um $n$-circuito dirigido é um circuito hamiltoniano. Um digrafo é hamiltoniano se contém um circuito hamiltoniano.

Se $C=\langle 1,2, \ldots, k\rangle$ é um $k$-circuito dirigido com vértices $1,2, \ldots, k, k \geq 5$, então o conjunto $\{(i,(i+2) \bmod k): i=1, \ldots, k\}$ é denominado o conjunto das 2 -cordas de $C$ e o conjunto $\{(i,(i+3) \bmod k): i=1, \ldots, k\}$ é o conjunto das 3 -cordas de $C$.

Um digrafo $D=(V, A)$ é conexo se existe um caminho entre quaisquer dois vértices de $D$; caso contrário, $D$ é desconexo e denominamos componentes os subdigrafos conexos maximais de $D$. Dizemos que $D$ é fortemente conexo se para quaisquer dois vértices $u, v \in V$, existe um $[u-v]$ e um $[v-u]$-caminhos dirigidos em $D$. Subdigrafos maximais fortemente conexos de um digrafo $D$ sào denominados componentes fortemente conexos do digrafo.

Sejam $D_{1}, D_{2}, \ldots, D_{m}$ os componentes fortemente conexos de um digrafo $D$. O digrafo condensado de $D$ (denotado por $\widehat{D}$ ) é um digrafo com $m$ vértices $v_{1}, v_{2}, \ldots, v_{m}$; existe uma aresta em $\widehat{D}$ que vai de $v_{i}$ para $v_{j}(i \neq j)$ se e somente se existe uma aresta em $D$, com início em $V D_{i}$ e término em $V D_{j}$.

Um subdigrafo $G=(W, B)$ de um digrafo $D=(V, A)$ é gerador se $|W|=|V|$.

Digrafos acíclicos sào aqueles que nào contêm circuitos dirigidos.

Um digrafo $D=(V, A)$ é um torneio se, para quaisquer vértices $u, v \in V, u \neq v$, 
exatamente uma das arestas com extremos $u$ e $v$ está em $A$.

Com a finalidade de definirmos grafos especiais que serão investigados neste trabalho, estabelecemos aqui algumas relações binárias $R$ sobre $S(R \subseteq S \times S)$. Em outros textos, as definições das relações transitiva e total podem ser ligeiramente diferentes. Dizemos que:

- $R$ é simétrica se $(n, m) \in R \Rightarrow(m, n) \in R, \forall n, m \in S$;

- $R$ é anti-simétrica se $(n, m) \in R$ e $(m, n) \in R \Rightarrow n=m, \quad \forall n, m \in S^{\prime}$;

- $R$ é transitiva se $(n, m) \in R \quad$ e $(m, s) \in R \Rightarrow(n, s) \in R, \quad \forall n, m, s \in S$, $n \neq m \neq s \neq n$

- $R$ é total se $(n, m) \in R \quad$ ou $(m, n) \in R, \quad \forall n, m \in S, n \neq m$.

Uma relação binária é uma:

- pré-ordem se é transitiva;

- pré-ordem completa se é transitiva e total;

- ordem parcial se é anti-simétrica e transitiva;

- ordem linear se é anti-simétrica, transitiva e total.

Note que, dado um digrafo $D=(V, A)$, o conjunto de arestas $A$ é uma relação binária sobre $V$. Um tal digrafo é denominado total, simétrico, anti-simétrico ou transitivo, conforme $A$ seja uma relação total, simétrica, anti-simétrica ou transitiva, respectivamente.

Seja $D=(V, A)$ um digrafo e $B \subseteq A$. Então dizemos que $B$ é uma pré-ordem (ordem parcial, ordem linear, pré-ordem completa) de $\mathrm{D}$, se $B$ é uma pré-ordem (ordem parcial, ordem linear, pré-ordem completa) sobre $V$.

Se $D=(V, A)$ é um digrafo e se $B \subseteq A$ é uma ordem linear de $D$, existe um único caminho hamiltoniano em $D$, cujas arestas estão contidas em $B$. A seqüuência de vértices 
deste caminho, digamos $O:=<u_{1}, \ldots, u_{n}>$, é uma outra forma de nos referirmos à ordem linear $B$. Dizemos que tal ordem linear $B$ é definida por $O$, e a denotamos também por $\mathbf{A}(\mathbf{O})$. Note que

$$
\mathbf{A}(\mathbf{O})=\left\{\left(u_{i}, u_{j}\right): 1 \leq i<j \leq n\right\}
$$

O fecho transitivo de um digrafo $G$ é o menor digrafo transitivo que contém $G$.

Um emparelhamento em um digrafo $D=(V, A)$ é um subconjunto de arestas de $D$, distintas de laços, tal que quaisquer duas arestas não possuem um extremo em comum. Dizemos que un emparelhamento $E \subseteq A$ é perfeito se tocio veruce de $D$ e extremo de alguma aresta em $E$ e é um k-emparelhamento se $|E|=k, k \geq 1$.

Um grafo $G=(V, A)$ consiste de um conjunto finito não vazio $V$ de elementos denominados vértices e um conjunto de pares não ordenados de elementos de $V$, denominados arestas, isto é, $A \subseteq\{\{u, v\}: u, v \in V\}$.

Como vemos, a única diferença entre grafos e digrafos consiste na orientação das arestas. Todos os conceitos que não envolvem a noção de orientação se aplicam automaticamente para grafos.

Grafos acíclicos são aqueles que nâo contêm circuitos.

Um $n$-caminho em um grafo com $n$ vértices é um caminho hamiltoniano e um $n$ circuito um circuito hamiltoniano. Um grafo hamiltoniano é um grafo que contém um circuito hamiltoniano.

Para cada natural $n$, existe, a menos de isomorfismo, um único grafo completo com $n$ vértices, denotado por $K_{n}=\left(V_{n n}, A_{n}\right)$.

A distância $\mathrm{d}(\mathrm{u}, \mathrm{v})$ entre dois vértices quaisquer $u$ e $v$ de um grafo $G=(V, A)$ é o comprimento de um caminho mais curto (isto é, de menor comprimento) entre $u$ e $v$, se existir; caso contrário, $d(u, v)=\infty$. O diâmetro de $G$, denotado por $\operatorname{diam}(\mathbf{G})$ é a maior distância entre dois vértices quaisquer de $G$.

Como a maioria dos grafos que ocorrem neste trabalho são dirigidos, às vezes usaremos 
os termos grafo e subgrafo para denotar um digrafo e subdigrafo, respectivamente. Isto simplificará nossa notação e não causará confusão ao leitor atento.

Dado um grafo (digrafo) $H$, a menos de menção em contrário, VH e AH denotam o conjunto dos vértices e das arestas de $H$, respectivamente. Também nos referimos a AP e VP como o conjunto das arestas e dos vértices de um passeio $P$ em um grafo (digrafo), respectivamente.

Diferenças simétricas de subconjuntos de arestas ou de vértices de um grafo (digrafo) são às vezes mencionadas. Se $A$ e $B$ são conjuntos, $\mathbf{A} \Delta \mathbf{B}$ denota a diferença simétrica de $A$ e $B$, isto é, $A \Delta B:=(A \backslash B) \cup(B \backslash A)$.

\section{I.2 Teoria dos Poliedros, Álgebra Linear e Programação Linear}

Os conceitos aqui apresentados são usados freqüentemente no capítulo III, onde analisamos a estrutura facial dos poliedros que associamos aos problemas que são objetos de estudo deste trabalho.

Denotamos por $\mathbb{R}, \mathbb{Z}$ e $\mathbb{N}$ os conjuntos dos números reais, inteiros e naturais, respectivamente. O conjunto dos reais não negativos será denotado por $\mathbb{R}^{*}$. O conjunto dos vetores $n$-dimensionais com componentes em um conjunto qualquer $W$ será denotado por $W^{n}$, sendo $n \in \mathbb{N}, n>0$. Assim, $\mathbb{R}^{n}\left(\mathbb{Z}^{n}\right)$ denota o conjunto de todos os vetores com $n$ componentes reais (inteiras). Ampliando esta notação, $\mathbb{R}^{m \times n}\left(\mathbb{Z}^{m \times n}\right)$ com $m, n>0$, $m, n \in \mathbb{N}$, denota o conjunto das matrizes reais (inteiras), com $m$ linhas e $n$ colunas.

Se $E$ é um conjunto finito ordenado e $W$ um conjunto qualquer, $\mathrm{W}^{\mathrm{E}}$ denota o conjunto dos vetores com $|E|$ componentes indexadas pelos elementos de $E$, com entradas em $W$. Similarmente, se $A$ é uma matriz, $A \in \mathbb{R}^{E \times F}$ significa que $A$ é real, possui $|E|$ linhas e $|F|$ colunas, indexadas pelos elementos dos conjuntos finitos ordenados $E$ e $F$, respectivamente.

Para um número real $x,\lfloor x\rfloor$ denota o maior inteiro menor ou igual a $x$. A menos 
de especificação em contrário, vetores serão vetores coluna. Assim, se $x$ é um vetor, $x^{T}$ denota seu transposto. Um vetor ou uma matriz de zeros será denotada por 0 e um vetor de uns por 1. Um vetor $\left(x_{1}, x_{2}, \ldots, x_{n}\right)$ é referido como unitário se $\exists i, \quad 1 \leq i \leq n$, tal que $x_{i}=1$ e $x_{j}=0$ para $j \neq i$.

Se $E$ é um conjunto finito ordenado e $A \subseteq E$, denotamos por $\mathrm{x}^{A}$ o vetor de incidência ou vetor característico de $A$, definido como:

$$
x^{A}=\left(x_{e}^{A}, e \in E\right), \text { sendo } x_{e}^{A}=\left\{\begin{array}{ll}
1, & \text { se } e \in A \\
0, & \text { se } e \in E \backslash A
\end{array} .\right.
$$

Ao longo deste trabalho, identificamos funções $p: A \rightarrow \mathbb{R}$ com vetores em $\mathbb{R}^{A}$. Assim, se $a \in A, p_{a}$ pode ser a componente do vetor $p$ indexada por $a \in A$, ou o valor $p(a)$ da função $p$. Se $B \subseteq A$ e $p: A \rightarrow \mathbb{R}$ (ou $p \in \mathbb{R}^{A}$ ), então $p(B)$ denota a seguinte soma:

$$
p(B):=\sum_{b \in B} p_{b}
$$

Um vetor $x \in \mathbb{R}^{n}$ é uma combinação linear dos vetores $x_{1}, x_{2}, \ldots, x_{t} \in \mathbb{R}^{n}$, se, para algum $\alpha=\left(\alpha_{1}, \ldots, \alpha_{t}\right) \in \mathbb{R}^{t}$,

$$
x=\sum_{i=1}^{t} \alpha_{i} x_{i}
$$

Uma tal combinação linear é chamada

$$
\begin{aligned}
& \text { afim, se } \alpha_{1}+\cdots+\alpha_{t}=1, \\
& \text { cônica, se } \alpha_{1}, \cdots, \alpha_{t} \geq 0
\end{aligned}
$$

convexa, se for afim e cônica.

Para um conjunto não vazio $S \subseteq \mathbb{R}^{n}$, denotamos por lin $(\mathbf{S})(\operatorname{cone}(\mathbf{S})$, aff $(\mathbf{S})$, conv (S)) o fecho linear (cônico, afim, convexo) dos elementos de $S$, isto é, o conjunto 
de todos os vetores que são combinação linear (cônica, afim, convexa) de um número finito de vetores de $S$, respectivamente. Se $S$ for o conjunto vazio, então $\operatorname{lin}(S):=$ cone $(S):=$ $\{0\}$ e $\operatorname{conv}(S):=\operatorname{aff}(S):=\emptyset$. Um conjunto $S \subseteq \mathbb{R}^{n}$ é um subespaço linear (cone, subespaço afim, conjunto convexo) se $S=\operatorname{lin}(S)(S=\operatorname{cone}(S), S=\operatorname{aff}(S), S=$ $\operatorname{conv}(S))$.

Um subconjunto $S \subseteq \mathbb{R}^{n}$ é linearmente independente (independente afim) se para qualquer subconjunto finito $\left\{x_{1}, \ldots, x_{t}\right\}$ de $S, \sum_{i=1}^{t} \alpha_{i} x_{i}=0, \alpha_{i} \in \mathbb{R}\left(\sum_{i=1}^{t} \alpha_{i} x_{i}=0\right.$ e $\sum_{i=1}^{t} \alpha_{i}=0, \alpha_{i} \in \mathbb{R}$ ) implica $\alpha_{1}=\cdots=\alpha_{t}=0$ (implicam $\alpha_{1}=\cdots=\alpha_{t}=0$ ); caso contrário, $S$ é linearmente dependente (dependente afim). Para $S \subseteq \mathbb{R}^{n}$, o posto (posto afim) de $S$, denotado por posto (S) (posto a(S)) é a cardinalidade de um maior subconjunto de $S$ que é linearmente independente (independente afim). Observe que $\{0\}$ é independente afim, mas não é linearmente independente. Por outro lado, se $S$ é um conjunto linearmente independente, então $S$ é independente afim. Prova-se que para todo $S \subseteq \mathbb{R}^{n}$, se $0 \in$ aff $(S)$, então posto $\mathrm{a}(S)=$ posto $(S)+1$; e se $0 \notin$ aff $(S)$, então posto $\mathrm{a}(S)=$ posto $(S)$.

O posto de uma matriz $A$, denotado por posto (A), é o posto do conjunto de vetores coluna de $A$, que é igual ao posto do conjunto de vetores linha de $A$. Uma matriz $A \in \mathbb{R}^{m \times n}$ tem posto linha completo (posto coluna completo) se posto $(A)=m$ $($ posto $(A)=n)$.

Se $S \subseteq \mathbb{R}^{n}$, então a dimensão de $S$ (denotada por $\operatorname{dim}(\mathbf{S})$ ou $\operatorname{dim} \mathrm{S}$ ), é definida como

$$
\operatorname{dim}(S):=\text { posto } a(S)-1
$$

Um poliedro $P \subseteq \mathbb{R}^{n}$ é o conjunto solução de um sistema de inequações lineares, isto é,

$$
P=\left\{x \in \mathbb{R}^{n} \mid A x \leq b\right\}
$$

para uma matriz $A \in \mathbb{R}^{m \times n}$ e um vetor $b \in \mathbb{R}^{m}$. 
Se $a \in \mathbb{R}^{n} \backslash\{0\}$ e $a_{0} \in \mathbb{R}$, então o poliedro $\left\{x \in \mathbb{R}^{n}: a^{T} x \leq a_{0}\right\}$ é denominado semi-espaço e o poliedro $\left\{x \in \mathbb{R}^{n}: a^{T} x=a_{0}\right\}$ é denominado hiperplano. Para simplificar a notação, nos referimos ao semi-espaço $a^{T} x \leq a_{0}$ ou ao hiperplano $a^{T} x=a_{0}$. Observe que todo poliedro $P \subset \mathbb{R}^{n}$ é a intersecção de um número finito de semi-espaços do $\mathbb{R}^{n}$.

Um conjunto de vetores é um politopo se for o fecho convexo de um conjunto finito de vetores. É intuitivo que os conceitos de poliedro e politopo estão relacionados. Isto pode ser observado mais precisamente no teorema de decomposição de poliedros (Motzkin [1936]) e no corolário conhecido como teorema de base finita para politopos (Minkowski [1896], Steinitz [1916], Weyl [1935]).

Teorema I.1 (decomposição de poliedros): $P \subseteq \mathbb{R}^{n}$ é um poliedro se e somente se existem subconjuntos $V$ e $E$ em $\mathbb{R}^{n}$ tal que $P=\operatorname{conv}(V)+\operatorname{cone}(E)$.

Teorema I.2 (base finita para politopos): Um conjunto $P$ é um politopo se e somente se $P$ é um poliedro limitado.

O resultado abaixo mostra como a dimensào de um poliedro pode ser determinada.

Teorema I.3: Se $P \subseteq \mathbb{R}^{n}$ é um poliedro $e$ aff $(P)=\left\{x \in \mathbb{R}^{n}: C x=d\right\}$ para alguma matriz $C \in \mathbb{R}^{m \times n}$ ed $\in \mathbb{R}^{m}$, entâo $\operatorname{dim}(P)=n-$ posto $(C)$.

Dizemos que um poliedro $P \subseteq \mathbb{R}^{n}$ tem dimensão completa se $\operatorname{dim}(P)=n$; neste caso, não existe equação $a^{T} x=a_{0}, \operatorname{com} a \in \mathbb{R}^{n} \backslash\{0\}, a_{0} \in \mathbb{R}$, tal que $P \subseteq\left\{x \in \mathbb{R}^{n}\right.$ : $\left.a^{T} x=a_{0}\right\}$.

Uma inequação $a^{T} x \leq a_{0}$ com $a \in \mathbb{R}^{n}, a_{0} \in \mathbb{R}$, é válida para um poliedro $P \subseteq \mathbb{R}^{n}$, se $P \subseteq\left\{x: a^{T} x \leq a_{0}\right\}$. Um conjunto $F \subseteq P$ é uma face de $P$ se existe uma inequação válida $a^{T} x \leq a_{0}$ para $P$ tal que $F=\left\{x \in P: a^{T} x=a_{0}\right\}$. Neste caso, dizemos que $F$ é 
a face definida ou induzida por $a^{T} x \leq a_{0}$. Observe que, em particular, $P$ é face de $P$, considerando a inequação $0 x \leq 0$; as demais faces serão denominadas faces próprias.

Faces próprias não vazias maximais ou minimais com relação à inclusão são de extrema importância na investigação de um poliedro. A seguir, descrevemos estas faces e apresentamos alguns resultados que serão usados no decorrer deste trabalho.

Uma face de um poliedro $P$ consistindo de um único ponto é um vértice. Se $P$ for um politopo, usando o resultado abaixo, prova-se que $P$ é o fecho convexo de seus vértices.

Teorema I.4: Seja $x$ um ponto do poliedro $P \subseteq \mathbb{R}^{n}$. São equivalentes:

(i) $x$ é um vértice de $P$;

(ii) existe um vetor $c \in \mathbb{R}^{n}$ tal que $x$ é o único vértice de $P$ que maximiza $c^{T} x$, i.é, $c^{T} x>c^{T} y$ para todo $y \in P ;$

(iii) $x \notin \operatorname{conv}(P-\{x\})$.

Dizemos que dois vértices de um poliedro $P$ são adjacentes, se existe uma face de dimensão 1 (denominada aresta) que os contém.

O resultado abaixo contém algumas caracterizações de adjacência em um politopo.

Teorema I.5: Seja $P \subset \mathbb{R}^{n}$ um politopo com conjunto de vértices $V$. Sejam $x$ e y vértices distintos de P. Sâo equivalentes:

(i) $x$ e y são adjacentes;

(ii) $z \notin \operatorname{conv}(P-\{x, y\})$, para todo $z$, tal que $z=\lambda x+(1-\lambda) y, \quad 0<\lambda<1$;

(iii) todo $z$, tal que $z=\lambda x+(1-\lambda) y, 0<\lambda<1$, pode ser escrito de forma única como combinação convexa dos vértices de $V$;

(iv) existe um vetor $c \in \mathbb{R}^{n}$ tal que $x$ e y sâo os únicos vértices que maximizam $c^{T} x$ sobre $P$. 
Uma face própria de um poliedro $P$ é uma faceta se for não vazia e não estiver contida em nenhuma outra face própria de $P$, ou seja, é uma face não vazia cuja dimensão é igual a $\operatorname{dim}(P)-1$. A inequação que define uma faceta é referida como uma inequação-faceta. Muitas vezes, por simplicidade, dizemos que uma inequação é faceta de um poliedro, devendo ficar subentendido que se trata de uma inequação-faceta.

O teorema enunciado a seguir caracteriza inequações-facetas.

Teorema I.6: Seja $P \subseteq \mathbb{R}^{n}$ um poliedro e $F$ uma face náa vazia de $P, F=\{x \in P$ : $\left.a^{T} x=a_{0}\right\}$. São equivalentes:

(i) F é uma faceta de $P$;

(ii) $\operatorname{dim}(F)=\operatorname{dim}(P)-1$;

(iii) se aff $(P)=\left\{x \in \mathbb{R}^{n}: C x=d\right\}$ (sendo $C x=d$ um sistema minimal de equações) e $F \subseteq\left\{x \in P: b^{T} x=b_{0}\right\}$, sendo $b^{T} x \leq b_{0}$ uma inequação-faceta de $P$, então $\exists \lambda \in \mathbb{R}^{n-\operatorname{dim}(P)}$ e $\alpha \in \mathbb{R}^{*}$, tal que $b^{T}=\lambda^{T} C+\alpha a^{T} ;$

(iv) se $P$ tem dimensâa completa e $F \subseteq\left\{x \in P: b^{T} x=b_{0}\right\}$, sendo $b^{T} x \leq b_{0}$ uma inequação-faceta de $P$, entấo existe $\alpha \in \mathbb{R}^{*}$, tal que $b^{T}=\alpha a^{T}$.

Note que se um poliedro tem dimensão completa, o resultado acima diz que para cada faceta existe uma única inequação que a define, a menos de multiplicação por reais nãonegativos. Além disso, o teorema sugere duas formas para se provar que uma inequação $a^{T} x \leq a_{0}$, válida para um poliedro $P$ de dimensão completa, é uma inequação faceta. Ou exibimos $\operatorname{dim}(P)$ vetores independentes afim que satisfazem $a^{T} x \leq a_{0}$ com igualdade, ou consideramos

$$
F_{a}=\left\{x \in P: a^{T} x=a_{0}\right\} \subseteq F_{b}=\left\{x \in P: b^{T} x=b_{0}\right\},
$$

sendo $F_{b}$ faceta de $P$ e provamos os itens (i) e (ii) abaixo: 
(i) $F_{a} \neq \emptyset$;

(ii) existe $\alpha \in \mathbb{R}^{*}$ tal que $a^{T}=\alpha b^{T}$.

Freqëntemente usaremos este segundo método no capítulo III, omitindo a prova do item (i), por ser este um fato trivial nas demonstrações.

Dado um politopo $P$, o esqueleto de $P$ é definido como o grafo $G(P)$, tal que: $G(P)$ tem como conjunto de vértices o conjunto dos vértices de $P$ e dois vértices $u$ e $v$ em $G(P)$ são ligados por uma aresta se e somente se $u$ e $v$ são adjacentes em $P$. $O$ diâmetro de um politopo $P$, referido como diam $(\mathbf{P})$, é o diâmetro do esqueleto de $P$.

O problema de maximizar ou minimizar uma função linear sobre um poliedro é denominado problema de programação linear (abrevia-se PL). Usualmente descreve-se um PL na forma:

$$
\left\{\begin{array}{l}
\operatorname{maximize} c^{T} x \\
\text { sujeito a } A x \leq b
\end{array} \text { ou } \max \left\{c^{T} x: A x \leq b\right\}\right.
$$

sendo $A \in R^{m \times n}, b \in \mathbb{R}^{m}$ e $c \in \mathbb{R}^{n}$.

Um vetor $\tilde{x} \in \mathbb{R}^{n}$ tal que $A \tilde{x} \leq b$ é denominado uma solução viável do PL. Se $\tilde{x}$ é uma solução viável tal que $c^{T} \tilde{x} \geq c^{T} x^{\prime}$ para toda solução viável $x^{\prime}$ do PL, entào $\hat{x}$ é denominada uma solução ótima. A função $c^{T} x$ é comumente referida como função objetivo.

Um PL com restrição de integridade é chamado problema de programação linear inteira e é denotado por PLI. São problemas da seguinte forma:

$$
\left\{\begin{array}{ll}
\operatorname{maximize} & c^{T} x \\
\text { sujeito a } & A x \leq b \\
& x \text { inteiro }
\end{array} \quad \text { ou } \max \left\{c^{T} x: A x \leq b, \quad x \text { inteiro }\right\}\right.
$$

O problema de programação linear obtido de um PLI, ignorando a restrição " $x$ é inteiro", é chamado problema (de programação) linear relaxado. 
Os problemas pesquisados nesta tese podem ser formulados como PLI com a restrição especial de que $x \in\{0,1\}^{n}$. Freqüentemente, problemas de otimização combinatória podem ser formulados como PLI. Infelizmente, uma tal formulação não é muito útil do ponto de vista algorítmico, já que o problema PLI é $\mathbb{N} \mathbb{P}$-difícil (Garey e Johnson [1979]).

Resultados fundamentais e outros conceitos de programação linear e programação linear inteira podem ser encontrados em Chvátal [1983], Dantzig [1963] e Schrijver [1986]. Para complementar a teoria dos poliedros recomendamos Grünbaum [1967], Schrijver [1986], Pulleyblank [1983] e Br $\phi$ ndsted [1983].

\section{I.3 Teoria da complexidade computacional}

Nesta secção, discutimos de uma maneira informal e intuitiva alguns conceitos básicos da teoria da complexidade computacional. Definições precisas destes conceitos e resultados nesta teoria podem ser encontrados em Garey e Johnson [1979] ou em Aho, Hopcroft e Ullman [1974].

Um problema é definido como uma questão com vários parâmetros ou variáveis cujos valores não são especificados e cuja solução deve satisfazer algumas propriedades. Se todos os parâmetros assumem valores específicos no problema, temos uma instância do problema. Por exemplo, a questão "existe num dado grafo $G$ um $k$-circuito?" é um problema com parâmetros $G$ e $k$, em aberto. Se especificarmos o grafo $G$ e o valor de $k$, temos uma instância do problema.

Informalmente, um algoritmo para um determinado problema é um procedimento finito, executado passo a passo que, aplicado a cada instância desse problema, produz uma solução. Na discussão de algoritmos, emerge naturalmente a seguinte questão: "como medir a eficiência de um algoritmo?" Para se determinar a eficiência de um algoritmo, deve-se considerar os esforços computacionais exigidos por ele e por esta razão várias medidas de complexidade computacional foram sugeridas por diferentes pesquisadores. 
Em particular, poderíamos mencionar os trabalhos de Cook [1971] e Karp [1972]. Como o tempo de computação é a medida mais utilizada para se determinar a eficiência ou a complexidade de um algoritmo, nos restringimos a ele como a medida de complexidade de nossos algoritmos.

É intuitivo perceber que o tempo que um algoritmo gasta para resolver uma instância de um problema varia de acordo com o tamanho da instância. A medida do tamanho de uma instância (referida como o tamanho da instância) é feita com o auxílio de um esquema de codificação, isto é, uma função que mapeia cada instância do problema em uma cadeia finita de símbolos de um alfabeto fixo. O tamanho de uma instância $I$, que será denotado por $\mathbf{C}(\mathbf{I})$ é referido como a entrada da instância ao computador. Naturalmente, $C(I)$ depende do esquema de codificação adotado e do modelo de computador que executará o algoritmo. Para nossa discussão informal, podemos adotar por exemplo, código binário e máquina de Turing ou números codificados em uma base fixa diferente da unidade e um computador real qualquer.

Dado um algoritmo $A$ para um problema $P$, a complexidade de tempo de $A$ é uma função $f_{A}$ que mapeia cada natural $n$ no tempo máximo requerido por $A$ para a solução de uma instância $I$ de $P$ tal que $C(I) \leq n$. Um algoritmo $A$ é polinomial se existe um polinômio $p: \mathbb{N} \rightarrow \mathbb{N}$ tal que $f_{A}(n) \leq c|p(n)|$ para uma constante $c$. Neste caso, dizemos que a função $f_{A}(n)$ é da ordem de $p(n)$ e a denotamos por $f_{A}(n)=O(p(n))$. Um algoritmo polinomial é usualmente aceito como sendo um algoritmo eficiente e um problema é geralmente considerado fácil se existe um algoritmo polinomial que o resolva.

A seguir, veremos uma classificação sistemática dos problemas, de acordo com a complexidade computacional de seus algoritmos.

Vamos analisar dois tipos de problemas: os chamados problemas de decisão que admitem, para cada instância, apenas um dos dois tipos de solução: "SIM" ou "NÃO", e os problemas de busca, que admitem, para cada instância, um número arbitrário de 
soluções. Por exemplo, "existe num dado grafo $G$ um circuito" é um problema de decisão e "encontre em $G$ um circuito" é um problema de busca.

Inicialmente, consideremos apenas problemas de decisão e denotemos por IP a classe dos problemas de decisão que podem ser resolvidos por um algoritmo polinomial. Entre os problemas mais famosos para os quais não se conhece algoritmos polinomiais para resolvê-los, destaca-se o problema do circuito hamiltoniano, enunciado como: "dado um grafo $G, G$ é hamiltoniano?" Observe que para cada instância deste problema, cuja resposta é SIM, é possível, dado um circuito, verificar através de um algoritmo polinomial se ele é um circuito hamiltoniano. Problemas com esta propriedade pertencem à classe dos problemas referida como INIP.

Informalmente, define-se a classe INIP em termos de um algoritmo não determinístico, isto é, a classe dos problemas de decisão $Q$, tais que para cada instância $I$ de $Q$ cuja resposta é SIM, existe uma estrutura $S$ através da qual, pode-se verificar a veracidade da resposta em tempo polinomial em $C(I)$. Observe que não precisamos saber como encontrar $S$ em tempo polinomial, apenas verificar a resposta em tempo polinomial, utilizando a estrutura $S$.

Dizemos que um problema $Q$ pertence à classe co - INIP se a negação de $Q$ é um problema em INIP. A té o momento, não se sabe se o problema de decidir se um grafo é não hamiltoniano está em INIP. Portanto, encontra-se em aberto a questão de decidir se o problema do circuito hamiltoniano pertence à classe $\mathbb{N} I P \cap c o-I N I P$.

É fácil ver que $\mathbb{P} \subseteq \mathbb{N} \mathbb{P}$. A implicação contrária tem sido vastamente investigada, desde 1971 (Cook [1971], Karp [1972]) e é até hoje um dos maiores problemas em aberto na área de teoria da computaçào. Entretanto, a maioria dos pesquisadores desta área acredita na conjectura de que $\mathbb{P} \neq \mathbb{N} \mathbb{P}$. Para esclarecer melhor essa conjectura, vamos definir uma subclasse especial de problemas em $\mathbb{N} \mathbb{P}$, a classe dos problemas $\mathbb{N} \mathbb{P}$-completos.

Dados dois problemas de decisão $Q$ e $Q^{\prime}$, dizemos que $Q$ é polinomialmente trans- 
formável em $Q^{\prime}$ (denotamos por $Q \sim Q^{\prime}$ ) se existe um algoritmo que a cada instância $I$ de $Q$, produz em tempo polinomial uma instância $I^{\prime}$ de $Q^{\prime}$ tal que a solução de $I$ é SIM se e somente se a solução de $I^{\prime}$ é SIM.

Um problema de decisão $Q$ é $\mathbb{N} \mathbb{P}$-completo se satisfaz às duas condições abaixo:

(i) $Q \in \mathbb{N} \mathbb{P}$;

(ii) $\forall Q^{\prime} \in \mathbb{N} \mathbb{P}, Q^{\prime} \sim Q$.

Da transitividade da relação $\sim$, segue que se $Q \in \mathbb{N} \mathbb{P}$ e existe um problema $\mathbb{N} \mathbb{P}$ completo $\hat{Q}$ tal que $\hat{Q} \sim Q$, então $Q$ é INIP-completo. Este é o método comumente usado para se demonstrar que um problema é INIP-completo. Um outro método para verificarmos que um problema $Q$ é $\mathbb{N} \mathbb{P}$-completo é o da restrição, que consiste simplesmente em mostrar que $Q$ contém um problema conhecido INIP-completo $Q^{\prime}$, como um caso especial. Observe que a prova de que um problema qualquer $Q$ pertence simultaneamente às classes $\mathbb{N I P}$-completo e $\mathbb{P}$ implica $\mathbb{P}=\mathbb{N P}$. Por este motivo, acredita-se que os problemas $\mathbb{N I P}$-completos são os mais difíceis de $\mathbb{N} \mathbb{P}$. O problema do circuito hamiltoniano é $\mathbb{N} \mathbb{P}$ completo e uma longa lista destes problemas podem ser encontradas em Garey e Johnson [1979].

Dizemos que um problema de busca $Q$ é Turing-redutível a um outro problema de busca $Q^{\prime}$ (denota-se por $Q \sim_{T} Q^{\prime}$ ) se:

i) existe um algoritmo $A$ que resolve $Q$ usando um algoritmo hipotético $S$ que resolve $Q^{\prime}$, como um procedimento.

ii) $A$ é um algoritmo polinomial para $Q$ se $S$ é um algoritmo polinomial para $Q^{\prime}$.

Um problema de busca $Q$ é $\mathbb{N} \mathbb{P}$-difícil se existe um problema de decisão $Q^{\prime} \mathbb{N} \mathbb{P}$ completo tal que $Q^{\prime} \sim_{T} Q$. 
É fácil verificar que a existência de um algoritmo polinomial para resolver um problema $\mathbb{N} \mathbb{P}$-difícil implica na igualdade $\mathbb{P}=\mathbb{N} \mathbb{P}$, ou seja, os problemas da classe $\mathbb{N} \mathbb{P}$-difícil são pelo menos tão difíceis quanto os da classe $\mathbb{N} \mathbb{P}$.

Da transitividade da relação $\sim_{T}$ segue que se um problema $Q$ é $\mathbb{N} I P$-difícil e $Q \sim_{T} Q^{\prime}$ então $Q^{\prime}$ é INIP-difícil.

Para finalizar esta seção, faremos algumas observações sobre complexidade computacional em problemas de otimização, que são os objetos principais da nossa pesquisa.

Sabe-se que a maioria dos problemas de otimização são $\mathbb{N} \mathbb{P}$-difíceis. Portanto, sob a hipótese de que $\mathbb{P} \neq \mathbb{N} \mathbb{P}$, é pouco provável que se encontre algoritmos polinomiais para resolvê-los. Logo, é razoável procurar heurísticas que produzem soluções aproximadas ou pesquisar instâncias especiais do problema que podem ser resolvidos por algoritmos polinomiais.

Mostramos agora como um problema de decisão pode ser derivado de um problema de otimização. Se o problema de otimização $P$ investiga a existência de uma estrutura de custo mínimo entre todas as estruturas de um certo tipo (por exemplo, um circuito hamiltoniano de custo mínimo em um grafo completo com custos nas arestas), podemos associá-lo com o problema de decisão $Q$ que inclui um limite $k$ como um parâmetro adicional e investigar a existencia de uma estrutura de custo no máximo igual a $k$ (ou no mínimo igual a $k$, se o problema de otimização for de maximização). Logo, $Q$ está relacionado com $P$ da seguinte forma: Se resolvemos $P$ eficientemente, podemos resolver $Q$ eficientemente. Mais precisamente, ocorre que $Q \sim_{T} P$. Portanto se $Q$ for $\mathbb{N P}$-completo, $P$ será $\mathbb{N} I P$-difícil. Este é o procedimento que usaremos no próximo capítulo, para provar que um problema de otimização é $\mathbb{N} \mathbb{P}$-difícil.

Para finalizar, cabe aqui mencionar os artigos de Edmonds [1965] e Cobham [1965], da década de 60, que contêm algumas idéias seminais sobre eficiência de algoritmos, que foram fundamentais para o desenvolvimento da teoria de complexidade computacional. 


\section{Capítulo II}

\section{Problemas da Pré-Ordem Completa, da Pré-Ordem e da Ordem Parcial}

Este capítulo contém demonstrações estabelecendo a complexidade dos problemas da ordem parcial, da pré-ordem e da pré-ordem completa.

Em 1986, num estudo sobre a complexidade computacional dos chamados problemas de agregação de relações binárias, Wakabayashi [1986] mostrou a equivalência destes problemas com certos problemas de otimizaçào sobre digrafos, cujas instâncias são digrafos completos com pesos nas arestas, todos de mesma paridade. Nesse estudo, vários problemas foram provados serem $\mathbb{N} \mathbb{P}$-difíceis. Fazendo uso de idéias encontradas nesse trabalho, apresentamos aqui, a prova da complexidade computacional dos problemas de nosso interesse, introduzindo algumas simplificaçôes, já que não temos nenhuma restrição quanto à paridade dos pesos das arestas.

Devido a sua forte relação com os nossos problemas, inclúmos também o estudo da complexidade do problema da ordem linear. Este problema, também referido na 
literatura como o problema do torneio transitivo foi estudado por Reinelt [1985].

\section{II.1 Os problemas}

Como estabelecemos no capítulo anterior, $D_{n}=\left(V_{n}, A_{n}\right)$ denota o digrafo completo com $n$ vértices. Uma função $p: A_{n} \rightarrow \mathbb{Z}$ será referida como uma função peso nas arestas do digrafo. Se $A \subseteq A_{n}$ e $p$ é uma função de $A_{n}$ em $\mathbb{Z}$, então peso de $\mathbf{A}$, denotado por $p(A)$, é a soma dos pesos das arestas de $A$, isto é,

$$
p(A)=\sum_{\alpha \in A} p(\alpha) .
$$

Cada um dos problemas que definimos a seguir, tem como parâmetros um digrafo completo $D_{n}=\left(V_{n}, A_{n}\right)$ e uma função peso $p: A_{n} \rightarrow \mathbb{Z}$.

Problema da Ordem Linear (OL): Encontrar em $D_{n}$ uma ordem linear $A \subseteq A_{n}$ de peso máximo.

Problema da Pré-Ordem Completa (POC): Encontrar em $D_{n}$ uma pré-ordem completa $A \subseteq A_{n}$ de peso máximo.

Problema da Pré-Ordem (PO): Encontrar em $D_{n}$ uma pré-ordem $A \subseteq A_{n}$ de peso máximo.

Problema da Ordem Parcial (OP): Encontrar em $D_{n}$ uma ordem parcial $A \subseteq A_{n}$ de peso máximo.

Observe que a propriedade transitiva é comum às relações de ordem procuradas. $\mathrm{Na}$ ordem linear, pesquisamos subdigrafos geradores acíclicos transitivos totais; na ordem parcial, subdigrafos acíclicos transitivos; na pré-ordem completa, subdigrafos geradores transitivos totais; e na pré-ordem, subdigrafos transitivos. O diagrama abaixo ilustra a 
relação dessas ordens em um digrafo completo.

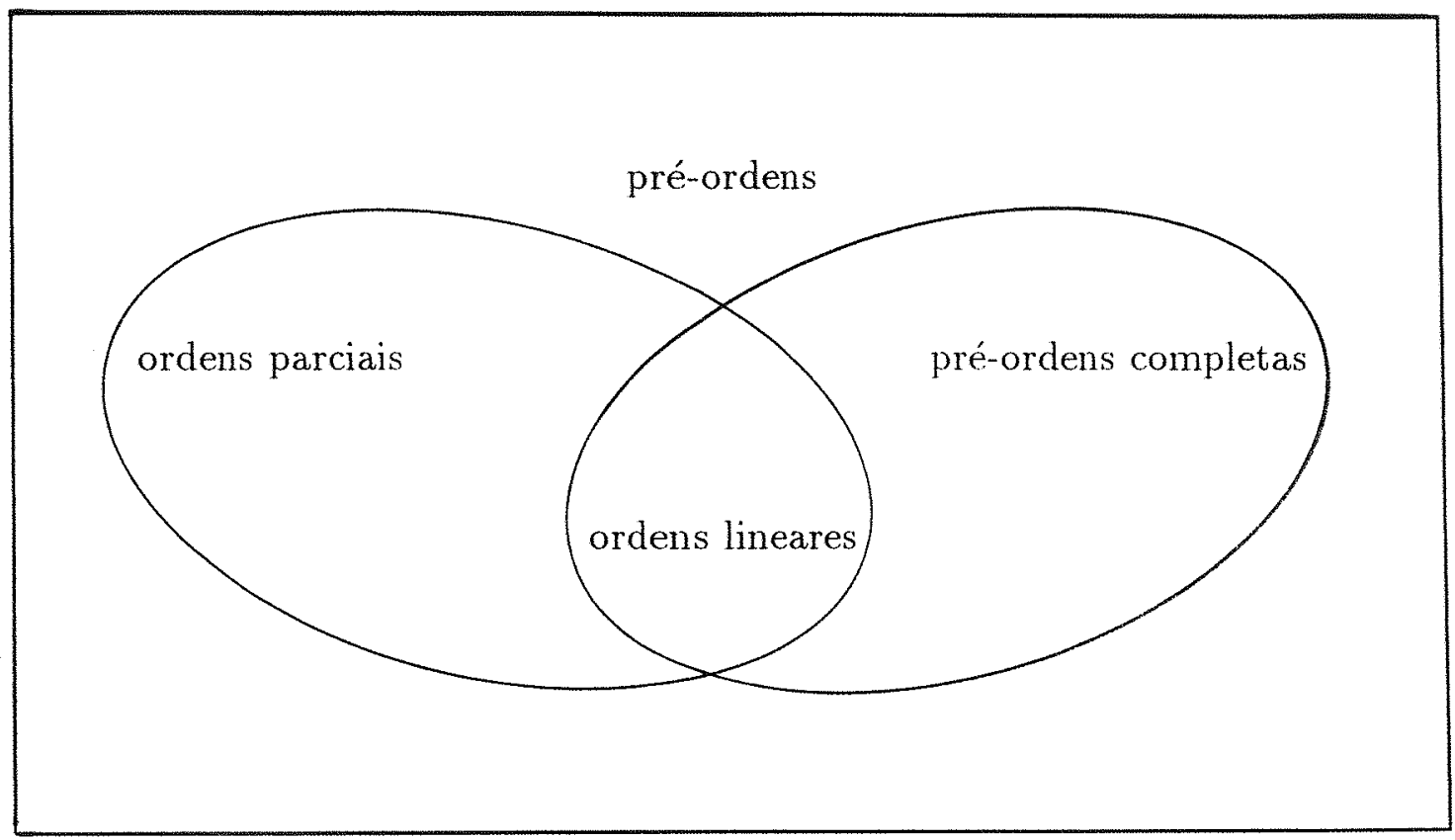

\section{II.2 Complexidade computacional}

Provaremos que os problemas $O L, O P, P O$ e $P O C$ são $\mathbb{N} \mathbb{P}$-difíceis, mostrando que o problema de decisão derivado de cada um destes problemas de otimização, conforme descrevemos no capítulo I, é INIP-completo. É imediato como resolver qualquer um destes problemas de decisão em tempo polinomial por um algoritmo não determinístico; assim, omitimos de nossas demonstrações o fato de que estes problemas pertencem à classe INIP. O problema do subdigrafo acíclico de peso máximo de um digrafo com peso nas arestas, será utilizado para mostrarmos que o problema de decisão associado a $O L$ é INIP-completo. Associados aos problemas de procura $O L, P O C, P O$ e $O P$, vamos definir os problemas 
de decisão $\widehat{O L}, \widehat{P O C}, \widehat{P O}$ e $\widehat{O P}$, respectivamente. Cada um destes problemas tem como parâmetros um digrafo completo $D_{n}=\left(V_{n}, A_{n}\right)$, uma função $p: A_{n} \rightarrow \mathbb{Z}$ e um inteiro $k$.

\section{Problemas:}

$\widehat{\mathrm{OL}}$ : Existe em $D_{n}$ uma ordem linear $B \subseteq A_{n}$, ou equivalentemente, um torneio transitivo $H=\left(V_{n}, B\right)$, tal que $p(B) \geq k$ ?

POC: Existe em $D_{n}$ uma pré-ordem completa $B \subseteq A_{n}$, ou equivalentemente, um subdigrafo transitivo total $H=\left(V_{n}, B\right)$, tal que $p(B) \geq k$ ?

$\widehat{\mathrm{PO}}$ : Existe em $D_{n}$ uma pré-ordem $B \subseteq A_{n}$, ou equivalentemente, um subdigrafo transitivo $H=\left(V_{n}, B\right)$, tal que $p(B) \geq k$ ?

$\widehat{\mathrm{OP}}$ : Existe em $D_{n}$ uma ordem parcial $B \subseteq A_{n}$, ou equivalentemente, um subdigrafo aciclico transitivo $H=\left(V_{n}, B\right)$, tal que $p(B) \geq k$ ?

O lema abaixo será usado para provarmos que os problemas $\widehat{O L}$ e $\widehat{O P}$ são $\mathbb{N} \mathbb{P}$ completos.

Lema II.1: Se $H=\left(V_{n}, B\right)$ é um subdigrafo acíclico de $D_{n}=\left(V_{n}, A_{n}\right)$, então $H$ é um subdigrafo de um torneio transitivo $H^{\prime}=\left(V_{n}, B^{\prime}\right)$ de $D_{n}$.

Prova: Indução em $n$.

Se $\left|V_{n}\right|=1$ ou $B=\emptyset$, o lema é obviamente válido. Suponha então que $\left|V_{n}\right|>1$ e $B \neq \emptyset$. É fácil ver que $H$ possui um sorvedouro, digamos $u$. Seja $\hat{H}:=H-u$. Pela hipótese de indução, $\hat{H}$ está contido em um torneio transitivo gerador de $D_{n}-u$, digamos $T$. Seja $H^{\prime}$ o digrafo obtido de $T$, adicionando a ele o vértice $u$ e o conjunto de arestas $\{(w, u): w \in V T\}$. É fácil ver que $H^{\prime}$ é um torneio transitivo gerador de $D_{n}$ que contém $H$. 
O teorema II.2 usa o fato de que o problema de decisão descrito abaixo é INIP-completo $(\operatorname{Karp}[1972])$.

\section{Problema do Subdigrafo Acíclico (PSA)}

Parâmetros: Digrafo $D=(V, A)$ sem laços; inteiro positivo $k$.

Questão: Existe $B \subseteq A$ tal que $D[B]$ é um subdigrafo acíclico e $|B| \geq k$ ?

Teorema II.2: O problema de decisão $\widehat{O L}$ é $\mathbb{N} \mathbb{P}$-completo.

Prova: Dada uma instância do PSA, que consiste de um digrafo $D=(V, A) \operatorname{com} n$ vértices e um inteiro positivo $k$, a correspondente instância do problema $\widehat{O L}$ será obtida da seguinte forma: $D_{n}=\left(V, A_{n}\right)$ é o digrafo completo obtido de $D$, adicionando arestas $(u, v)$ tais que $u \neq v$ e $(u, v) \notin A$, e os custos $p(\alpha)$ para cada $\alpha \in A_{n}$ são definidos como:

$$
p(\alpha):=\left\{\begin{array}{ll}
1, & \text { se } \alpha \in A \\
0, & \text { se } \alpha \in A_{n} \backslash A
\end{array} .\right.
$$

Vamos provar que $D$ tem um subdigrafo aciclico $H=(V, B) \operatorname{com}|B| \geq k$ se e somente se $D_{n}$ tem um torneio transitivo $H^{\prime}=\left(V, B^{\prime}\right) \operatorname{com} p\left(B^{\prime}\right) \geq k$.

(i) Seja $H=(V, B)$ um subdigrafo acíclico em $D$, tal que $|B| \geq k$. Então $H$ é um subdigrafo acíclico de $D_{n}$. Pelo lema II.1, existe um torneio transitivo $H^{\prime}=\left(V, B^{\prime}\right)$ em $D_{n}$ que contém $H$. Logo,

$$
p\left(B^{\prime}\right)=p(B)+p\left(B^{\prime} \backslash B\right) \geq|B| \geq k .
$$

(ii) Seja $H^{\prime}=\left(V, B^{\prime}\right)$ um torneio transitivo em $D_{n} \operatorname{com} p\left(B^{\prime}\right) \geq k$. Considere o conjunto $B:=\left\{\alpha \in B^{\prime}: p(\alpha)=1\right\}$. 
Como $p(\alpha)=0$ ou $p(\alpha)=1$ para todo $\alpha \in B^{\prime}$, segue que $|B|=p\left(B^{\prime}\right)$, donde $|B| \geq k$.

Então $H=(V, B)$ é um subdigrafo acíclico de $D, \operatorname{com}|B| \geq k$.

Observação: Segue da demonstração do teorema anterior, que $\widehat{O L}$ é $\mathbb{N} \mathbb{P}$-completo mesmo se $p(\alpha) \in\{0,1\}$ para toda aresta $\alpha$ e $k$ é um inteiro positivo. Vamos nos referir a este particular problema como $\widehat{O L}(0,1)$.

Teorema II.3: O problema de decisão $\widehat{O P}$ é INIP-completo.

Prova: Considere $D_{n}=\left(V_{n}, A_{n}\right)$ e $p: A_{n} \rightarrow \mathbb{Z}$ tal que $p(\alpha) \in\{0,1\}$ para todo $\alpha \in A_{n}$ e $k$ um inteiro positivo.

Pelo lema II.1, podemos afirmar que $D_{n}$ possui um subdigrafo aciclico transitivo (gerador) de peso pelo menos $k$ se e somente se $D_{n}$ possui um torneio transitivo gerador de peso pelo menos $k$, ou seja, se nos restringirmos às instâncias de $\widehat{O P}$ e $\widehat{O L}$ onde $p(\alpha) \in\{0,1\}$ e $k$ é um inteiro positivo, esses problemas são equivalentes. Como $\widehat{O L}(0,1)$ é $\mathbb{N} \mathbb{P}$-completo, seque que $\widehat{O P}$ é $\mathbb{N} \mathbb{P}$-completo.

Teorema II.4: O problema de decisão $\widehat{P O}$ é INIP-completo.

Prova: Mostraremos que $\widehat{O L}(0,1)$ é polinomialmente transformável em $\widehat{P O}$. Com este objetivo em mente, suponha que $D_{n}=\left(V_{n}, A_{n}\right)$, uma função $p$ e um inteiro positivo $k$ $\left(k \leq\left(\begin{array}{l}n \\ 2\end{array}\right)\right.$ ), são dados como uma instância de $\widehat{O L}(0,1)$ e vamos construir uma instância correspondente de $\widehat{P O}$, definida por $D_{n}^{\prime}, p^{\prime}$ e $k^{\prime}$. Assuma que $V_{n}=\{1,2, \ldots, n\}, n \geq 2$. Então $D_{n}^{\prime}=\left(V_{n}^{\prime}, A_{n}^{\prime}\right)$ é o digrafo completo de ordem $2 n$ tal que $V_{n}^{\prime}=\left\{u_{1}, u_{2}: u \in V_{n}\right\}$. Os pesos $p_{\alpha}^{\prime}$ das arestas $\alpha$ de $A_{n}^{\prime}$ são definidos como: 


$$
p_{\alpha}^{\prime}:=\left\{\begin{array}{cl}
-n^{8}, \text { se } \alpha=\left(u_{1}, u_{2}\right), \quad u \in V_{n} \\
n^{4}, \text { se } \alpha=\left(u_{2}, u_{1}\right), \quad u \in V_{n} \\
-n^{2} \quad, \text { se } \alpha=\left(u_{2}, v_{1}\right), \quad u \text { e } v \in V_{n}, \quad u \neq v \\
0 \quad, \text { se } \alpha=\left(u_{2}, v_{2}\right), \quad u \text { e } v \in V_{n}, \quad u \neq v \\
p_{u v}+n^{2} \quad, \text { se } \alpha=\left(u_{1}, v_{1}\right), \quad 1 \leq u<v \leq n \\
p_{v u}+n^{2}, \text { se } \alpha=\left(v_{1}, u_{2}\right), \quad 1 \leq u<v \leq n \\
n^{2}, \text { em caso contrário. }
\end{array}\right.
$$

O valor de $k^{\prime}$ é definido por:

$$
k^{\prime}:=k+n^{5}+\left(\begin{array}{l}
n \\
2
\end{array}\right) n^{2}
$$
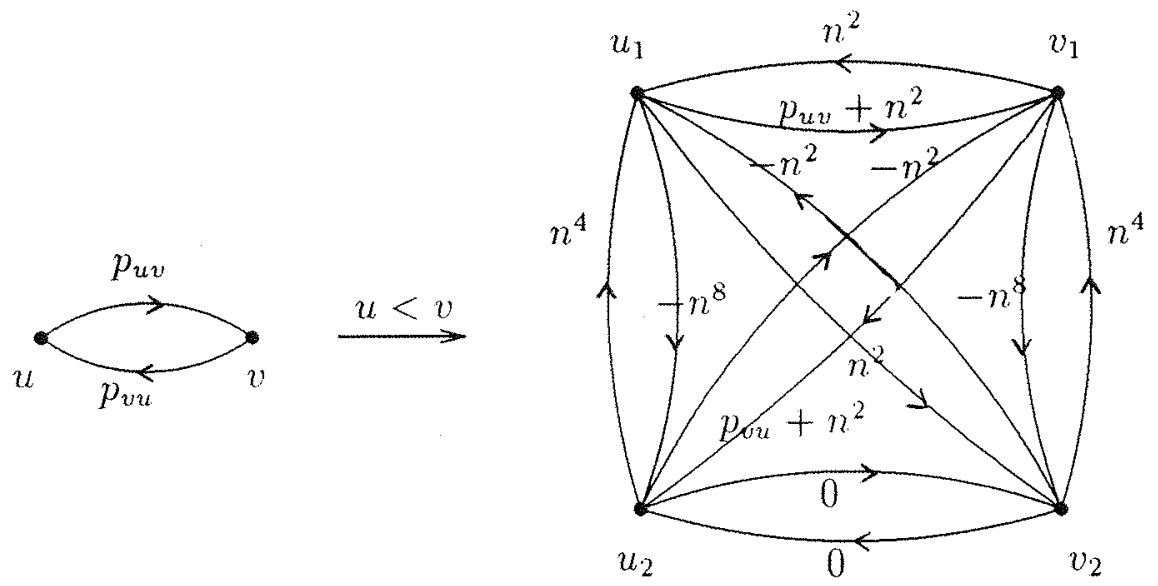

Figura II.1

Vamos provar que $D_{n}=\left(V_{n}, A_{n}\right)$ possui um torneio transitivo $H=\left(V_{n}, B\right) \mathrm{com}$ $p(B) \geq k$ se e somente se $D_{n}^{\prime}=\left(V_{n}^{\prime}, A_{n}^{\prime}\right)$ possui um subdigrafo transitivo $H^{\prime}=\left(V_{n}^{\prime}, B^{\prime}\right)$ $\operatorname{com} p^{\prime}\left(B^{\prime}\right) \geq k^{\prime}$.

Observe que para cada par $u, v, 1 \leq u<v \leq n$, as arestas $(u, v)$ e $(v, u)$ em $D_{n}$ correspondem às arestas $\left(u_{1}, v_{1}\right)$ e $\left(v_{1}, u_{2}\right)$ em $D_{n}^{\prime}$, respectivamente, e que os pesos destas 
arestas acrescidos de $n^{2}$ constituem os pesos das respectivas arestas em $D_{n}^{\prime}$ (veja a figura II.1).

O peso da aresta $\left(u_{1}, u_{2}\right), u \in V_{n}$ foi escolhido convenientemente, de tal forma que as arestas $\left(u_{1}, v_{1}\right)$ e $\left(v_{1}, u_{2}\right)$ não possam pertencer simultaneamente a um subdigrafo transitivo $H^{\prime}=\left(V_{n}^{\prime}, B^{\prime}\right)$ de $D_{n}^{\prime}$, com $p\left(B^{\prime}\right) \geq k^{\prime}$. Assim, as correspondentes arestas darão origem a um torneio em $D_{n}$.

Os pesos das demais arestas e o valor de $k^{\prime}$ foram escolhidos de tal forma que todo subdigrafo transitivo $H^{\prime}=\left(V_{n}^{\prime}, B^{\prime}\right)$ de $D_{n}^{\prime}$ com $p^{\prime}\left(B^{\prime}\right) \geq k^{\prime}$ é um torneio transitivo em $D_{n}^{\prime}$. Com isto, esta mesma prova será suficiente para provarmos que $\widehat{P O}$ e $\widehat{P O C}$ são NP-completos.

(i) Dado um torneio transitivo $H=\left(V_{n}, B\right)$ em $D_{n}$, com $p(B) \geq k$, vamos construir $H^{\prime}=\left(V_{n}^{\prime}, B^{\prime}\right)$, definindo:

$$
B^{\prime}:=\left\{\left(u_{2}, u_{1}\right): u \in V_{n}\right\} \cup\left\{\left(u_{1}, v_{1}\right),\left(u_{1}, v_{2}\right),\left(u_{2}, v_{1}\right),\left(u_{2}, v_{2}\right):(u, v) \in B\right\}
$$

Para cada par $\alpha=(u, v), 1 \leq u, v \leq n$, seja $R_{\alpha}$ o seguinte subdigrafo básico.

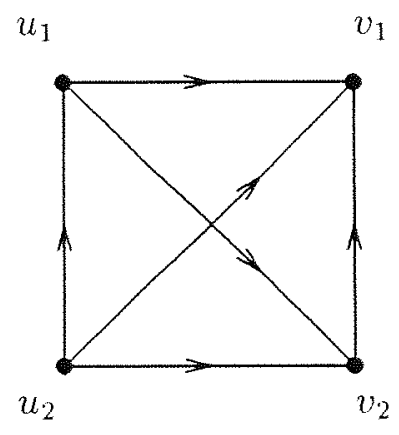


Vamos provar inicialmente que $p^{\prime}\left(B^{\prime}\right) \geq k^{\prime}$.

Note que $H^{\prime}$ é a união de todos os subdigrafos básicos $R_{\alpha}$, cada um deles correspondente à uma aresta $\alpha \in B$. Devido à estrutura bastante simétrica do grafo $H^{\prime}$, é fácil verificar que:

$$
\begin{aligned}
p^{\prime}\left(B^{\prime}\right) & =\sum_{(u, v) \in B} p^{\prime}\left(A R_{(u, v)}-\left(u_{2}, u_{1}\right)-\left(v_{2}, v_{1}\right)\right)+\sum_{u \in V_{n}} p^{\prime}\left(u_{2}, u_{1}\right) \\
& =\sum_{\alpha \in B}\left(p(\alpha)+n^{2}\right)+n n^{4} \\
& =p(B)+\left(\begin{array}{l}
n \\
2
\end{array}\right) n^{2}+n^{5} \geq k+n^{5}+\left(\begin{array}{l}
n \\
2
\end{array}\right) n^{2}=k^{\prime}
\end{aligned}
$$

Resta provar que $H^{\prime}$ é transitivo.

Claramente, cada subdigrafo básico de $H^{\prime}$ é transitivo. Vamos mostrar que se $e:=\left(u_{r}, v_{s}\right)$ e $f:=\left(v_{s}, w_{t}\right)$ com $r, s, t \in\{1,2\}$ são arestas de $B^{\prime}$ pertencentes a subdigrafos básicos distintos, então $g:=\left(u_{r}, w_{t}\right) \in B^{\prime}$. Note que $u, v$ e $w$ são dois a dois distintos, senão, e e $f$ estariam no mesmo subdigrafo básico.

Como $\left(u_{r}, v_{s}\right) \in B^{\prime}$ e $\left(v_{s}, w_{t}\right) \in B^{\prime}$, sendo $u, v$ e $w$ dois a dois distintos, da definição de $B^{\prime}$, segue que $(u, v)$ e $(v, w) \in B$. Como $H$ é transitivo, $(u, w) \in B$. Logo, $\left(u_{p}, v_{q}\right) \in B^{\prime}$ para $p, q \in\{1,2\}$, donde $g \in B^{\prime}$.

Então $H^{\prime}$ é um torneio transitivo com $p\left(B^{\prime}\right) \geq k^{\prime}$, como queríamos demonstrar.

(ii) Seja $H^{\prime}=\left(V_{n}^{\prime}, B^{\prime}\right)$ um subdigrafo transitivo de $D_{n}^{\prime} \operatorname{com} p^{\prime}\left(B^{\prime}\right) \geq k^{\prime}$. Antes de construirmos um torneio transitivo $H=\left(V_{n}, B\right)$ de $D_{n}, \operatorname{com} p(B) \geq k$, vamos verificar algumas propriedades do digrafo $H^{\prime}$. 
(P1) $B^{\prime}$ não contém nenhuma aresta $\alpha \operatorname{com} p_{\alpha}^{\prime}=-n^{8}$.

Suponha que $B^{\prime}$ contenha tal aresta. Então,

$$
\begin{aligned}
p^{\prime}\left(B^{\prime}\right) & \leq-n^{8}+n^{5}+\left(\begin{array}{l}
n \\
2
\end{array}\right)\left(2+4 n^{2}\right) \\
& =-n^{8}+n^{5}+n^{2}\left(\begin{array}{l}
n \\
2
\end{array}\right)+\left(2+3 n^{2}\right)\left(\begin{array}{l}
n \\
2
\end{array}\right) \\
& \leq-n^{8}+\frac{3 n^{4}}{2}+n^{5}+n^{2}\left(\begin{array}{l}
n \\
2
\end{array}\right) \\
& <n^{5}+n^{2}\left(\begin{array}{l}
n \\
2
\end{array}\right) \leq k^{\prime},
\end{aligned}
$$

uma contradição.

(P2) Para todo par $(u, v), 1 \leq u<v \leq n$, os pares de arestas $\left(u_{1}, v_{1}\right),\left(v_{1}, u_{2}\right)$ e $\left(v_{1}, u_{1}\right),\left(u_{1}, v_{2}\right)$ não pertencem simultaneamente ao grafo $H^{\prime}$.

Esta propriedade pode ser facilmente verificada a partir de (P1), dado que $H^{\prime}$ é transitivo.

(P3) $B^{\prime}$ contém todas as arestas $\alpha$ com $p_{c k}^{\prime}=n^{4}$.

Suponha que isto não ocorra. Dada a validade de (P1) e (P2), temos:

$$
\begin{aligned}
p^{\prime}\left(B^{\prime}\right) & \leq(n-1) n^{4}+\left(\begin{array}{l}
n \\
2
\end{array}\right)\left(2 n^{2}+1\right) \\
& =n^{5}+\left(\begin{array}{l}
n \\
2
\end{array}\right) n^{2}-n^{4}+\left(\begin{array}{l}
n \\
2
\end{array}\right)\left(n^{2}+1\right) \\
& <n^{5}+\left(\begin{array}{l}
n \\
2
\end{array}\right) n^{2} \\
& \leq k^{\prime} .
\end{aligned}
$$

Nos próximos dois casos, apresentaremos propriedades relativas às arestas de $B^{\prime}$ que têm ambos os extremos no conjunto $\left\{u_{1}, u_{2}, v_{1}, v_{2}\right\}$, sendo $u, v \in V_{n}$, $u \neq v$.

Com este objetivo, dados $u$ e $v, 1 \leq u \neq v \leq n$, seja

$$
\hat{B}=B^{\prime} \cap\left(A R_{\alpha} \cup A R_{\bar{\alpha}}\right), \quad \text { onde } \quad \alpha=(u, v) .
$$


(P4) $2 n^{4}+n^{2} \leq p^{\prime}(\hat{B}) \leq 2 n^{4}+n^{2}+1$.

Seja $X:=\left\{\left(u_{1}, v_{1}\right),\left(u_{1}, v_{2}\right),\left(v_{1}, u_{1}\right),\left(v_{1}, u_{2}\right)\right\} \quad$ e $\quad Y:=\left\{\left(u_{2}, v_{1}\right),\left(v_{2}, u_{1}\right)\right\}$. Entâo

$$
p^{\prime}(\hat{B})=2 n^{4}+p^{\prime}(\hat{B} \cap X)+p^{\prime}(\hat{B} \cap Y) .
$$

Se $\hat{B} \cap X=\emptyset$, então $p^{\prime}(\hat{B}) \leq 2 n^{4}$, donde $p^{\prime}(\hat{B}) \leq 2 n^{4}+n^{2}+1$.

Suponha agora que $\hat{B} \cap X \neq \emptyset$. Por (P2), temos que $|\hat{B} \cap X| \leq 2$, e portanto, $p^{\prime}(\hat{B} \cap X) \leq 2 n^{2}+1$. Por outro lado, de (P3) e da transitividade de $H^{\prime}$, vem que $|\hat{B} \cap Y| \geq 1$. Como as arestas de $Y$ têm peso $-n^{2}$, segue que

$$
p^{\prime}(\hat{B}) \leq 2 n^{4}+n^{2}+1
$$

Para completar a prova de (P4), suponha por contradiçào que $p^{\prime}(\hat{B})<2 n^{4}+n^{2}$. Neste caso, $p^{\prime}(\hat{B}) \leq 2 n^{4}+1 . \operatorname{Logo}$,

$$
\begin{aligned}
p^{\prime}\left(B^{\prime}\right) & =p^{\prime}(\hat{B})+p^{\prime}\left(B^{\prime} \backslash \hat{B}\right) \\
& \leq n^{5}+\left(\left(\begin{array}{l}
n \\
2
\end{array}\right)-1\right)\left(n^{2}+1\right)+1 \\
& =n^{5}+\left(\begin{array}{l}
n \\
2
\end{array}\right) n^{2}+\left(\begin{array}{l}
n \\
2
\end{array}\right)-n^{2}-1+1 \\
& <n^{5}+\left(\begin{array}{l}
n \\
2
\end{array}\right) n^{2} \\
& \leq k^{\prime}
\end{aligned}
$$

uma contradição.

(P5) Ou $\hat{B}=A R_{\text {c }}$ ou $\hat{B}=A R_{\bar{\kappa}}$.

Como $p^{\prime}(\hat{B}) \geq 2 n^{4}+n^{2}$, entâo $|X \cap \hat{B}| \geq 1$. Conforme vimos na demonstraçào de $(\mathrm{P} 4), \hat{B}$ possui uma aresta com peso $-n^{2}$. Como $n \geq 2$, segue que $|X \cap \hat{B}| \geq$ 2. De (P2), $|X \cap \hat{B}| \leq 2$, e portanto $|X \cap \hat{B}|=2$. 
Se $\left(u_{1}, v_{1}\right)$ e $\left(v_{1}, u_{1}\right) \in \hat{B}$, então $\left(u_{2}, v_{1}\right)$ e $\left(v_{2}, u_{1}\right) \in \hat{B}$, donde $p^{\prime}(\hat{B}) \leq 2 n^{4}+1$, uma contradição. Logo, $\left(u_{1}, v_{1}\right)$ e $\left(v_{1}, u_{1}\right)$ não pertencem simultaneamente a $\hat{B}$.

Suponha agora que $\left(u_{1}, v_{1}\right) \in \hat{B}$. Então $\left(v_{1}, u_{1}\right)$ e $\left(v_{1}, u_{2}\right) \notin \hat{B}$. Logo, $\left(u_{1}, v_{2}\right) \in$ $\hat{B}$. Da transitividade de $H^{\prime}$, segue que $\left(u_{2}, v_{2}\right) \in \hat{B}$ e $\left(u_{2}, v_{1}\right) \in \hat{B}$.

Se $\left(v_{2}, u_{1}\right) \in \hat{B}$, então $p^{\prime}(\hat{B}) \leq 2 n^{4}+1$, uma contradição. Se $\left(v_{2}, u_{2}\right) \in \hat{B}$, então $\left(v_{2}, u_{1}\right) \in \hat{B}$, uma contradição.

Logo, $\hat{B}=A R_{\alpha}$.

Com raciocínio análogo, verifica-se que se $\left(v_{1}, u_{1}\right) \in \hat{B}$, então $\hat{B}=A R_{\bar{\alpha}}$.

De (P5), segue que $H^{\prime}$ é um torneio em $D_{n}^{\prime}$. Agora estamos em condições de mostrar a existência de um torneio transitivo $H=\left(V_{n}, B\right)$ em $D_{n}$, com $p(B) \geq k$. Seja

$$
B:=\left\{(u, v):\left(u_{1}, v_{1}\right) \in B^{\prime}\right\}
$$

Para todo par $u, v, 1 \leq u, v \leq n$, segue de (P5) que $\left(u_{1}, v_{1}\right) \in B^{\prime}$, ou $\left(v_{1}, u_{1}\right) \in$ $B^{\prime}$, mas não ambos. $\operatorname{Logo},(u, v) \in B$, ou $(v, u) \in B$, mas não ambos, donde $H$ é um torneio.

Para provar que $H$ é transitivo, suponha que $(u, v)$ e $(v, w)$ são arestas de $B$, sendo $u \neq v, u \neq w$ e $v \neq w$.

Então $\left(u_{1}, v_{1}\right)$ e $\left(v_{1}, w_{1}\right)$ são arestas de $B^{\prime}$. Como $H^{\prime}$ é transitivo, $\left(u_{1}, w_{1}\right) \in B^{\prime}$ e portanto $(u, w) \in B$.

A validade da inequação $p(B) \geq k$ pode ser facilmente concluída, dada a estrutura de $B^{\prime}$, caracterizada pela propriedade (P5) e o fato de que $p^{\prime}\left(B^{\prime}\right) \geq k^{\prime}$. Como a transformação apresentada é polinomial, segue que $\widehat{P O}$ é $\mathbb{N} \mathbb{P}$-completo. 
Da demonstração acima, concluímos que se $H^{\prime}=\left(V_{n}, B^{\prime}\right)$ é um subdigrafo transitivo de $D_{n}^{\prime} \operatorname{com} p^{\prime}\left(B^{\prime}\right) \geq k^{\prime}$, então $H^{\prime}$ é um torneio transitivo de $D_{n}^{\prime}$. Portanto, esta mesma prova também garante a validade do seguinte teorema:

Teorema II.5: O problema de decisão $\widehat{P O C}$ é $\mathbb{N P}$-completo. 


\section{Capítulo III}

\section{Politopo da pré-ordem completa, da pré-ordem e da ordem parcial: dimensão e estrutura facial}

\section{III.1 Introdução}

Estudamos neste capítulo a estrutura facial dos politopos associados aos problemas da pré-ordem completa, da pré-ordem e da ordem parcial.

Como mencionamos na introdução deste trabalho, tais resultados poderiam ser usados no desenvolvimento de algoritmos para esses problemas. De fato, são conhecidos na literatura, exemplos de problemas de otimização combinatória, onde o conhecimento parcial das facetas dos politopos a eles associados combinado com técnicas de programação linear, resultou em algoritmos que se mostraram bastante satisfatórios (sob o ponto de vista computacional) na resoluçăo de instâncias reais. Tal abordagem, conhecida como método dos planos-de-corte, teve seu impulso com o sucesso obtido para o problema 
do caixeiro viajante (Grötschel \& Padberg [1985], Padberg \& Grötschel [1985], Crowder e Padberg [1980]).

Além do problema do caixeiro viajante, outros onde esta abordagem forneceu bons resultados foram: o problema da ordem linear (Grötschel, Jünger e Reinelt [1984a, 1984b]), o problema da partição em cliques (Grötschel \& Wakabayashi [1989]), o problema do corte máximo (Barahona \& Maccione [1982]) e o problema da ordenação seqüencial (Ascheuer, Escudero, Grötschel \& Stoer [1990]).

$\mathrm{Na}$ próxima seção discutiremos informalmente a idéia básica dessa abordagem poliédrica.

\section{III.2 Definições e resultados básicos}

Esta seção introduz os politopos $\mathbf{P}_{\mathbf{P O C}}, \mathbf{P}_{\mathbf{P O}}$ e $\mathbf{P}_{\mathrm{OP}}$, que associaremos aos problemas da pré-ordem completa, da pré-ordem e da ordem parcial, respectivamente. Por simplicidade, nos referiremos a esses politopos como da pré-ordem completa, da pré-ordem e da ordem parcial, respectivamente.

Convencionaremos aqui que cada um desses problemas tem como parte de uma instância um digrafo completo $D_{n}=\left(V_{n}, A_{n}\right)$ com $n$ vértices, e denotaremos por $P_{P O C}(n)$, $P_{P O}(n)$ e $P_{O P}(n)$, os respectivos politopos.

Dado um digrafo completo, $D_{n}=\left(V_{n}, A_{n}\right)$, lembramos que se $A \subseteq A_{n}$, o vetor característico de $A, x^{A}=\left(x_{e}^{A}\right)_{e \in A_{n}}$, é definido como

$$
x_{e}^{A}=\left\{\begin{array}{lll}
1, & \text { se } & e \in A \\
0 & \text {, se } & e \in A_{n} \backslash A .
\end{array}\right.
$$

Note que $x^{A}$ pode ser visto como um vetor no espaço $\left|A_{n}\right|$-dimensional $\mathbb{R}^{A_{n}}$ (cujos vetores estão indexados por $A_{n}$ ). Assim, podemos definir politopos em $\mathbb{R}^{A_{n}}$ tomando-se o fecho convexo de certos vetores característicos. É este o procedimento que adotaremos para definir cada um dos politopos $P_{P O C}(n), P_{P O}(n)$ e $P_{O P}(n)$, todos eles em $\mathbb{R}^{A_{n}}$. 
Definição III.1: O politopo da pré-ordem completa (pré-ordem, ordem parcial) é o fecho convexo dos vetores característicos dos pré-ordens completas (pré-ordens, ordens parciais) de $D_{n}$. Isto é,

- $P_{P O C}(n)=\operatorname{conv}\left\{x^{A} \in\{0,1\}^{A_{n}}: A\right.$ é uma pré-ordem completa de $\left.D_{n}\right\}$,

- $P_{P O}(n)=\operatorname{conv}\left\{x^{A} \in\{0,1\}^{A_{n}}: A\right.$ é uma pré-ordem de $\left.D_{n}\right\}$,

- $P_{O P}(n)=\operatorname{conv}\left\{x^{A} \in\{0,1\}^{A_{n}}: A\right.$ é uma ordem parcial de $\left.D_{n}\right\}$.

Como veremos a seguir, esses politopos têm uma estreita relação com o politopo da ordem linear, que denotaremos por $P_{O L}(n)$, definido analogamente, i.é:

- $P_{O L}(n)=\operatorname{conv}\left\{x^{A} \in\{0,1\}^{A_{n}}: A\right.$ é uma ordem linear de $\left.D_{n}\right\}$.

Este último politopo foi estudado por Reinelt [1985].

Note que cada um dos politopos foi definido como o fecho convexo de um conjunto de vetores $0 / 1$. É um fato conhecido e fácil de provar, que tais politopos têm como vértices exatamente o conjunto dos vetores $0 / 1$ considerado. Portanto, cada um dos politopos têm como vértices as possiveis soluçôes do problema em questão.

Segue então que se $p: A_{n} \rightarrow \mathbb{Z}$ é a função peso, que é dada como parte de uma instância do problema da pré-ordem completa (respectivamente pré-ordem e ordem parcial), esses problemas podem ser formulados da seguinte maneira:

$$
\begin{gathered}
P O C(n) \quad \begin{cases}\text { maximize } p^{T} x \\
\text { sujeito a } x \in P_{P O C}(n)\end{cases} \\
P O(n) \quad \begin{cases}\text { maximize } p^{T} x \\
\text { sujeito a } x \in P_{P O}(n)\end{cases} \\
O P(n) \quad \begin{cases}\text { maximize } p^{T} x \\
\text { sujeito a } & x \in P_{O P}(n)\end{cases}
\end{gathered}
$$


Note que os problemas acima são problemas de programação linear. Assim, poderse-ia pensar em aplicar técnicas de programação linear para resolvê-los. Em princípio, para que isto fosse possível, seria necessário conhecer, ou saber gerar eficientemente, uma descrição completa do politopo em questão, digamos $P$. Isto é, seria preciso encontrar um sistema de inequações lineares $A x \leq b$ tal que

$$
P=\{x: A x \leq b\}
$$

Sabe-se que para qualquer politopo $P$, tal sistema existe (Weyl [1935]). Não se sabe, porém, de nenhum problema combinatório INIP-difícil para o qual uma tal descrição completa seja conhecida. Além disso, conforme resultados devidos a Papadimitriou [1984], sabe-se que se o problema é INIP-difícil, então é pouco provável que se encontre uma descrição completa que seja 'boa' (no sentido de que o conjunto das facetas do politopo em questão esteja em $\mathbb{N} \mathbb{P}$ ).

Neste ponto, devemos observar que, mesmo se conhecêssemos uma tal descrição, se o sistema contiver um número exponencial (em relação ao tamanho da instância) de inequações, certamente não seria viável listá-las todas e utilizar métodos convencionais de programação linear para se resolver o problema.

Para efeito de raciocínio, suponha que conhecemos o sistema $A x \leq b$ que descreve $P$ e que queremos resolver: $\max \left\{p^{T} x: x \in P\right\}$ (são conhecidos vários problemas polinomiais onde isto ocorre, como por exemplo, o problema do emparelhamento (Edmonds [1985])). Um procedimento que poderia então ser usado, é o seguinte:

1. Comece com uma descriçâo parcial de $P$; i.e., um sistema $(S): A^{\prime} x \leq b^{\prime}$, tal que

$$
P \subseteq\left\{x: A^{\prime} x \leq b^{\prime}\right\}
$$


2. Resolva o problema de programação linear $\max \left\{p^{T} x: x\right.$ satisfaz $\left.S\right\}$, encontrando uma solução ótima $\bar{x}$.

3. Teste se $\bar{x}$ é um vértice de $P$. Se isto ocorrer, então $\bar{x}$ é solução ótima do problema original, pare. Senão, procure em $A x \leq b$ uma inequação $a^{T} x \leq \beta$ tal que $a^{T} \bar{x}>$ $\beta$. (Este último problema é chamado problema da separação ou identificação de inequaçôes violadas.)

Se foi encontrada uma tal inequação, chame de $S$ o sistema obtido de $S$ acrescentando-se essa inequação. Volte ao passo 2.

Se não foi encontrada, então pare. O ponto $\bar{x}$ não é solução ótima do problema original, mas fornece uma cota superior para o valor de $\max \left\{p^{T} x: x \in P\right\}$.

Note que, no procedimento acima descrito, parte-se de um subsistema de $A x \leq b$, i.e., de uma descrição parcial de. $P$, e vai-se gerando inequações violadas (pela solução corrente) à medida que isso se faz necessário. Eventualmente, sem fazer uso do sistema completo $A x \leq b$, é possível encontrar uma solução ótima da particular instância considerada.

O procedimento descrito é essencialmente o método dos planos-de-corte. Observe que este procedimento pode ser aplicado mesmo que não se conheça o sistema $A x \leq b$ que descreve $P$. Neste caso, ao invés de um tal sistema, usa-se um subsistema que dá apenas uma descrição parcial de $P$.

É importante notar que, para que este método seja útil do ponto de vista computacional, é preciso que o problema da separação seja resolvido eficientemente. É claro então que, se o sistema considerado contém um número exponencial de inequações, a identificação de inequações violadas não poderá ser feita através de um teste exaustivo. A este respeito, lembramos que se o problema da separação puder ser resolvido em tempo polinomial (no tamanho da instância) então o problema de otimização (relativo ao 
sistema considerado) pode ser resolvido em tempo polinomial (cf. Grötschel, Lovász \& Schrijver [1981]). No caso de problemas $\mathbb{N} \mathbb{P}$-difíceis, são usadas heurísticas para resolver o problema da separação.

Eventualmente, se no passo 3 não conseguimos resolver o problema da separação, então devemos aplicar o método 'branch-and-bound' para se chegar a uma solução ótima.

$\mathrm{Na}$ descrição informal acima, apresentamos apenas a essência do método dos planosde-corte. Recomendamos ao leitor interessado em detalhes e aspectos relativos à implementação desse método que consulte Padberg \& Grötschel [1985].

Finalizamos esta discussão introdutória, mostrando que os politopos $P_{P O C}(n), P_{P O}(n)$ e $P_{O P}(n)$ têm dimensão completa. Nas demonstrações deste fato e em outras a seguir, o conceito de inequação induzida por um digrafo será usada.

Se $G=(V, A)$ é um subdigrafo de $D_{n}=\left(V_{n}, A_{n}\right)$, dizemos que uma inequação da forma

$$
\sum_{\alpha \in A} a_{\alpha} x_{\alpha} \leq a_{0}, \quad a_{\alpha} \neq 0
$$

é uma inequação induzida por G. Para relacioná-la com um dos politopos $P_{O L}(n)$, $P_{O P}(n), P_{P O}(n)$ ou $P_{P O C}(n)$, basta estendê-la para uma inequação em $\mathbb{R}^{A_{n}}$, da seguinte forma:

$$
a^{T} x=\sum_{\alpha \in A_{n}} a_{\alpha x} x_{\alpha} \leq a_{0}, \text { onde } a \in \mathbb{R}^{A_{n}} \text { e } a_{\beta}=0 \text { se } \beta \in A_{n} \backslash A .
$$

Sempre que nos referimos a uma inequação, digamos $a^{T} x \leq a_{0}$, fica subentendido que ela é uma inequação induzida por um subdigrafo de $D_{n}$.

Proposição III.2: Os politopos $P_{P O}(n) \in P_{O P}(n)$ têm dimensão completa, isto é,

$$
\operatorname{dim} P_{P O}(n)=\operatorname{dim} P_{O P}(n)=\left|A_{n}\right|=n(n-1) .
$$


Prova: Basta observar que o vetor 0 e os vetores unitários em $\mathbb{R}^{A_{n}}$ são vetores de incidência de ordens parciais de $D_{n}$ e que este conjunto de vetores é independente afim.

Proposição III.3: O politopo PPoC $(n)$ tem dimensão completa, isto é,

$$
\operatorname{dim} P_{P O C}(n)=n(n-1)
$$

Prova: Considere $a^{T} x=b$ uma equação tal que

$$
P_{P O C}(n) \subseteq\left\{x \in\{0,1\}^{A_{n}}: a^{T} x=b\right\}
$$

Seja $\alpha=(u, v)$ uma aresta qualquer de $D_{n}$. Considere a ordem linear $O=\left\langle v, u, w_{1}, \ldots, w_{s}\right\rangle$ de $D_{n}$, sendo $\left\langle w_{1}, \ldots, w_{s}\right\rangle$ uma ordem linear arbitrária de $D_{n}-\{u, v\}$. Considerando o conjunto de arestas $E=A(O)$, observa-se que $E$ e $E \cup\{\alpha\}$ são pré-ordens completas de $D_{n}$. Então,

$$
\sum_{\beta \in E} a_{\beta} x_{\beta}=\sum_{\beta \in E} a_{\beta} x_{\beta}+a_{\alpha} x_{\alpha}=b, \quad \text { donde } a_{\alpha}=0
$$

Logo, a é um vetor de zeros e a prova está completa, de acordo com o Teorema I.3.

\section{III.3 Facetas}

Nesta seção apresentaremos várias classes de facetas para os politopos $P_{O P}(n), P_{P O}(n)$ e PPOC $(n)$. Algumas classes são comuns aos três politopos em estudo, outras a somente dois deles. Também descrevemos classes de facetas apenas para $P_{P O C}(n)$ ou para $P_{O P}(n)$.

O lema da elevação, demonstrado abaixo, será de grande utilidade nesta seção, pois dada uma inequação $a^{T} x \leq b$, a prova de que ela é uma faceta de um dos politopos, digamos de $P_{P O}(n)$, seguirá, na maioria das vezes, o seguinte roteiro: 
(i) mostra-se que $a^{T} x \leq b$ é uma inequação válida para $P_{P O}(n)$;

(ii) mostra-se que $a^{T} x \leq b$ é uma faceta de $P_{P O}(2)$ ou $P_{P O}(3)$;

(iii) mostra-se, usando o lema da elevação, que $a^{T} x \leq b$ é uma faceta de $P_{P O}(k)$, para todo $k, k \geq 2$ ou $k \geq 3$.

Juntamos em um único lema, resultados para os politopos $P_{P O}(k), P_{O P}(k)$ ou $P_{P O C}(k)$. Basta substituir todas as ocorrências de $P(k)$, que ocorrem no lema de elevação, e teremos um resultado para cada um dos politopos em questão.

No que segue, vamos supor que o digrafo completo $D_{n}=\left(V_{n}, A_{n}\right)$ é tal que $V_{n}=$ $\{1,2, \ldots, n\}$.

Lema III.4 (Lema da Elevação): Seja $a^{T} x \leq a_{0}$ uma inequação-faceta de $P(n), n \geq 2$. Defina $\hat{a} \in \mathbb{R}^{n(n+1)}$ como:

$$
\begin{aligned}
\hat{a}_{i, j} & =a_{i, j}, \text { se }(i, j) \in A_{n} \\
\hat{a}_{i, n+1} & =\hat{a}_{n+1, i}=0, \text { se } i=1, \ldots, n .
\end{aligned}
$$

Se $A \subseteq A_{n}$ é uma ordem linear de $D_{n}$ tal que $a^{T} x^{A}=a_{0}$, então $\hat{a}^{T} x \leq a_{0}$ é uma inequação-faceta de $P(n+1)$.

Prova: A inequação $\hat{a}^{T} x \leq a_{0}$ é obviamente válida para $P(n+1)$. Seja $F_{\hat{a}}$ a face de $P(n+1)$ definida por:

$$
F_{\hat{a}}=\left\{x \in P(n+1): \hat{a}^{T} x=a_{0}\right\} .
$$

Suponha que $\hat{b}^{T} x \leq b_{0}$ é uma inequação-faceta de $P(n+1)$ tal que

$$
F_{\hat{a}} \subseteq F_{\hat{b}}=\left\{x \in P(n+1): \hat{b}^{T} x=b_{0}\right\}
$$


Seja $O=<i_{1}, i_{2}, \ldots, i_{n}>$ uma ordem linear de $D_{n}$ tal que $a^{T} x^{A(O)}=a_{0}$. Vamos construir, para cada $j \in\{1, \ldots, n\}$, as seguintes ordens lineares de $D_{n+1}$ :

$$
\begin{aligned}
& O_{1}^{j}=\left\langle i_{1}, \ldots, i_{j}, n+1, i_{j+1}, \ldots, i_{n}\right\rangle \quad \mathrm{e} \\
& O_{2}^{j}=\left\langle i_{1}, \ldots, i_{j-1}, n+1, i_{j}, \ldots, i_{n}\right\rangle .
\end{aligned}
$$

Considere agora os conjuntos de arestas:

$$
A_{i}^{j}=A\left(O_{i}^{j}\right), \quad i=1,2
$$

Como $a^{T} x^{A(O)}=a_{0}$ e os conjuntos $A_{i}^{j}(1 \leq i \leq 2,1 \leq j \leq n)$ foram obtidos de $A(O)$, adicionando arestas tais que um de seus extremos é o vértice $n+1$, segue que,

$$
\hat{a}^{T} x^{A_{1}^{3}}=\hat{a}^{T} x^{A_{2}^{3}}=a_{0}, \quad j \in\{1, \ldots, n\} .
$$

Lembrando que $F_{\hat{a}} \subseteq F_{\hat{b}}$, concluímos que

$$
\hat{b}^{T} x^{A_{1}^{3}}=\hat{b}^{T} x^{A_{2}^{j}}=b_{0}, \quad j \in\{1, \ldots, n\} .
$$

Logo, como $A_{1}^{j} \Delta A_{2}^{j}=\left\{\left(i_{j}, n+1\right),\left(n+1, i_{j}\right)\right\}$, temos que

$$
\hat{b}_{n+1, i_{j}}=\hat{b}_{i_{j, n+1}} \text { para todo } j \in\{1, \ldots, n\} .
$$

Vamos demonstrar agora que $\hat{b}_{n+1, i}=0$ para $j \in\{1, \ldots, n\}$. Inicialmente, suponha que $P(n)=P_{P O}(n)$ ou $P(n)=P_{P O C}(n)$. Suponha que $j \in\{1, \ldots, n\}$ e considere o seguinte conjunto de arestas:

$$
A_{3}^{j}=A_{1}^{j} \cup\left\{\left(n+1, i_{j}\right)\right\}
$$

Observe que $A_{1}^{j}$ e $A_{3}^{j}$ são pré-ordens completas de $D_{n+1}$ que satisfazem

$$
\hat{a}^{T} x^{A_{1}^{j}}=\hat{a}^{T} x^{A_{3}^{j}}=a_{0} .
$$

De $F_{\hat{a}} \subseteq F_{\hat{b}}$, vem que $\hat{b}^{T} x^{A_{1}^{3}}=\hat{b}^{T} x^{A_{3}^{3}}=b_{0}$, donde

$$
\hat{b}_{n+1, i_{j}}=0
$$


Suponha agora que $P(n)=P_{O P}(n)$. Neste caso, basta fixar $j \in\{1, \ldots, n\}$ e considerar as ordens parciais $A_{1}^{j}$ e $A_{4}^{j}$ de $D_{n+1}$, sendo:

$$
A_{4}^{j}=A_{1}^{j} \backslash\left\{\left(i_{j}, n+1\right)\right\}
$$

Com raciocínio análogo, concluímos que $\hat{b}_{i_{j}, n+1}=0$. Portanto,

$$
\hat{b}_{i j, n+1}=0 \quad \text { para todo } j \in\{1, \ldots, n\} \text {. }
$$

De (2) e (3) ou (2) e (4), segue que $\hat{b}_{i j}=0$ para toda aresta $(i, j) \in A_{n+1}$, tal que $i=n+1$ ou $j=n+1$. Logo, $b^{T} x \leq b_{0}$ é válida para $P(n)$, sendo $b$ a restrição de $\hat{b}$ às arestas de $A_{n}$.

De (1), segue que,

$$
\left\{x \in P(n): a^{T} x=a_{0}\right\} \subseteq\left\{x \in P(n): b^{T} x=b_{0}\right\} .
$$

Como $a^{T} x \leq a_{0}$ é faceta de $P(n)$ e cada politopo tem dimensão completa, segue que $b^{T} x \leq b_{0}$ é uma faceta de $P(n)$. Logo, pelo teorema I.6, existe $\mu$ real, $\mu \geq 0$ tal que

$$
a^{T}=\mu b^{T} .
$$

De $\hat{a}_{i, n+1}=\hat{a}_{n+1, i}=\hat{b}_{i, n+1}=\hat{b}_{n+1, i}=0$ para $i \in\{1, \ldots, n\}$ e da equação (5), concluímos que $\hat{a}^{T}=\mu \hat{b}^{T}$. Portanto, $\hat{a}^{T} x \leq a_{0}$ é uma faceta de $P(n+1)$, como queríamos demonstrar.

Apresentamos a seguir várias classes de inequações-faceta para os três politopos em estudo. A figura abaixo relaciona cada classe de faceta com seus respectivos politopos.

Embora a figura sugira a existência de inequaçóes-facetas apenas do politopo $P_{P O}(n)$, não conseguimos encontrar nenhuma classe de inequaçôes que confirme este fato. Como também não provamos que toda faceta de $P_{P O}(n)$ é faceta de $P_{P O C}(n)$ ou de $P_{O P}(n)$, eventualmente a relação de inclusão entre estas classes pode ser diferente. 
Facetas de $P_{P O C}(n)$

Facetas de $P_{P O}(n)$

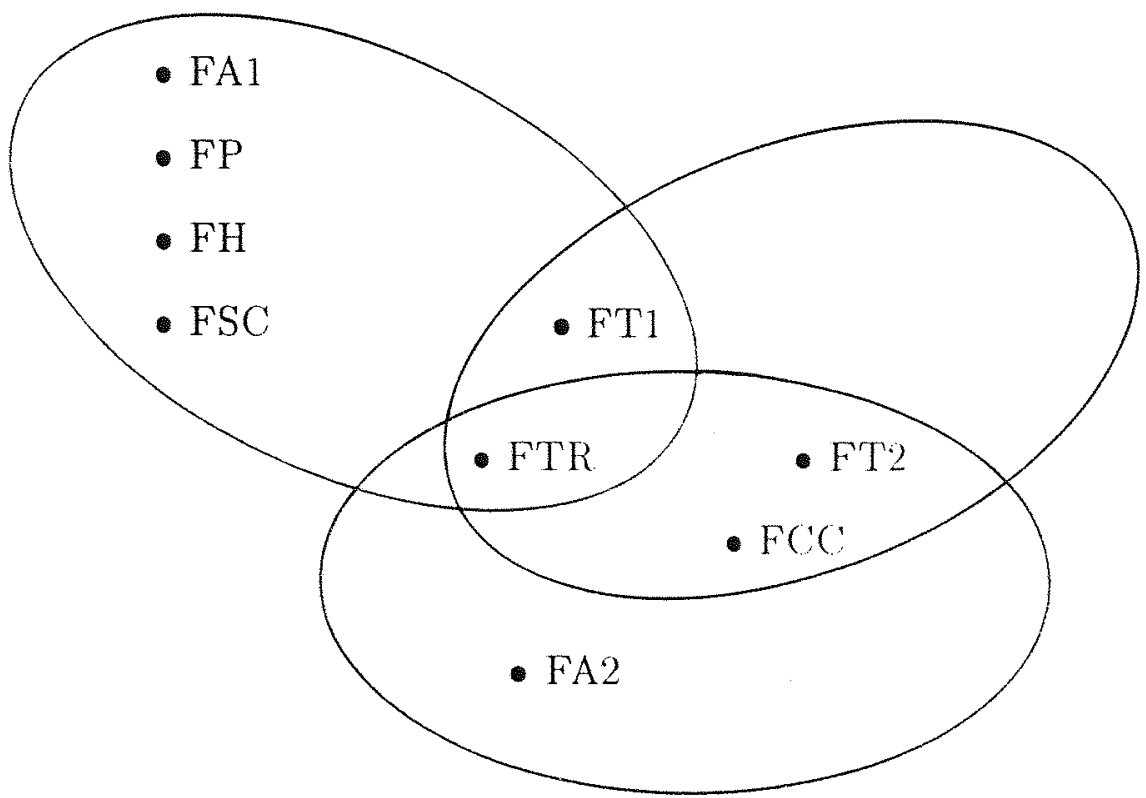

Facetas de $P_{O P}(n)$

- FTR. Facetas dos triângulos

$$
x_{i, j}+x_{j, k}-x_{i, k} \leq 1, \quad n \geq 3
$$

- FA. Facetas dos arcos paralelos

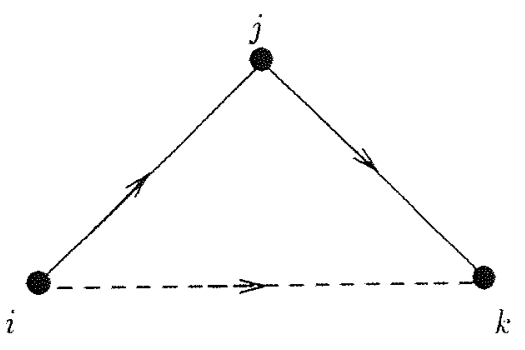

FA1. $x_{i, j}+x_{j, i} \geq 1$

FA2. $x_{i, j}+x_{j, i} \leq 1$

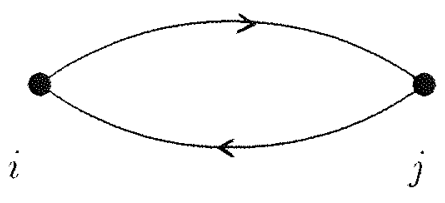


- FT. Facetas triviais

FT1. $x_{i, j} \leq 1$

FT2. $x_{i, j} \geq 0$

- FCC. Facetas dos circuitos ímpares com 2-cordas

$$
x(C)-x(C O) \leq\left\lfloor\frac{|C|}{2}\right\rfloor
$$

induzida por um digrafo $G$, sendo $A G=C \cup C O$, onde $C$ é o conjunto das arestas de um circuito ímpar dirigido e $C O$ é o conjunto das 2-cordas de $C,|C| \geq 5$.

- FP. Facetas dos pentágonos

$$
3 x(C)-3 x(C O)+x(\bar{C})-x(\overline{C O}) \leq 4,
$$

induzida por $D_{5}$, sendo $C$ o conjunto das arestas de um circuito hamiltoniano em $D_{5}, C O$ o conjunto das 2-cordas de $C, \bar{C}$ o reverso de $C$ e $\overline{C O}$ o reverso de $C O$.

- FH. Facetas dos heptágonos

$$
4 x(C)-4 x(C O)+2 x(\bar{C})-2 x(\overline{C O})+x(D)-x(\bar{D}) \leq 9,
$$

induzida por $D_{7}$, sendo $C$ as arestas de um circuito hamiltoniano de $D_{7}, C O$ o conjunto das 2-cordas de $C$ e $D$ o conjunto das 3-cordas de $C$. 
- FSC. Facetas das semi-k-cercas, $k \geq 3$

$$
x\left(A^{+}\right)-x\left(A^{-}\right) \leq k^{2}-2 k+2,
$$

induzida por um digrafo bipartido $G=\left(S \cup I, A^{+} \cup A^{-}\right)$, sendo $S=\left\{v_{1}, \ldots, v_{k}\right\}, I=$ $\left\{u_{1}, \ldots, u_{k}\right\}, A^{-}=\left\{\left(u_{i}, v_{i}\right): 1 \leq i \leq k-1\right\}$ e $A^{+}=\left\{\left(v_{k}, u_{k}\right)\right\} \cup\left\{\left(u_{i}, v_{j}\right): 1 \leq i, j \leq\right.$ $k$ e $i \neq j\}$.

\section{III.3.1 Facetas dos triângulos}

As inequações comuns aos três politopos, que surgem naturalmente de suas definições, estão relacionadas com a propriedade transitiva. Considere a seguinte definição:

Definição III.5: Seja $G$ um subdigrafo de $D_{3}, \operatorname{com} V G=\{i, j, k\}$ e $A G=\{\alpha, \beta, \gamma\}$, sendo $\alpha=(i, j), \beta=(j, k)$ e $\gamma=(i, k)$. Então a inequaçào induzida por $G$,

$$
x_{\alpha}+x_{\beta}-x_{\gamma} \leq 1
$$

é denominada inequaçâo-triângulo.

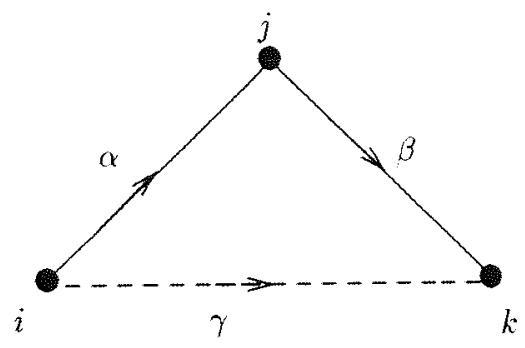

Observe que $D_{3}$ possui seis subdigrafos que induzem inequaçòes-triângulo duas a duas distintas.

Teorema III.6: Seja $G$ um subdigrafo de $D_{3}$, conforme a definiçâa anterior. Entâo a inequaçâo-triângulo induzida por $G$,

$$
x_{\alpha}+x_{\beta}-x_{\gamma} \leq 1
$$

é uma faceta de $P_{O P}(n), P_{P O}(n) \in P_{P O C}(n), n \geq 3$. 
Prova: A validade da inequação-triângulo para $P_{O P}(n), P_{P O}(n)$ e $P_{P O C}(n), n \geq 3$, segue da propriedade transitiva. Considere a matriz $M$ abaixo, cujas colunas são indexadas pelas arestas de $D_{3}$, isto é,

$$
\begin{array}{r}
(i, j) \quad(j, k) \\
M=\left[\begin{array}{llllllr}
1 & 1 & 1 & 0 & 0 & 0 \\
1 & 1 & 1 & 1 & 0 & 0 \\
1 & 1 & 1 & 0 & 1 & 0 \\
1 & 1 & 1 & 1 & 1 & 1 \\
0 & 1 & 0 & 1 & 0 & 1 \\
1 & 0 & 0 & 0 & 1 & 1
\end{array}\right]
\end{array}
$$

É fácil ver que cada linha de $M$ é o vetor característico de uma pré-ordem completa de $D_{3}$ que satisfaz a inequação-triângulo com igualdade. Como $M$ tem posto completo, segue que a inequação-triângulo é uma faceta de $P_{P O C}(3)$.

Como toda pré-ordem completa é uma pré-ordem, esta mesma prova é suficiente para mostrar que a inequação-triângulo é uma faceta de $P_{P O}(3)$, uma vez que dim $P_{P O}(3)=$ $\operatorname{dim} P_{P O C}(3)$.

Pode-se verificar que a inequação-triângulo é uma faceta de $P_{O P}(3)$, observando que a matriz $N$ abaixo tem posto completo e cada linha de $N$ é o vetor característico de uma ordem parcial que satisfaz a inequação-triângulo com igualdade.

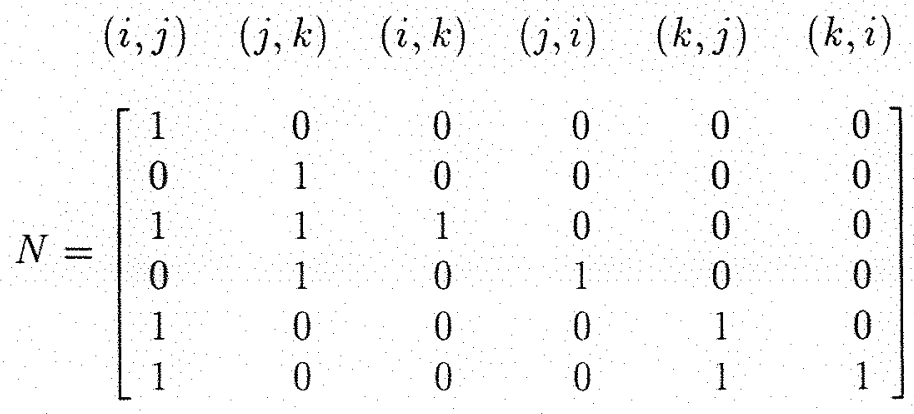


Para mostrar que a inequação-triângulo $x_{\alpha}+x_{\beta}-x_{\gamma} \leq 1$ é uma faceta de $P_{P O}(n)$, $P_{O P}(n)$ ou $P_{P O C}(n), n>3$, vamos denotá-la por $a^{T} x \leq 1$. É fácil construir uma ordem linear $A$ de $D_{n}$, que contém as arestas $\alpha, \beta$ e $\gamma$. Então, $a^{T} x^{A}=1$. Pelo lema da elevação, segue que a inequação-triângulo é uma faceta de $P_{P O}(n), P_{O P}(n)$ e $P_{P O C}(n)$, para todo $n, n \geq 3$.

\section{III.3.2 Facetas dos arcos paralelos}

Suponha que $\alpha$ seja uma aresta do digrafo $D_{n}$. Da propriedade total, segue a validade da inequação $x_{\alpha}+x_{\bar{\alpha}} \geq 1$ para $P_{P O C}(n)$; e da propriedade anti-simétrica, a validade de $x_{\alpha}+x_{\bar{\alpha}} \leq 1$, para $P_{O P}(n)$. Note que ambas as inequações não sầo válidas para $P_{P O}(n)$ e que a equação $x_{\alpha}+x_{\bar{\alpha}}=1$ é obviamente válida para $P_{O L}(n), n \geq 2$. Reinelt [1985] provou que $x_{i, j}+x_{j, i}=1$ para $1 \leq i<j \leq n$ constitui um sistema minimal de equaçôes de $P_{O L}(n)$.

Teorema III.7: As afirmaçôes abaixo sâo verdadeiras para toda aresta $\alpha \in A_{n}, n \geq 2$ :

(i) $x_{\alpha}+x_{\bar{\alpha}} \geq 1$ é uma faceta de $P_{P O C}(n)$;

(ii) $x_{\alpha}+x_{\bar{\alpha}} \leq 1$ é uma faceta de $P_{O P}(n)$.

Prova: Pelo lema da elevação, é suficiente mostrar o resultado para $P_{P O C}(2)$ e $P_{O P}(2)$, respectivamente. Isto é facilmente verificado, com os vetores característicos dos subdigrafos $G_{1}$ e $G_{2}$ de $D_{2}$ tais que $A G_{1}=\{\alpha\}$ e $A G_{2}=\{\bar{\alpha}\}$. 
Uma generalização da inequação $x_{\alpha}+x_{\bar{\alpha}} \leq 1$, que surge naturalmente na descrição de politopos associados a problemas envolvendo grafos dirigidos acíclicos é aquelas que exclui os vetores de incidência correspondentes a circuitos dirigidos. Considere $C$ o conjunto das arestas de um circuito dirigido de $D_{n}$. Então a inequação

$$
x(C) \leq|C|-1
$$

é denominada inequação do $k$-circuito, sendo $|C|=k$.

Esta inequação é obviamente válida para $P_{O P}(n)$ e $P_{O L}(n), n \geq k$. Reinelt [1985] mostrou que a inequação do $k$-circuito é uma faceta de $P_{O L}(n)$ se e somente se $k=3$. Jünger [1985] provou que a inequação do $k$-circuito é uma faceta de $P_{A C}(n)$, sendo este politopo definido como o fecho convexo dos vetores característicos de subdigrafos acíclicos de $D_{n}$. O teorema abaixo mostra que a inequação do $k$-circuito não define uma faceta de $P_{O P}(n), n \geq k$.

Teorema III.8: Seja $C$ um k-circuito dirigido $\mathrm{em} D_{n}, n \geq k>2$. Entâa a inequação

$$
x(C) \leq k-1
$$

é válida, mas não define uma faceta de $P_{O P}(n)$.

Prova: Se $k=3$, considere s.p.g., o circuito $C$ com arestas $(1,2),(2,3)$ e $(3,1)$. A inequação $x(C) \leq 2$ é a soma das seguintes inequações-facetas de $P_{O P}(n)$ :

$$
\begin{aligned}
x_{1,2}+x_{2,3}-x_{1,3} & \leq 1 \\
x_{1,3}+x_{3,1} & \leq 1 .
\end{aligned}
$$


Logo, $x(C) \leq 2$ pode ser obtida como combinação linear de inequações-facetas de $P_{O P}(n)$, e portanto não define uma faceta de $P_{O P}(n), n \geq 3$.

Suponha agora que $k>3$ e considere, s.p.g., $C=<(1,2),(2,3), \ldots(k, 1)>$. Seja $C^{\prime}$ o circuito dirigido $\langle(2,3), \ldots,(k-1, k),(k, 2)>$. Somando as inequações

$$
\begin{array}{ll}
x_{k, 1}+x_{1,2}-x_{k, 2} \leq 1 & \text { (inequação-triângulo) } \\
x_{2,3}+x_{3,4}+\cdots+x_{k-1, k}+x_{k, 2} \leq k-2, & \text { (inequação do }(k-1) \text {-circuito) }
\end{array}
$$

obtemos a inequação do $k$-circuito. Se $\left|C^{\prime}\right|>3$, podemos repetir este procedimento, concluindo que a inequação do $k$-circuito pode ser obtida como combinação linear de $k-3$ inequações-triângulo e uma inequação 3-circuito. Logo, ela não define uma faceta de $P_{O P}(n), n \geq k$.

\section{III.3.3 Facetas triviais}

É imediato que para todo $\alpha \in A_{n}$, as inequaçôes triviais $0 \leq x_{n} \leq 1$ são sempre válidas para politopos definidos como fecho convexo de vértices cujos componentes são zero e um. O teorema abaixo nos responde para quais dos três politopos em estudo, estas inequaçôes definem facetas.

Teorema III.9: Para toda aresta $\alpha \in A_{n}, n \geq 2$,

(i) $x_{\alpha} \leq 1$ é faceta de $P_{P O}(n)$ e de $P_{P O C}(n)$, mas nâo de $P_{O P}(n)$;

(ii) $x_{\alpha} \geq 0$ é faceta de $P_{P O}(n)$ e de $P_{O P}(n)$, mas não de $P_{P O C}(n)$.

Prova: Basta provar os resultados para $n=2$, uma vez que é trivial contruir ordens lineares em $D_{n}$ que contêm a aresta $\alpha$ (para o caso (i)) ou que contêm a aresta $\bar{\alpha}$ (para o caso (ii)) e estender o resultado para $n>2$, usando o lema da elevação. 
Considere subdigrafos geradores $G_{i}$ de $D_{2}$, para $1 \leq i \leq 4$, sendo $A G_{1}=\emptyset, A G_{2}=$ $\{\alpha\}, A G_{3}=\{\alpha, \bar{\alpha}\}$ e $A G_{4}=\{\bar{\alpha}\}$. Os vetores de incidencia dos grafos $G_{2}$ e $G_{3}$ mostram que $x_{\alpha} \leq 1$ é faceta de $P_{P O}(2)$ e de $P_{P O C}(2)$, enquanto que os vetores de incidência de $G_{1}$ e de $G_{4}$ provam que $x_{\alpha} \geq 0$ é faceta de $P_{P O}(2)$ e de $P_{O P}(2)$.

Por outro lado, a inequação $x_{\alpha} \leq 1$ pode ser obtida como combinação linear das inequações $-x_{\bar{\alpha}} \leq 0$ e $x_{\alpha}+x_{\bar{\alpha}} \leq 1$, facetas de $P_{O P}(n)$. Logo, $x_{\alpha} \leq 1$ não é faceta de $P_{O P}(n)$.

Da mesma forma, a inequação $x_{\alpha} \geq 0$ não é faceta de $P_{P O C}(n)$, pois pode ser obtida como combinação linear das inequações-facetas de $P_{P O C}(n),-x_{\bar{\alpha}} \geq-1$ e $x_{\alpha}+x_{\bar{\alpha}} \geq 1$.

\section{III.3.4 Facetas dos circuitos ímpares com 2-cordas}

Nesta e nas próximas seçôes, tanto indicamos um circuito pela seqüência ou pelo conjunto de suas arestas ou de seus vértices.

Definição III.10: Seja $G$ um digrafo com $A G=C \cup C O$, sendo $C$ o conjunto das arestas de um circuito hamiltoniano em $G$ e $C O$ o conjunto das 2-cordas de $C$. Suponha também que $|V G|=n \geq 4$. Então a inequaçào induzida por $G$

$$
x(C)-x(C O) \leq\left\lfloor\frac{n}{2}\right\rfloor,
$$

é denominada inequação do $n$-circuito com 2 -cordas.

Note que $D_{5}$ induz 24 inequações distintas do tipo acima, uma para cada circuito hamiltoniano de $D_{5}$. Neste caso, se $C=\langle(1,2),(2,3),(3,4),(4,5),(5,1)\rangle$, então $C O=\{(i,(i+2) \bmod 5): 1 \leq i \leq 5\}$ é o conjunto das 2 -cordas de $C$, que por sua vez é um circuito hamiltoniano de $D_{5}$. Assim, algumas das inequações dos 5 -circuitos com 
2-cordas induzidas por subdigrafos de $D_{5}$ são

$$
\begin{aligned}
& x(C)-x(C O) \leq 2 \\
& x(\bar{C})-x(\overline{C O}) \leq 2 \\
& x(C O)-x(\bar{C}) \leq 2 \\
& x(\overline{C O})-x(C) \leq 2 .
\end{aligned}
$$

Observe que qualquer uma das inequações acima é induzida por um digrafo isomorfo ao digrafo abaixo:

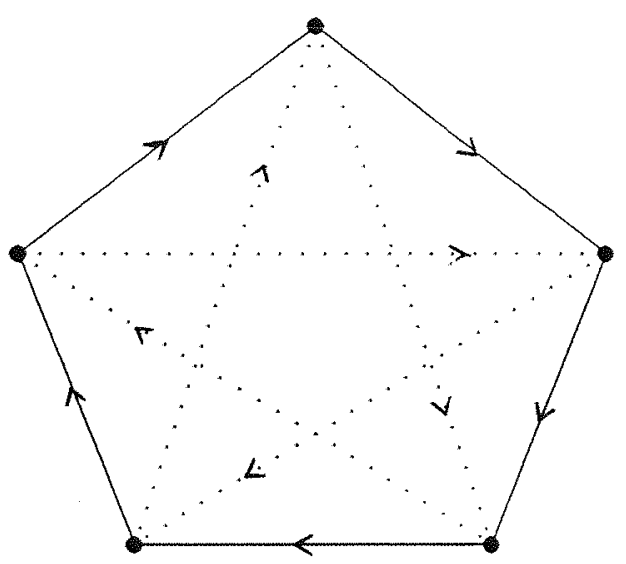

Teorema III.11: Seja $G$ um digrafo de: $D_{n}$ com $A G=C \cup C O$, sendo $C$ um circuito hamiltoniano, CO o conjunto das Q -cordas de $C \in \mid V(i \mid=n \geq 4$. Então a inequaçào do n-circuito com 2-cordas induzida por $G, F C C(n)$,

$$
x(C)-x(C O) \leq\left\lfloor\frac{n}{2}\right\rfloor
$$

é válida para $P_{P O}(n), P_{P O C}(n)$ e $P_{O P}(n)$. Além disso. cla r uma faceta de $P_{P O}(n)$ e de $P_{O P}(n)$ se e somente se $n$ for impar.

Prova: Suponha, s.p.g., que $V G=\{1,2, \ldots, n\}$ e $C=<(1,2),(2,3), \ldots,(n-1, n)$, $(n, 1)>$. Então $C O=\{(1,3),(2,4), \ldots,(n-1,1),(n, 2)\}$. Para estabelecer a validade de $F C C(n)$ para os três politopos em estudo, procedemos da seguinte forma: para cada 
aresta $\alpha \in C O$, consideramos as duas arestas $\beta$ e $\gamma$ em $C$, tais que $G[\{\beta, \gamma, \alpha\}]$ induz a seguinte inequação-triângulo:

$$
x_{\beta}+x_{\gamma}-x_{\alpha} \leq 1 .
$$

Somamos as $n$ inequações obtidas desta forma para cada $\alpha \in C O$, obtendo

$$
2 x(C)-x(C O) \leq|C O|=n
$$

Como a inequação

$$
-x(C O) \leq 0
$$

é válida para $P_{O P}(n), P_{P O C}(n)$ e $P_{P O}(n)$, adicionando-a à inequação (1) e dividindo o resultado por 2 , obtemos a inequaçào válida:

$$
x(C)-x(C O) \leq \frac{n}{2}
$$

Observe que para qualquer vértice de $P_{P O C}(n), P_{O P}(n)$ ou $P_{P O}(n)$, o lado esquerdo da inequação acima é um inteiro. Portanto, podemos arredondar o lado direito para o maior inteiro menor ou igual a $\frac{n}{2}$, sem perder a validade da inequação resultante. Conclúmos assim que $F C C(n)$ é válida para os três politopos em questão.

Note que, se $n$ for par, fica claro que $F C C(n)$ foi obtida por combinaşào linear de inequaçoos válidas e portanto não define uma faceta.

Suponha agora que $n$ é ímpar, $n=2 k+1, k \geq 2$. Vamos provar incialmente que FCC $(n)$ é faceta de $P_{O P}(n)$. Para isto, considere todas as adições módulo $2 k+1$.

Vamos denotar por $a^{T} x \leq a_{0}$ a inequaçầo $x(C)-x(C O) \leq k$.

Seja $F_{a}=\left\{x \in P_{O P}(n): a^{T} x=a_{0}\right\}$, e suponha que $b^{T} x \leq b_{0}$ é uma inequaçà-faceta de $P_{O P}(n)$ tal que:

$$
F_{a} \subseteq F_{b}=\left\{x \in P_{O P}(n): b^{T} x=b_{0}\right\} .
$$

O próximo passo será mostrar que existe $\lambda \in \mathbb{R}^{*}$ tal que $b=\lambda a$. 
Para cada $i \in V G$, seja $P_{i}$ um $k$-emparelhamento contido em $C \backslash\{(i, i+1),(i, i-1)\}$,

$$
P_{i}=\{(i+1, i+2),(i+3, i+4), \ldots,(i+2 k-1, i+2 k)\} .
$$

É fácil ver que

$$
x^{P_{i}} \in F_{a} \subseteq F_{b} \text { para todo } i \in V G,
$$

e portanto,

$$
b^{T} x^{P_{1}}=b^{T} x^{P_{2}}=\cdots=b^{T} x^{P_{2 k+1}}=b_{0} .
$$

Observe que $P_{i} \Delta P_{i+2}=\{(i, i+1),(i+1, i+2)\}$. Este fato, juntamente com a igualdade $b^{T} x^{P_{i}}=b^{T} x^{P_{i+2}}$ implica que $b_{i, i+1}=b_{i+1, i+2}$. Portanto, podemos concluir que existe $\lambda \in \mathbb{R}$ tal que

$$
b_{\alpha}=\lambda, \quad \text { para toda aresta } \alpha \in C
$$

Agora, para cada $i \in V G$, considere

$$
Q_{i}=P_{i} \cup\{(i, i+1),(i, i+2)\}
$$

Claramente,

$$
x^{Q_{1}} \in F_{a} \subseteq F_{b} \text { para todo } i \in V G
$$

Como $x^{P_{i}} \in F_{b}$, segue que

$$
b^{T} x^{P_{\mathbf{i}}}=b^{T} x^{Q}
$$

e portanto, $b_{i, i+1}+b_{i, i+2}=0$. De (2), podemos concluir que $b_{i, i+2}=-\lambda$, donde

$$
b_{\beta}=-\lambda \text { para todo } \beta \in C O
$$

É fácil concluir que

$$
b_{\bar{\alpha}}=0 \text { para todo } \bar{\alpha} \in \bar{C} .
$$


Para isto, basta tomar as ordens parciais $P_{i} \cup\{(i+1, i)\}$ e efetuar comparações, como anteriormente. Também temos que

$$
b_{\bar{\beta}}=0 \text { para todo } \bar{\beta} \in \overline{C O} \text {. }
$$

Para verificar este fato, tome, para $i \in V G$, a ordem parcial

$$
Q_{i}=P_{i} \cup\{(i+1, i),(i+2, i)\}
$$

De $x^{P_{i}}, x^{Q_{i}} \in F_{a} \subseteq F_{b}$, vem que

$$
b^{T} x^{Q_{i}}=b^{T} x^{P_{i}}
$$

donde $b_{i+1, i}+b_{i+2, i}=0$. Usando o fato (5), concluímos que

$$
b_{i+2, i}=0
$$

O próximo passo é mostrar que $b_{\gamma}=0$ para todo $\left.\gamma \in A_{n} \backslash(C \cup C O \cup \bar{C} \cup \overline{C O})\right)$. Para tanto, considere $k>2$ e tome o conjunto $J=\{3,5, \ldots, 2 k-3\}$.

Observe que as arestas de $\left.A_{n} \backslash(C \cup C O \cup \bar{C} \cup C \bar{O})\right)$ sào precisamen te as do tipo $(i, i+j)$, $(i+j, i),(i, i+j+1),(i+j+1, i)$ para $i \in V G, j \in J$.

Para $i \in V G$, considere os seguintes conjuntos de arestas:

$$
\begin{aligned}
& S_{1}^{i}=P_{i} \cup\{(i+j, i)\}, \\
& S_{2}^{i}=P_{i} \cup\{(i+j, i),(i+j+1, i)\}, \\
& S_{3}^{i}=P_{i} \cup\{(i+j, i),(i, i+j+1)\} \quad \mathrm{e} \\
& S_{4}^{i}=P_{i} \cup\{(i, i+j),(i, i+j+1)\} .
\end{aligned}
$$

Note que $S_{\ell}^{i}, 1 \leq \ell \leq 4$, são ordens parciais tais que $x^{S_{\ell}^{i}} \in F_{a} \subseteq F_{b}$. Como $x^{P_{i}} \in F_{b}$, temos que:

$$
\begin{aligned}
& b^{T} x^{P_{i}}=b^{T} x^{S_{1}^{2}}, \text { donde } b_{i+j, i}=0, \\
& b^{T} x^{S_{1}^{i}}=b^{T} x^{S_{2}^{2}}, \text { donde } b_{i+j+1, i}=0,
\end{aligned}
$$




$$
\begin{aligned}
& b^{T} x^{S_{1}^{i}}=b^{T} x^{S_{3}^{i}}, \quad \text { donde } b_{i, i+j+1}=0, \quad \mathrm{e} \\
& b^{T} x^{S_{3}^{i}}=b^{T} x^{S_{4}^{i}}, \quad \text { donde } b_{i+j, i}=-b_{i, i+j} .
\end{aligned}
$$

Como $b_{i+j, i}=0$ segue que $b_{i, i+j}=0$.

Reunindo todos os casos, concluímos que existe $\lambda \in \mathbb{R}$ tal que

$$
b_{\alpha}=\left\{\begin{aligned}
\lambda & , \text { se } \alpha \in C \\
-\lambda & , \text { se } \alpha \in C O \\
0 & , \text { se } \alpha \in A_{n} \backslash C \cup C O
\end{aligned}\right.
$$

isto é, $b_{\alpha}=\lambda a_{\alpha}$ para toda aresta $\alpha \in A_{n}$ e $b^{T} x \leq b_{0}$ pode ser escrita como

$$
\lambda(x(C)-x(C O)) \leq \lambda k
$$

Logo $b=\lambda a$, para $\lambda \in \mathbb{R}^{*}$, e portanto pelo Teorema I.6, concluímos que $F C C(n)$ é uma faceta de $P_{O P}(n)$.

Como toda ordem parcial é uma pré-ordem e $\operatorname{dim}\left(P_{O P}(n)\right)=\operatorname{dim}\left(P_{P O}(n)\right)$, concluímos que $\mathrm{FCC}(n)$ é também uma faceta de $P_{F^{\prime}}(n)$.

Para mostrar que a inequação $x(C)-x(C O) \leq\left\lfloor\frac{n}{2}\right\rfloor$ define uma faceta de $P_{P O}(m)$ ou $P_{O P}(m)$, para todo $m, m>n$, vamos provar que a condição do lema da elevação está satisfeita, através do seguinte resultado:

Teorema III.12: Seja $G$ como no teorema anterior, sendo $|V G|=n=2 k+1, k \geq 2$. Existe uma ordem linear $A$ de $D_{n}$ tal que

$$
x^{A}(C)-x^{A}(C O)=k
$$

sendo $x(C)-x(C O) \leq k$ a inequação do n-circuito com d-cordas. 
Prova: Suponha que $V G=\{1,2, \ldots, 2 k+1\}$ e que $C=<(1,2),(2,3), \ldots,(2 k, 2 k+1)$, $(2 k+1,1)>$. Então

$$
C O=<(1,3),(2,4), \ldots,(2 k-1,2 k+1),(2 k, 1),(2 k+1,2)>.
$$

Considere a ordem linear $O=<1,2 k, 2 k-2, \ldots, 2,2 k+1,2 k-1, \ldots, 3>$ de $D_{n}$, e seja $A=A(O)$. Como no teorema anterior, todas as operações serão realizadas módulo $2 k+1$.

Inicialmente, vamos mostrar que $|C \cap A|=k+1$. Para isto, considere $C \cap A=A_{1} \cup A_{2}$, sendo

$$
\begin{aligned}
& A_{1}=\{(i, i+1) \in A, \quad i \text { é mpar }\} \text { e } \\
& A_{2}=\{(i, i+1) \in A: \quad i \text { épar }\}
\end{aligned}
$$

É fácil ver que $\left|A_{1}\right|=1$, pois na sequêencia $O$ todos os vértices impares ocorrem após todos os vértices pares, com exceção do vértice 1. Este mesmo fato é suficiente para concluirmos que $\left|A_{2}\right|=k$.

O próximo passo será provarmos que $|C O \cap A|=1$, concluindo assim que $x^{A}(C)-$ $x^{A}(C O)=k$. Suponha então que exista $i \in V G$ tal que $(i, i+2) \in C O \cap A$. Se $1 \leq i \leq 2 k-1$, como $i$ e $i+2$ têm a mesma paridade, observando que na seqüência $O$ os vértices pares ocorrem em ordem decrescente, segue que $i$ é ímpar. Neste caso, a única possibilidade é $(i, i+2)=(1,3)$, já que os vértices ímpares maiores que 1 ocorrem em ordem decrescente. Obviamente $(2 k, 1) \notin A$ e $(2 k+1,2) \notin A$. Portanto, $|C O \cap A|=1$, como queríamos provar.

Teorema III.13: Seja $G$ definido como no teorema III.11. Então

$$
x(C)-x(C O) \leq\left\lfloor\frac{|C|}{2}\right\rfloor
$$

é uma inequaçâo válida para $P_{O P}(m), P_{P O}(m), m \geq|C| \geq 4$ e define uma faceta desses politopos se e somente se $|C|$ é impar. 
Observação: Veremos mais adiante que a inequação do 5-circuito com 2-cordas não é uma faceta de $P_{P O C}(n)$, para todo $n \geq 5$.

\section{III.3.5 Facetas dos pentágonos}

Nesta secção, todas as adições e subtrações devem ser consideradas módulo 5 .

Definição III.14: Seja $G$ um digrafo com 5 vértices, tal que $A G=C \cup C O \cup \bar{C} \cup \overline{C O}$, onde $C$ é o conjunto das arestas de um circuito hamiltoniano em $G$ e $C O$ é o conjunto das 2-cordas de $C$. Então a inequação induzida por $G$,

$$
3 x(C)-3 x(C O)+x(\bar{C})-x(\overline{C O}) \leq 4
$$

é denominada inequação-pentágono.

Observe que $D_{5}$ induz 24 inequações-pentágonos diferentes, uma para cada circuito hamiltoniano. Para facilitar a notação, se $V G=\{1,2,3,4,5\}$, vamos convencionar que $C=\{(i, \imath+1): 1 \leq i \leq 5\}$. Na figura abaixo, para visualizarmos melhor a inequação-pentágono, indicamos nas arestas os coeficientes correspondentes a essas arestas na inequação induzida por $G$.

O objetivo desta seção é provarmos que a inequação-pentágono é faceta de $P_{P O C}(n)$, $n \geq 5$. Para provar a validade da inequação-pentágono, usaremos o fato de que a inequação

$$
x(C)-x(C O)+x(\bar{C})-x(\overline{C O}) \leq k
$$

é válida para $P_{P O C}(2 k+1)$. Esta inequação, denominada inequação do n-circuito duplo com 2-cordas, fez-nos pensar inicialmente, que havíamos encontrado uma classe bastante interessante de facetas para $P_{P O C}(n)$. Entretanto, obtivemos a comprovação de que a inequação do $n$-circuito duplo com 2-cordas não é faceta de $P_{P O C}(n)$, quando $n=5 \mathrm{e}$ 


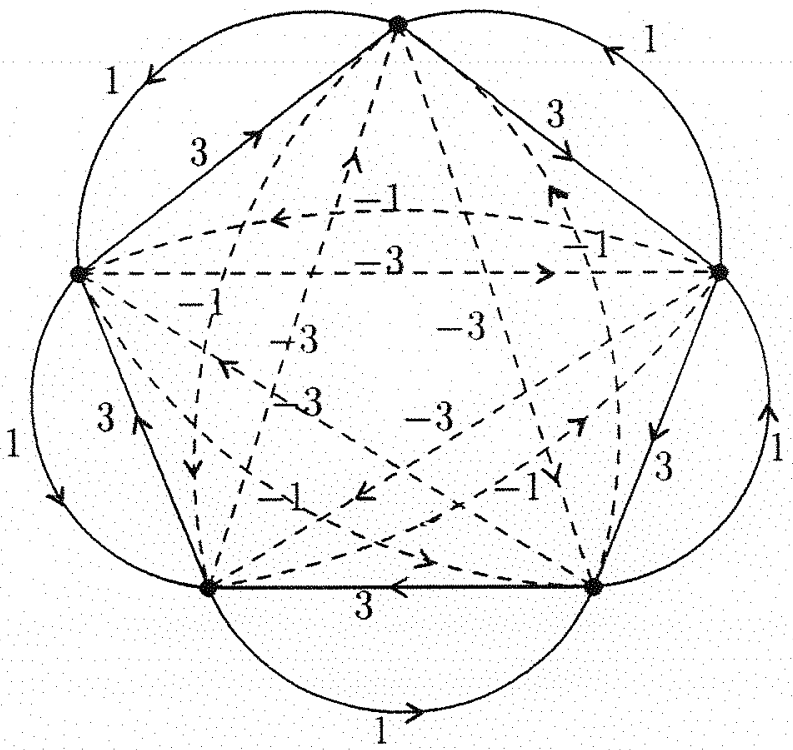

$n=7$. Foi porém, introduzindo pequenas variaçôes na inequação do 5 -circuito duplo com 2-cordas, que obtivemos a inequação-pentágono.

Proposição III.15: A inequação do n-circuito duplo com 2-cordas, definida como,

$$
x(C)-x(C O)+x(\bar{C})-x(\overline{C O}) \leq k
$$

é válida para $P_{P O C}(n)$, sendo $n=2 k+1$.

Prova: Nesta prova, fica subentendido que o politopo em consideração é $P_{P O C}(n)$. Somando inequações-triângulo, obtemos a validade das inequaçôes abaixo, conforme foi visto na prova do Teorema III.11.

$$
\begin{aligned}
& 2 x(C)-x(C O) \leq 2 k+1 \\
& 2 x(\bar{C})-x(\overline{C O} \leq 2 k+1
\end{aligned}
$$

Da propriedade total, obtém-se a validade da inequação

$$
-x(C O)-x(\overline{C O}) \leq-(2 k+1)
$$


Somando as inequações (1), (2) e (3), obtemos:

$$
2 x(C)+2 x(\bar{C})-2 x(C O)-2 x(\overline{C O}) \leq 2 k+1 .
$$

Dividindo ambos os lados da inequação (4) por 2 e arredondando o lado direito para o maior inteiro menor ou igual a $(2 k+1) / 2$, obtemos a inequação do $n$-circuito duplo com 2-cordas.

Teorema III.16: Seja $a^{T} x \leq a_{0}$ a inequaçấo-pentágono

$$
3 x(C)-3 x(C O)+x(\bar{C})-x(\overline{C O}) \leq 4
$$

induzida por um digrafo $G$, como na definição III.14. As seguintes afirmaşôes sâo verdadeiras:

(i) $a^{T} x \leq a_{0}$ é válida para $P_{P O C}(5)$;

(ii) $a^{T} x \leq a_{0}$ é uma faceta de $P_{P O C}(5)$;

(iii) $a^{T} x \leq a_{0}$ é uma faceta de $P_{P O C}(n)$, para todo $n>5$.

\section{Prova:}

(i) Esta demonstração será feita através de combinações e arredondamento de inequações válidas para $P_{P O C}(5)$.

Da propriedade total, segue a validade de

$$
-x(C O)-x(\overline{C O}) \leq-5
$$

Das inequações-triângulo, obtém-se a validade de

$$
\begin{gathered}
2 x(C)-x(C O) \leq 5 \quad \\
x(\bar{C})+x(\overline{C O})-x(C O) \leq 5 .
\end{gathered}
$$


Somando as inequações abaixo,

$$
\begin{aligned}
2 x(C)-x(C O) & \leq 5 \quad \mathrm{e} \\
-x(C O) & \leq 0
\end{aligned}
$$

dividindo o resultado por 2 e arredondando o lado direito para o maior inteiro menor ou igual a $5 / 2$, obtemos a inequação (4), também válida para $P_{P O C}(5)$ :

$$
x(C)-x(C O) \leq 2
$$

Somando 2 vezes a inequação do 5 -circuito duplo com 2-cordas,

$$
2 x(C)-2 x(C O)+2 x(\bar{C})-2 x(\overline{C O}) \leq 4,
$$

com as inequações abaixo,

$$
\begin{aligned}
6 x(C)-3 x(C O) & \leq 15 \\
-x(C O)+x(\bar{C})+x(\overline{C O}) & \leq 5 \\
-2 x(C O) & -2 x(\overline{C O}) \\
x(C)-x(C O) & \leq-10 \\
& \leq 2
\end{aligned}
$$

obtemos a inequação

$$
9 x(C)-9 x(C O)+3 x(\bar{C})-3 x(\overline{C O}) \leq 16 .
$$

Dividindo esta inequação por 3 e arredondando, obtemos:

$$
3 x(C)-3 x(C O)+x(\bar{C})-x(\overline{C O}) \leq 5 .
$$

Adicionando a inequação (5) à inequação do 5 -circuito duplo com 2-cordas, dividindo o resultado por 2 e arredondando, obtemos:

$$
2 x(C)-2 x(C O)+x(\bar{C})-x(\overline{C O}) \leq 3
$$

Considere agora o seguinte conjunto de inequações, obviamente válidas para $P_{P O C}(5)$, pois são combinações lineares de inequações anteriormente discutidas: 


$$
\begin{array}{rll}
8 x(C)-8 x(C O)+4 x(\bar{C})-4 x(\overline{C O}) & \leq 12 \\
3 x(C)-3 x(C O)+x(\bar{C})-x(\overline{C O}) & \leq 5 \\
6 x(C)-3 x(C O)+ & \leq 15 \\
-x(C O)+x(\bar{C})+x(\overline{C O}) & \leq 5 \\
-2 x(C O)+-2 x(\overline{C O}) & \leq-10 \\
x(C)-x(C O) & \leq 2
\end{array}
$$

Somando-as, obtemos a inequação:

$$
18 x(C)-18 x(C O)+6 x(\bar{C})-6 x(\overline{C O}) \leq 29
$$

Dividindo ambos os termos por 6 e arredondando, obtemos a inequação-pentágono. Logo, ela é válida para $P_{P O C}(5)$.

(ii) Para mostrar que a inequação-pentágono define uma faceta de $P_{P O C}(5)$, suponha s.p.g., que $V G=\{1,2,3,4,5\}$ e que $C=\{(i, i+1): 1 \leq i \leq 5\}$. Denote por $F_{a}$ a face definida por $a^{T} x \leq a_{0}$ e seja $F_{b}$ una faceta de $P_{P O C}(5)$ tal que:

$$
F_{a}=\left\{x \in P_{P O C}(5): a^{T} x=a_{0}\right\} \subseteq F_{b}=\left\{x \in P_{P O C}(5): b^{T} x=b_{0}\right\}
$$

Vamos provar que existe $\lambda \in \mathbb{R}^{*}$ tal que $b=\lambda a$.

Inicialmente, para cada $i \in V G$, seja $O_{i}$ a ordem linear de $D_{5}$ representada por $<i, i+1, i+2, i+3, i+4>$. Considere os seguintes conjuntos de arestas:

$$
\begin{aligned}
& M_{i}=A\left(O_{i}\right) \cup\{(i+1, i),(i+3, i+2)\} \mathrm{e} \\
& M_{i}^{\prime}=A\left(O_{i}\right) \cup\{(i+1, i),(i+4, i+3)\} .
\end{aligned}
$$

É fácil verificar que

$$
x^{M_{i}}, x^{M_{i}^{\prime}} \in F_{a} \subseteq F_{b} \text { para todo } i \in V G \text {. }
$$

Portanto,

$$
b^{T} x^{M_{i}}=b^{T} x^{M_{i}^{\prime}}, \quad \text { para todo } i \in V G
$$


Como $M_{i} \Delta M_{i}^{\prime}=\{(i+3, i+2),(i+4, i+3)\}$, concluímos das igualdades acima que

$$
b_{i+3, i+2}=b_{i+4, i+3} \quad \text { para } i=1,2,3,4,5 \text {. }
$$

Logo, $b_{4,3}=b_{5,4}=b_{1,5}=b_{2,1}=b_{3,2}=\lambda$, para algum $\lambda \in \mathbb{R}$. Portanto,

$$
b_{\alpha}=\lambda \quad \text { para todo } \alpha \in \bar{C} .
$$

Considere agora, para cada $i \in V G$ o seguinte conjunto de arestas:

$$
M_{i}^{\prime \prime}=M_{i} \cup\{(i+4, i+3),(i+4, i+2)\} .
$$

Como $x^{M_{i}^{\prime \prime}} \in F_{a} \subseteq F_{b}$, concluímos que:

$$
b^{T} x^{M_{i}}=b^{T} x^{M_{i}^{\prime \prime}}
$$

donde $b_{i+4, i+2}=-b_{i+4, i+3}=-\lambda$.

Deste modo, obtemos

$$
b_{5,3}=b_{4,2}=b_{3,1}=b_{2,5}=b_{1,4}=-\lambda \text {, }
$$

donde

$$
b_{\alpha}=-\lambda \quad \text { para todo } \alpha \in \overline{C O}
$$

Com raciocínio análogo, obtém-se, para algum $\mu \in \mathbb{R}$ :

$$
\begin{aligned}
& b_{\beta}=\mu \text { para toda aresta } \beta \in C \\
& \text { e } b_{\beta}=-\mu \text { para todo } \beta \in C O
\end{aligned}
$$

A inequação $b^{T} x \leq b_{0}$ pode ser então reescrita como:

$$
\mu x(C)-\mu x(C O)+\lambda x(\bar{C})-\lambda x(\overline{C O}) \leq b_{0}=\lambda+\mu .
$$

Considere agora a ordem linear $0=<2,5,3,1,4>$ e o conjunto $A=A(O)$, indicado na figura abaixo. 


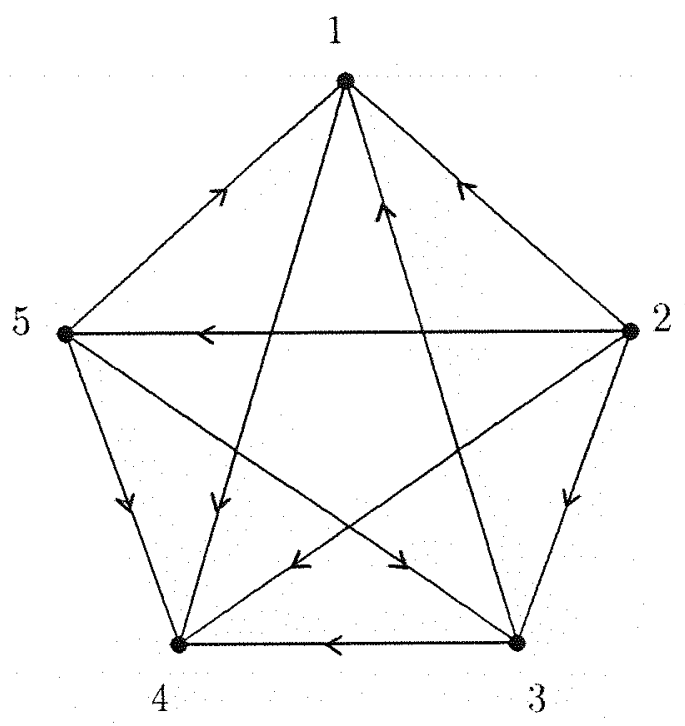

Por inspeção pode-se verificar que $x^{A} \in F_{a}$. Como $F_{a} \subseteq F_{b}$, de (7) podemos concluir que

$$
2 \mu-2 \lambda=\lambda+\mu
$$

donde $\mu=3 \lambda$.

Portanto, $b^{T} x \leq b_{0}$ pode ser escrita como

$$
3 \lambda x(C)-3 \lambda x(C O)+\lambda x(\bar{C})-\lambda x(\overline{C O}) \leq 4 \lambda .
$$

Logo, $b=\lambda a$ para $\lambda \in \mathbb{R}^{*}$ e, portanto, pelo teorema $1.6, a^{T} x \leq a_{0}$ é uma faceta de $P_{P O C}(5)$, como queríamos provar.

(iii) A prova de que a inequaçào-pentágono é uma faceta de $P_{P O C}(n), n>5$, segue usando o lema da elevação.

Corolário III.17: A inequação do 5-circuito com 2-cordas,

$$
x(C)-x(C O) \leq 2,
$$


não define uma faceta de $P_{P O C}(n), n \geq 5$.

Prova: Basta observar que $x(C)-x(C O) \leq 2$ pode ser obtida dividindo-se a soma das inequações abaixo por 7. Como a validade dessas inequações para $P_{P O C}(n), n \geq 5$, foi verificada anteriormente, segue que a inequação do 5-circuito com 2-cordas nâo define uma faceta de $P_{P O C}(n)$.

$$
\begin{array}{rr}
3 x(C)-3 x(C O)+x(\bar{C})-x(\overline{C O}) & \leq 4 \\
-x(C)+2 x(\overline{C O}) & \leq 5 \\
6 x(C)-3 x(C O)-x(\bar{C})- & \leq 15 \\
-x(C)-x(C O)-5(\overline{C O}) \leq-5
\end{array}
$$

Corolário III.18: A inequaçâo do 5-circuito duplo com 2-cordas,

$$
x(C)-x(C O)+x(\bar{C})-x(\overline{C O}) \leq 2,
$$

não define uma faceta de $P_{P O C}(n), n \geq 5$.

Prova: Basta observar que a inequação do 5 -circuito duplo com 2-cordas pode ser obtida dividindo-se por 4 a soma das duas inequaçóes-pentágono abaixo:

$$
\begin{aligned}
& 3 x(C)-3 x(C O)+x(\bar{C})-x(\overline{C O}) \leq 4 \\
& x(C)-x(C O)+3 x(\bar{C})-3 x(\overline{C O}) \leq 4
\end{aligned}
$$

\section{III.3.6 Facetas dos heptágonos}

Nesta seção, todas as adições e subtrações devem ser consideradas módulo 7 .

Definição III.19: Seja $G$ um digrafo com $V G=\{1,2, \ldots, 7\}$ e $A G=C \cup C O \cup \bar{C} \cup$ $\overline{C O} \cup D \cup \bar{D}$, sendo $C=\{(i, i+1): i=1, \ldots, 7\}, C O=\{(i, i+2): i=1, \ldots, 7\} \mathrm{e}$ 
$D=\{(i, i+3): i=1, \ldots, 7\}$. Então a inequação induzida por $G$,

$$
4 x(C)-4 x(C O)+2 x(\bar{C})-2 x(\overline{C O})+x(D)-x(\bar{D}) \leq 9
$$

é denominada inequação-heptágono. Os conjuntos $C, C O$ e $D$ são denominados circuito externo, circuito das cordas e circuito das diagonais, respectivamente.

Observe que $D_{7}$ dá origem a 720 diferentes inequações-heptágono. Algumas delas, considerando-se os mesmos conjuntos acima podem ser escritas como:

$$
\begin{aligned}
& 4 x(C)-4 x(C O)+2 x(\bar{C})-2 x(\overline{C O})+x(D)-x(\bar{D}) \leq 9 \\
& 4 x(C O)-4 x(\bar{D})+2 x(\overline{C O})-2 x(D)+x(C)-x(\bar{C}) \leq 9 \\
& 4 x(D)-4 x(\bar{C})+2 x(\bar{D})-2 x(C)+x(C O)-x(\overline{C O}) \leq 9 \\
& 4 x(\bar{C})-4 x(\overline{C O})+2 x(C)-2 x(C O)+x(\bar{D})-x(D) \leq 9 \\
& 4 x(\overline{C O})-4 x(D)+2 x(C O)-2 x(\bar{D})+x(\bar{C})-x(C) \leq 9 \\
& 4 x(\bar{D})-4 x(C)+2 x(D)-2 x(\bar{C})+x(\overline{C O})-x(C O) \leq 9
\end{aligned}
$$

A inequação-heptágono surgiu como uma extensào da inequaçào-pentágono. Não conseguimos porém, generalizar este resultado para $n>7$. Como veremos adiante, as demonstrações do caso $n=7$ são exaustivas, desencorajando qualquer tentativa de generalização.

Teorema III.20: Seja $a^{T} x \leq 9$ a seguinte inequação-héptágono,

$$
4 x(C)-4 x(C O)+2 x(\bar{C})-2 x(\overline{C O})+x(D)-x(\bar{D}) \leq 9
$$

induzida por um digrafo $G, G \subseteq D_{7}$, como na definiçâo III.19. As seguintes afirmaçôes são verdadeiras:

(i) $a^{T} x \leq 9$ é válida para $P_{P O C}(7)$; 
(ii) $a^{T} x \leq 9$ é uma faceta de $P_{P O C}(7)$;

(iii) $a^{T} x \leq 9$ é uma faceta de $P_{P O C}(n)$ para todo $n>7$.

\section{Prova:}

(i) Infelizmente não conseguimos provar a validade da inequação heptágono para $P_{P O C}(7)$ através de combinações e arredondamentos de inequações válidas, como fizemos na maioria das vezes. Neste caso, mostramos que para todo digrafo $H \subseteq D_{7}$ tal que $x^{A H}$ é um vértice de $P_{P O C}(7)$, tem-se que $a^{T} x^{A H} \leq 9$. Dado que esta prova consiste na análise de vários casos e subcasos, decidimos incluíla no anexo.

(ii) De acordo com a definição III.19, VG=\{1,., 7$\}, C=\{(i, i+1): i=1, \ldots, 7\}$, $C O=\{(i, i+2): i=1, \ldots, 7\}$ e $D=\{(i, i+3): i=1, \ldots, 7\}$. Denote por $F_{a}$ a face definida por $a^{T} x \leq 9$ e seja $F_{b}$ uma faceta de $P_{P O C}(7)$, tal que

$$
F_{a}=\left\{x \in P_{P O C}(7): a^{T} x=9\right\} \subseteq F_{b}=\left\{x \in P_{P O C}(7): b^{T} x=b_{0}\right\}
$$

Vamos provar que existe $\lambda \in \mathbb{R}^{*}$ tal que $b=\lambda a$.

Para cada $i \in V G$, considere em $D_{7}$ a ordem linear $O_{i}=\langle i, i+1, \ldots, i+5, i+6\rangle$. Considere os seguintes conjuntos de arestas:

$$
\begin{aligned}
& M_{i}=A\left(O_{i}\right) \cup\{(i+1, i),(i+3, i+2),(i+5, i+4)\} \mathrm{e} \\
& M_{i}^{\prime}=A\left(O_{i}\right) \cup\{(i+1, i),(i+3, i+2),(i+6, i+5)\}
\end{aligned}
$$

Não é difícil ver que

$$
x^{M_{i}}, x^{M_{i}^{\prime}} \in F_{a} \subseteq F_{b} \quad \text { para todo } i \in V G \text {. }
$$

Portanto,

$$
b^{T} x^{M_{\mathrm{i}}}=b^{T} x^{M_{i}^{\prime}}, \text { para todo } i \in V G .
$$


Como $M_{i} \Delta M_{i}^{\prime}=\{(i+5, i+4),(i+6, i+5)\}$, concluímos das igualdades acima que

$$
b_{i+5, i+4}=b_{i+6, i+5} \quad \text { para } i=1, \ldots, 7 .
$$

Logo,

$$
b_{6,5}=b_{7,6}=b_{1,7}=b_{2,1}=b_{3,2}=b_{4,3}=b_{5,4} .
$$

Ou seja, para algum $\lambda \in \mathbb{R}$ temos que

$$
b_{\alpha}=\lambda \quad \text { para todo } \alpha \in \bar{C}
$$

Considere agora, para cada $i \in V G$, o conjunto de arestas

$$
M_{i}^{\prime \prime}=M_{i} \cup\{(i+6, i+5),(i+6, i+4)\}
$$

Como $x^{M_{i}^{\prime \prime}} \in F_{a} \subseteq F_{b}$, concluímos que

$$
b^{T} x^{M_{i}}=b^{T} x^{M_{i}^{\prime \prime}}
$$

donde $b_{i+6, i+5}=-b_{i+6, i+4}=\lambda$.

Logo,

$$
b_{\alpha}=-\lambda \text { para todo } \alpha \in \overline{C O} .
$$

Com raciocínio análogo, obtém-se, para algum $\mu \in \mathbb{R}$ :

$$
\begin{aligned}
& b_{\beta}=\mu \text { para todo } \beta \in C \quad \text { e } \\
& b_{\beta}=-\mu \text { para todo } \beta \in C O .
\end{aligned}
$$

Considere agora, para cada $i \in V G$, o seguinte conjunto de arestas:

$$
\begin{aligned}
M_{i}^{\prime \prime \prime}= & M_{i} \backslash\{(i+1, i+2),(i+1, i+3),(i, i+2),(i, i+3)\} \\
& \cup\{(i+2, i+1),(i+3, i+1),(i+2, i),(i+3, i)\} .
\end{aligned}
$$


As arestas do fecho transitivo dos grafos $H_{i}$ e $K_{i}$ abaixo correspondem às arestas $M_{i}$ e $M_{i}^{\prime \prime \prime}$, respectivamente:

$H_{i}$

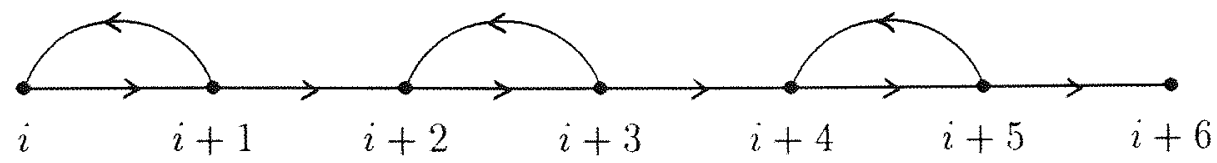

$K_{i}$

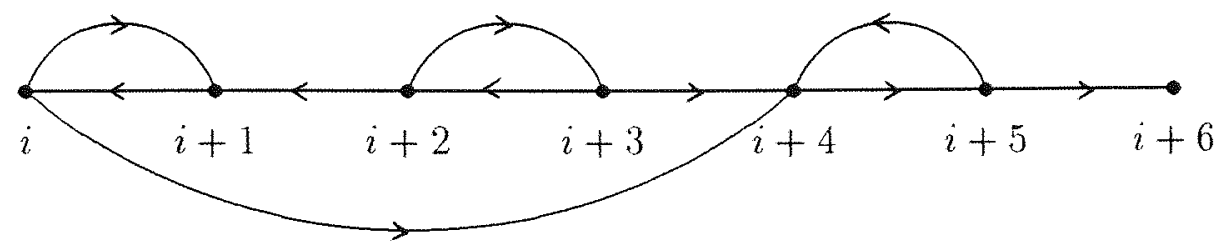

Como $x^{M_{i}^{\prime \prime}} \in F_{a}$ e $F_{a} \subseteq F_{b}$, segue que

$$
b^{T} x^{M_{i}}=b^{T} x^{M_{i}^{\prime \prime \prime}} .
$$

Logo,

$$
b_{i+1, i+2}+b_{i, i+2}+b_{i, i+3}+b_{i+1, i+3}=b_{i+2, i+1}+b_{i+2, i}+b_{i+3, i}+b_{i+3, i+1} .
$$

De (1), (2), (3) e (4), concluímos que

$$
b_{i, i+3}-b_{i+3, i}=\mu-\lambda, \quad \text { para } i=1, \ldots, 7 \text {. }
$$

Para cada $i \in V G$, considere agora o grafo $G_{i}$, indicado na figura abaixo:

$G_{i}$

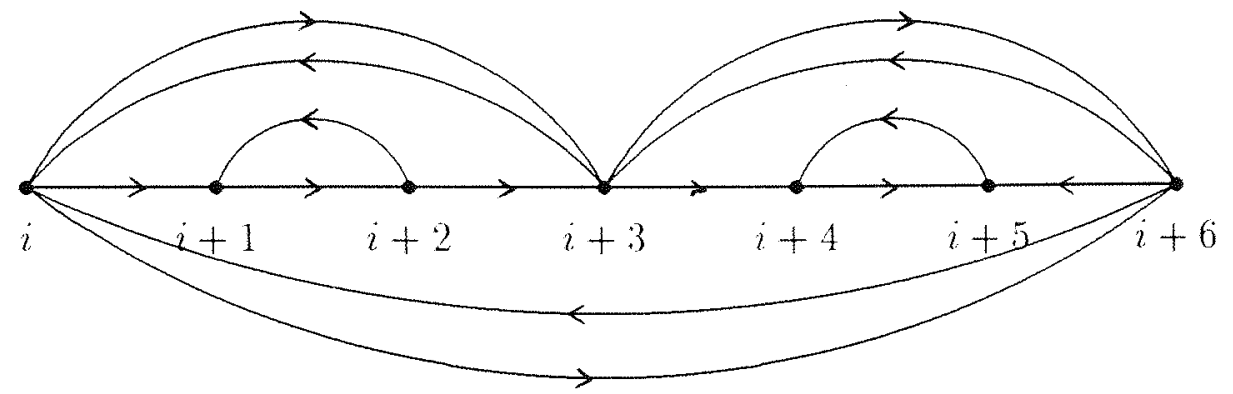


Sejam $N_{i}^{\prime}$ e $N_{i}^{\prime \prime}$ as arestas dos fechos transitivos dos grafos $G_{i}^{\prime}$ e $G_{i}^{\prime \prime}$, obtidos de $G_{i}$ como:

$$
\begin{aligned}
A G_{i}^{\prime} & =A G_{i}-\{(i+3, i),(i+3, i+6)\} \\
A G_{i}^{\prime \prime} & =A G_{i}-\{(i, i+3),(i+6, i+3)\} .
\end{aligned}
$$

De $\quad x^{A G_{i}}, x^{A G_{i}^{\prime}} \in F_{a}$ e $F_{a} \subseteq F_{b}$, vem que

$$
b_{i+3, i}=-b_{i+3, i+6} .
$$

De $\quad x^{A G_{i}}, x^{A G_{i}^{\prime \prime}} \in F_{a}$ e $F_{a} \subseteq F_{b}$, vem que

$$
b_{i, i+3}=-b_{i+6, i+3} .
$$

De (6), concluímos que

$$
b_{i, i+4}=-b_{i, i+3}
$$

Como $-b_{i, i+3}=b_{i+6, i+3}$ de $(7)$, segue que

$$
b_{i, i+4}=b_{i+6, i+3} .
$$

Então

$$
b_{1,5}=b_{7,4}=b_{6,3}=b_{5,2}=b_{4,1}=b_{3,7}=b_{2,6}=-\delta, \quad \text { para } \delta \in \mathbb{R} .
$$

Portanto,

$$
b_{\alpha}=-\delta \quad \text { para todo } \alpha \in \bar{D}
$$

De (8) e (10), concluímos que

$$
b_{c x}=\delta \quad \text { para todo } \alpha \in D
$$

De (5), $b_{\alpha}-b_{\bar{\alpha}}=\mu-\lambda$ para todo $\alpha \in D$. Portanto, $2 \delta=\mu-\lambda$, implicando $\delta=(\mu-\lambda) / 2$. 
A inequação $b^{T} x \leq b_{0}$ pode então ser reescrita como:

$$
\mu x(C)-\mu x(C O)+\lambda x(\bar{C})-\lambda x(\overline{C O})+\frac{\mu-\lambda}{2} x(D)-\frac{(\mu-\lambda)}{2} x(\bar{D}) \leq b_{0}
$$

sendo $b_{0}=(3 \mu+3 \lambda) / 2$.

Considere agora a ordem linear $O=<4,2,7,5,3,1,6>$ e o conjunto $T=O(A)$.

Veja na figura abaixo as arestas de $T$.

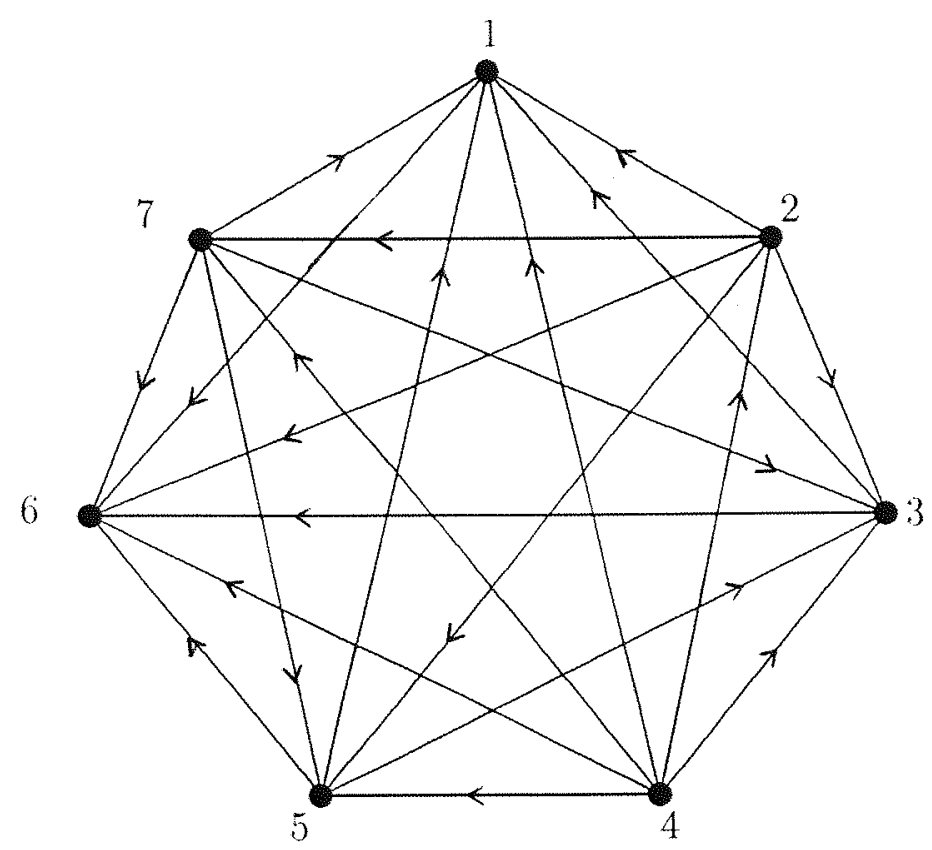

É fácil verificar que $x^{T} \in F_{a}$. Como $F_{a} \subseteq F_{b}$, segue que $x^{T} \in F_{b}$. Logo,

$$
3 \mu-3 \lambda+3 \delta=(3 \mu+3 \lambda) / 2
$$

donde $\mu=2 \lambda$. Então $b^{T} x \leq b_{0}$ pode ser reescrita como:

$$
2 \lambda x(C)-2 \lambda x(C O)+\lambda x(\bar{C})-\lambda x(\overline{C O})+\frac{\lambda}{2} x(D)-\frac{\lambda}{2} x(\bar{D}) \leq \frac{9 \lambda}{2} .
$$

Logo, $b=\lambda a$ para $\lambda=2$ e, pelo teorema $1.6, a^{T} x \leq 9$ é uma faceta de $P_{P O C}(T)$, como queríamos provar. 
(iii) A prova de que a inequação-heptágono é uma faceta de $P_{P O C}(n), n>7$, segue usando o lema da elevação. Note que no final da prova anterior exibimos uma ordem linear de $D_{7}$ que satisfaz as condições do lema da elevação.

Corolário III.21: A inequação do 7-circuito duplo com 2-cordas,

$$
x(C)-x(C O)+x(\bar{C})-x(\overline{C O}) \leq 3
$$

não define uma faceta de $P_{P O C}(7)$.

Prova: Basta observar que a inequação do 7-circuito duplo com 2-cordas pode ser obtida dividindo-se por 6 a soma das duas inequações-heptágono abaixo:

$$
\begin{aligned}
& 4 x(C)-4 x(C O)+2 x(\bar{C})-2 x(\overline{C O})+x(D)-x(\bar{D}) \leq 9 \\
& 2 x(C)-2 x(C O)+4 x(\bar{C})-4 x(\overline{C O})-x(D)+x(\bar{D}) \leq 9
\end{aligned}
$$

\section{III.3.7 Facetas das semi $k$-cercas}

Definição III.22: Um digrafo $D$ é denominado uma k-cerca se satisfaz às seguintes propriedades:

1) $|V D|=2 k, k \geq 2$;

2) Existe uma partição de $V D$ em dois subconjuntos $S=\left\{s_{1}, s_{2}, \ldots, s_{k}\right\}$ e $I=$ $\left\{i_{1}, i_{2}, \ldots, i_{k}\right\}$ tal que

$$
A D=\left\{\left(s_{j}, i_{j}\right): 1 \leq j \leq h\right\} \cup\left\{\left(i_{j}, s_{l}\right): 1 \leq j, l \leq k \text { e } j \neq l\right\}
$$


Exemplo: O digrafo abaixo é uma 4-cerca:

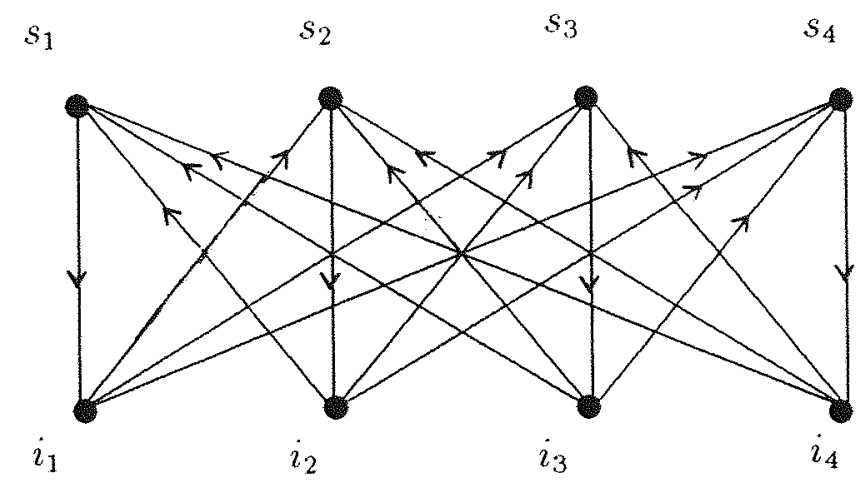

As arestas $\left(s_{j}, i_{j}\right)$ são denominadas estacas; os vértices de $S$ são denominados superiores e os de $I$, inferiores. Note que uma $k$-cerca é uma particular orientação de um grafo bipartido completo com $2 k$ vértices e que, se invertermos todas as arestas de uma $k$-cerca, obtemos novamente uma $k$-cerca.

Dada uma $k$-cerca, para obtermos um subdigrafo acíclico, precisamos retirar no mínimo $k-1$ arestas. Note que um tal conjunto minimal de arestas ou consiste de $k-1$ estacas, ou $k-2$ estacas e uma aresta que não é estaca, cujos extremos nào coincidem com os das $k-2$ estacas consideradas.

Se $D$ é uma $k$-cerca então a seguinte inequação induzida por $D$

$$
x(A D) \leq k^{2}-k+1
$$

é chamada uma inequação-k-cerca.

A inequação acima é obviamente válida para $P_{O L}(n)$ e para $P_{O P}(n), n \geq 2 k$. Reinelt [1985] mostrou que esta inequaçào é uma faceta de $P_{O L}(n)$, para todo $n \geq 2 k$. Verificamos, através de uma demonstração exaustiva que a inequação 3-cerca não é uma faceta de $P_{O P}(6)$.

É imediato que $P_{O L}(n) \subseteq P_{P O C}(n) \subseteq P_{O P}(n)$. (Na próxima seçâo mencionamos esta relação bem como outros resultados correlatos. O leitor interessado poderá consultar a próxima seção, antes de prosseguir lendo esta seçào.) 
Portanto, se $a^{T} x \leq a_{0}$ é uma inequação válida para $P_{P O C}(n)$, ela será óbviamente válida para $P_{O L}(n)$. O contrário não ocorre. Observe, por exemplo, que a inequação $k$-cerca não é válida para $P_{P O C}(n)$. Entretanto, se $a^{T} x \leq a_{0}$ é uma faceta de $P_{O L}(n)$, não necessariamente válida para $P_{P O C}(n)$, adicionando inequações válidas para $P_{P O C}(n)$ a esta inequação, eventualmente ocorre de obtermos inequações válidas (ou mesmo facetas) para $P_{P O C}(n)$. Deste modo um tanto incomum - que pode ser justificado geometricamente - foi gerada a inequação semi-k-cerca, definida a seguir.

Definição III.23: Seja $G$ um digrafo obtido de uma $k$-cerca, invertendo exatamente $k-1$ estacas, isto é:

1) $|V G|=2 k, k \geq 2$

2) $V G$ pode ser particionado em dois subconjuntos, $S=\left\{s_{1}, s_{2}, \ldots, s_{k}\right\}$ e $I=\left\{i_{1}, i_{2}\right.$, $\left.\ldots, i_{k}\right\}$ e existe um único $t, 1 \leq t \leq k$ tal que

$$
A G=\left\{\left(s_{t}, i_{t}\right)\right\} \cup\left\{\left(i_{l}, s_{j}\right): 1 \leq j, l \leq k\right\} \backslash\left\{\left(i_{t}, s_{t}\right)\right\} .
$$

Então $G$ é denominado uma semi-k-cerca.

Exemplo: O digrafo abaixo é uma semi-3-cerca.

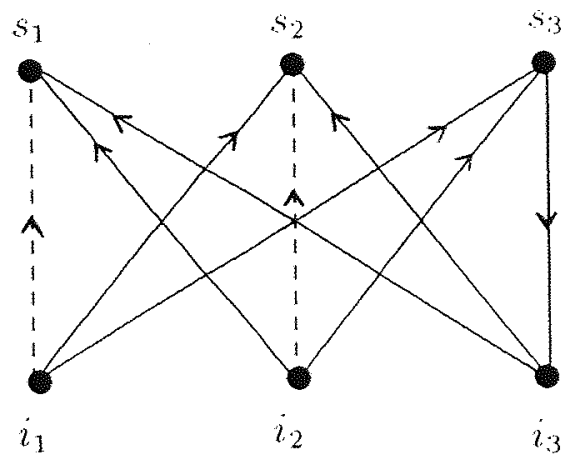

Definição III.24: Seja $G$ uma semi-k-cerca conforme a difinição IIl.23. Considere a partição de $A G$ nos conjuntos

$$
A^{-}=\left\{\left(i_{j}, s_{j}\right) \in A G: j \neq t\right\} \quad \mathrm{e}
$$




$$
A^{+}=A G \backslash A^{-} .
$$

Então a inequação induzida por $G$,

$$
x\left(A^{+}\right)-x\left(A^{-}\right) \leq k^{2}-2 k+2,
$$

é denominada inequação semi-k-cerca. Uma aresta $\alpha \in A G$ é dita positiva se $\alpha \in A^{+}$; e negativa se $\alpha \in A^{-}$.

É fácil ver que a inequação semi- $k$-cerca pode ser obtida da inequação $k$-cerca adicionando-se $k-1$ inequações de arcos paralelos, $-x_{\alpha}-x_{\bar{\alpha}} \leq-1$, uma para cada aresta $\alpha \in A^{-}$.

Teorema III.25: Seja $a^{T} x \leq a_{0}$ a seguinte inequação induzida por uma semi-k-cerca $G$, $G \subseteq D_{n}, k \geq 3$, conforme a definição III.24:

$$
x\left(A^{+}\right)-x\left(A^{-}\right) \leq k^{2}-2 k+2 .
$$

Então:

(i) $a^{T} x \leq a_{0}$ é válida para $P_{P O C}(n), n \geq 2 k$;

(ii) $a^{T} x \leq a_{0}$ é uma faceta de $P_{P O C}(n), n \geq 2 k$.

Prova: Vamos supor s.p.g., que $\left\{\left(s_{k}, i_{k}\right)\right\} \in A^{+}$. Logo, $A^{-}=\left\{\left(i_{1}, s_{1}\right),\left(i_{2}, s_{2}\right), \ldots,\left(i_{k-1}\right.\right.$, $\left.\left.s_{k-1}\right)\right\}$.

(i) A prova da validade será por simples contagem. Seja então $H$ um subdigrafo de $D_{n}$ tal que $x^{A H}$ é um vértice de $P_{P O C}(n)$ e suponha que $a^{+}=\left|A^{+} \cap A H\right|$ e $a^{-}=$ $\left|A^{-} \cap A H\right|$. Então $a^{+} \leq k^{2}-k+1$ e $a^{-} \leq k-1$. Mostraremos que

$$
x\left(A^{+} \cap A H\right)-x\left(A^{-} \cap A H\right) \leq k^{2}-2 k+2 .
$$

Temos três casos a considerar: 
Caso 1: $a^{-} \geq k-2$

Se $a^{+}=k^{2}-k+1$, isto é, $H$ contém todas as arestas positivas de $G$, segue da transitividade de $A H$ que $a^{-}=k-1$ e a desigualdade acima se verifica.

Se $a^{+} \leq k^{2}-k$, é imediato que $x\left(A^{+} \cap A H\right)-x\left(A^{-} \cap A H\right) \leq k^{2}-2 k+2$.

Caso 2: $a^{-}=k-3$

Sejam $\alpha=\left(i_{l}, s_{l}\right)$ e $\beta=\left(i_{p}, s_{p}\right)$ as duas arestas em $A^{-} \backslash A H$. Como $\bar{\alpha}$ e $\bar{\beta} \in A H$, então $\left(i_{l}, s_{p}\right) \notin A^{+}$ou $\left(i_{p}, s_{l}\right) \notin A^{+}$.

Se $\left(s_{k}, i_{k}\right) \notin A^{+}$, então $a^{+} \leq k^{2}-k-1$. Caso contrário, $\left(i_{l}, s_{k}\right) \notin A^{+}$ou $\left(i_{k}, s_{l}\right) \notin A^{+}$ e portanto $a^{+} \leq k^{2}-k-1$. Logo $a^{+}-a^{-} \leq k^{2}-2 k+2$.

Caso 3: $a^{-}<k-3$

Sejam $\alpha$ e $\beta$ duas arestas em $A^{-} \backslash A H, \alpha=\left(i_{l}, s_{l}\right)$ e $\beta=\left(i_{p}, s_{p}\right), l \neq p$. Como $x^{A H} \in P_{P O C}(n)$, então $\bar{\alpha}, \bar{\beta} \in A H$. Neste caso, ou $\left(i_{l}, s_{p}\right) \notin A H$ ou $\left(i_{p}, s_{l}\right) \notin A H ;$ caso contrário, $\alpha, \beta \in A H$, uma contradição. Concluímos aqui que para cada duas arestas negativas que não pertencem ao grafo $H$, no mínimo uma aresta positiva nâo pertence a $H$. Claramente, tais arestas positivas não pertencentes a $H$ são todas distintas. Dado que $k-1-a^{-}$arestas estão em $A^{-} \backslash A H$, no mínimo $\left(\begin{array}{c}k-1-a^{-} \\ 2\end{array}\right)$ arestas positivas não estão no grafo $H$. Portanto,

$$
a^{+} \leq k^{2}-k+1-\left(\begin{array}{c}
k-1-a^{-} \\
2
\end{array}\right) \text {. }
$$

Como $a^{-}<k-3$, então $k-1-a^{-}>2$. Isto implica em

$$
\left(\begin{array}{c}
k-1-a^{-} \\
2
\end{array}\right) \geq k-1-a^{-} .
$$

Logo, $a^{+} \leq k^{2}-k+1-\left(k-1-a^{-}\right)$, e portanto, $a^{+}-a^{-} \leq k^{2}-2 k+2$.

Isto completa a prova da validade da inequação semi-k-cerca. 
(ii) Inicialmente vamos provar que a inequação semi- $k$-cerca é uma faceta de $P_{P O C}(2 k)$. Considere $F_{b}=\left\{x \in P_{P O C}(2 k): b^{T} x=b_{0}\right\}$ uma faceta de $P_{P O C}(2 k)$ tal que $F_{b} \supseteq F_{a}=\left\{x \in P_{P O C}(2 k): a^{T} x=a_{0}\right\}$.

Vamos provar que existe $\lambda \in \mathbb{R}^{*}$ tal que $b=\lambda a$, na seguinte ordem:

a) $b_{\alpha}=0$ para todo $\alpha \in \overline{A G}$

b) $b_{\alpha}=0$ para todo $\alpha \in A D_{2 k} \backslash(A G \cup \overline{A G})$;

c) $b_{\alpha}=\lambda a_{\alpha}$ para todo $\alpha \in A G$.

a) Seja $O$ a seguinte ordem linear de $D_{2 k}$.

$$
O=\left\langle i_{1}, i_{2}, \ldots, i_{k-1}, s_{k}, i_{k}, s_{1}, s_{2}, \ldots, s_{k-1}\right\rangle
$$

Então o conjunto $A(O)$ é uma pré-ordem completa de $D_{2 k}$ tal que $x^{A(O)} \in F_{a} \subseteq$ $F_{b}$. Fica a cargo do leitor verificar que $b_{\alpha}=0$ se $\alpha \in \overline{A(O)}$.

Como $A G \subseteq A(O)$, segue que $b_{\alpha}=0$ para todo $\alpha \in \overline{A G}$.

b) Considere $S^{\prime \prime}=S \backslash\left\{s_{k}\right\} \quad$ e $\quad I^{\prime}=I \backslash\left\{i_{k}\right\}$.

Inicialmente, vamos verificar que $b_{u, v}=0$ se $u, v \in S^{\prime \prime}$.

Suponha então que $u=s_{i}$ e $v=s_{j}, 1 \leq i \neq j \leq k-1$. Considere a seguinte ordem linear de $D_{2 k}$ :

$$
O=\left\langle i_{1}, i_{2}, \ldots, i_{k-1}, s_{k}, i_{k}, s_{j}, s_{i}, O^{\prime}\right\rangle
$$

sendo $O^{\prime}$ uma seqüềncia qualquer de vértices em $S^{\prime} \backslash\left\{s_{j}, s_{i}\right\}$.

Então $A(O)$ e $A(O) \cup\left\{\left(s_{i}, s_{j}\right)\right\}$ são pré-ordens completas cujos vetores de incidência pertencem a $F_{a}$. Como $F_{a} \subseteq F_{b}$, estes vetores estão em $F_{b}$, donde:

$$
0=b^{T} x^{A(O)}-b^{T} x^{A(O) \cup\left\{\left(s_{i}, s_{j}\right)\right\}} .
$$


Portanto $b_{s_{i}, s_{j}}=0$.

Com argumento análogo, verifica-se que $b_{u, v}=0$ se $u, v \in I^{\prime}$.

Para completar a demonstração de b), devemos verificar a validade das afirmações abaixo, para todo $r \neq k$ e $l \neq k$.

$\left(\mathrm{b}_{1}\right) b_{s_{r}, s_{k}}=0$

$\left(b_{2}\right) b_{s_{k}, s_{r}}=0$

(b) $b_{i_{k}, i_{l}}=0$

(b) $b_{i_{l}, i_{k}}=0$

Como $\left(s_{k}, s_{r}\right)$ e $\left(i_{l}, i_{k}\right)$ são arestas de $A(O)$, sendo $O$ a ordem linear definida no item a), segue deste mesmo item que $b_{s_{r}, s_{k}}=b_{i_{k}, i_{l}}=0$, verificando a validade de $\left(b_{1}\right)$ e $\left(b_{3}\right)$.

Para verificar $\left(b_{2}\right)$, construa o grafo $H$, a partir do grafo $G$, invertendo as arestas $\alpha=\left(s_{k}, i_{k}\right)$ e $\beta=\left(i_{j}, s_{j}\right), j \neq r$. A aresta $\beta$ existe, pois $k \geq 3$. Então,

$$
\left|A H \cap A^{+}\right|=k^{2}-k \text { e }\left|A H \cap A^{-}\right|=k-2 .
$$

Logo,

$$
x\left(A H \cap A^{+}\right)-x\left(A H \cap A^{-}\right)=k^{2}-2 k+2 .
$$

O conjunto $A H$ pode ser estendido para a seguinte ordem linear:

$$
O=\left\langle O^{\prime}, s_{j}, i_{j}, O^{\prime \prime}, s_{r}, s_{k}\right\rangle
$$

sendo $O^{\prime}$ uma sequência de vértices em $I \backslash\left\{i_{j}\right\}$ e $O^{\prime \prime}$ uma seqüência de vértices en $S \backslash\left\{s_{j}, s_{r}, s_{k}\right\}$. Observe que $A(O)$ e $A(O) \cup\left\{\left(s_{k}, s_{r}\right)\right\}$ são préordens completas cujos vetores de incidéncia estão em $F_{a}$, e portanto, em $F_{b}$. Logo,

$$
0=b^{T} x^{A(O)}-b^{T} x^{A(O) \cup\left\{\left(s_{k}, s_{r}\right)\right\}},
$$


donde, $b_{s_{k}, s_{r}}=0$, como queríamos provar.

Com raciocínio semelhante prova-se o item $\left(\mathrm{b}_{4}\right)$. Esta demonstração fica a cargo do leitor.

c) Suponha que $b_{s_{k}, i_{k}}=\lambda, \quad \lambda \in \mathbb{R}$ e $I N D=\{1,2, \ldots, k-1\}$. Vamos provar que:

$\left(c_{1}\right) b_{i_{j}, s_{j}}=-\lambda, \quad \forall j \in I N D$

(c) $b_{i_{j}, s_{k}}=\lambda, \quad \forall j \in I N D$

(c $\left.b_{3}\right) b_{i_{k}, s_{r}}=\lambda, \quad \forall r \in I N D$

$\left(c_{4}\right) \cdot b_{i_{j}, s_{r}}=\lambda, \quad \forall r, j \in I N D, r \neq j$.

$\left(c_{1}\right)$ Considere o grafo $H$, construído a partir do grafo $G$, invertendo as arestas $\left(s_{k}, i_{k}\right)$ e $\left(i_{j}, s_{j}\right)$. Os conjuntos das arestas de $G$ e de $H$ podem ser estendidos para as ordens lineares $O_{G}$ e $O_{H}$, indicadas abaixo:

$$
\begin{aligned}
O_{G}= & \left\langle i_{1}, \ldots, i_{k-1}, s_{k}, i_{k}, s_{1}, \ldots, s_{k-1}\right\rangle \\
O_{H}= & \left\langle i_{1}, \ldots, i_{j-1}, i_{k}, i_{j+1}, \ldots, i_{k-1}, s_{j}, i_{j}, s_{1}\right. \\
& \left.\ldots, s_{j-1}, s_{k}, s_{j+1}, \ldots, s_{k-1}\right\rangle
\end{aligned}
$$

Então $A\left(O_{G}\right)$ e $A\left(O_{H}\right)$ são pré-ordens completas e $x^{A\left(O_{G}\right)}, x^{A\left(O_{H}\right)} \in F_{a} \subseteq$ $F_{b}$. Usando os resultados obtidos nos itens a) e b), concluímos que

$$
\begin{aligned}
& b_{i_{j}, s_{j}}+b_{s_{k}, i_{k}}=0, \text { donde } \\
& b_{i_{j}, s_{j}}=-b_{s_{k}, i_{k}} .
\end{aligned}
$$

Logo, $b_{i_{j}, s_{j}}=-\lambda$.

$\left(c_{2}\right)$ Considere o grafo $H$, construído a partir do grafo $G$, invertendo as arestas $\left(i_{j}, s_{k}\right)$ e $\left(i_{j}, s_{j}\right)$. Como no caso $\left(c_{1}\right)$, considere a ordem linear $O_{H}$ 
estendendo o conjunto das arestas de $H$ :

$$
\begin{aligned}
O_{H}= & <i_{1}, \ldots, i_{j-1}, i_{j+1}, \ldots, i_{k-1}, s_{k}, i_{k}, s_{j}, i_{j}, s_{1}, \\
& \ldots, s_{j-1}, s_{j+1}, \ldots, s_{k-1}>.
\end{aligned}
$$

Não é difícil ver que $x^{A\left(O_{H}\right)} \in F_{a} \subseteq F_{b}$. Então

$$
b^{T} x^{A\left(O_{H}\right)}-b^{T} x^{A\left(O_{G}\right)}=0,
$$

sendo $O_{G}$ a ordem linear definida em $\left(c_{1}\right)$.

Usando resultados provados anteriormente, concluímos que

$$
b_{i_{j}, s_{k}}+b_{i_{j}, s_{j}}=0 .
$$

De $\left(c_{1}\right)$, segue que $b_{i, s_{k}}=\lambda$.

$\left(c_{3}\right)$ Considere agora o grafo $H$, obtido a partir de $G$, invertendo as arestas $\left(i_{k}, s_{r}\right)$ e $\left(i_{r}, s_{r}\right)$. Como em $\left(c_{2}\right)$, temos a ordem linear

$$
\begin{aligned}
O_{H}= & \left\langle i_{1}, \ldots, i_{r-1}, i_{r+1}, \ldots, i_{k-1}, s_{r}, i_{r}, s_{k}, i_{k}, s_{1},\right. \\
& \left.\ldots, s_{r-1}, s_{r+1}, \ldots, s_{k-1}\right\rangle
\end{aligned}
$$

Analogamente ao caso anterior, obtemos

$$
b_{i_{r}, s_{r}}+b_{i_{k}, s_{r}}=0 .
$$

De $\left(c_{1}\right)$, segue que $b_{i_{k}, s_{r}}=\lambda$.

(c) Suponha, s.p.g., que $r<j$ e considere o grafo $H$, construido a partir do grafo $G$, invertendo as arestas $\left(s_{k}, i_{k}\right),\left(i_{j}, s_{r}\right),\left(i_{j}, s_{j}\right)$ e $\left(i_{r}, s_{r}\right)$. O conjunto 
das arestas de $H$ pode ser estendido para a seguinte ordem linear:

$$
\begin{aligned}
O_{H}= & \left\langle i_{1}, \ldots, i_{r-1}, i_{r+1}, \ldots, i_{j-1}, i_{j+1}, \ldots, i_{k}, s_{r}, i_{r}, s_{j}, i_{j}, s_{1},\right. \\
& \left.\ldots, s_{r-1}, s_{r+1}, \ldots, s_{j-1}, s_{j+1}, \ldots, s_{k}\right\rangle .
\end{aligned}
$$

Observe que $A\left(O_{H}\right)$ é uma pré-ordem completa e que as únicas arestas positivas de $G$ que não estão em $A\left(O_{H}\right)$ são as arestas $\left(i_{j}, s_{r}\right)$ e $\left(s_{k}, i_{k}\right)$. Como as únicas arestas negativas de $G$ que não pertencem a $A\left(O_{H}\right)$ são as arestas $\left(i_{j}, s_{j}\right)$ e $\left(i_{r}, s_{r}\right)$, segue que

$$
x\left(A\left(O_{H}\right) \cap A^{+}\right)+x\left(A\left(O_{H}\right) \cap A^{-}\right)=k^{2}-2 k+2
$$

e portanto, $x^{A\left(O_{H}\right)} \in F_{a}$. Como $F_{a l} \subseteq F_{b}$, vem que

$$
b^{T} x^{A\left(O_{G}\right)}-b^{T} x^{A\left(O_{H}\right)}=0,
$$

sendo $O_{G}$ a ordem linear definida em $\left(c_{1}\right)$.

Usando os resultados obtidos em a) e b), podemos concluir que

$$
b_{s_{k}, i_{k}}+b_{i_{r}, s_{r}}+b_{i_{j}, s_{j}}+b_{i_{,}, s_{r}}=0 .
$$

Como $b_{i_{r}, s_{r}}=b_{i_{j}, s_{j}}=-\lambda$ por $\left(c_{1}\right)$ e $b_{s_{k}, i_{k}}=\lambda$, por hipótese, segue que $b_{i_{j}, s_{r}}=\lambda$, o que completa a prova deste item.

Os itens $\left(c_{1}\right),\left(c_{2}\right),\left(c_{3}\right)$ e $\left(c_{4}\right)$ provam que

$$
b_{\alpha}=\lambda a_{\alpha} \quad \text { se } \alpha \in A G, \text { sendo } \lambda \in \mathbb{R} .
$$

De a), b) e c), concluímos que

$$
b=\lambda a \quad \text { para } \lambda \in \mathbb{R}^{*} .
$$

Logo, $a^{T} \leq a_{0}$ é uma faceta de PPOC $(2 k)$. 
Como a condição do lema da elevação é facilmente verificada para esta inequação, segue que

$$
x\left(A^{+}\right)-x\left(A^{-}\right) \leq k^{2}-2 k+2
$$

é uma faceta de $P_{P O C}(n), n \geq 2 k$, como queríamos demonstrar.

Note que para $n \geq 6$ a classe das inequações semi- $k$-cerca, $3 \leq k \leq\left\lfloor\frac{n}{2}\right\rfloor$, é bastante grande. A cardinalidade desta classe é:

$$
\sum_{k=3}^{\left\lfloor\frac{n}{2}\right\rfloor}\left[\left(\begin{array}{c}
n \\
2 k
\end{array}\right)\left(\begin{array}{c}
2 k \\
k
\end{array}\right) k\right]
$$

\section{III.4 Relação entre os politopos}

Nesta seção apresentamos a relação com respeito à inclusão entre os politopos $P_{O L}(n)$, $P_{P O C}(n), P_{O P}(n)$ e $P_{P O}(n)$. Além disso, veremos quais dentre esses politopos são faces de outros. Para isto, observemos inicialmente que:

$$
\begin{aligned}
& P_{O L}(n)=\operatorname{conv}\left\{\begin{array}{ll}
x \in\{0,1\}^{A_{n}}: x_{i j}+x_{j i}=1, \quad i, j \in V_{n}, \quad i \neq j \quad \text { e } \\
& x_{i j}+x_{j k}-x_{i k} \leq 1, \quad i, j, k \in V_{n}, \quad i \neq j \neq k \neq i
\end{array}\right\} \\
& P_{P O C}(n)=\operatorname{conv}\left\{\begin{array}{ll}
x \in\{0,1\}^{A_{n}} \quad & x_{i j}+x_{j i} \geq 1, \quad i, j \in V_{n}, \quad i \neq j \quad \text { e } \\
& x_{i j}+x_{j k}-x_{i k} \leq 1, \quad i, j, k \in V_{n}, \quad i \neq j \neq k \neq i
\end{array}\right\} \\
& P_{O P}(n)=\operatorname{conv}\left\{\begin{aligned}
& x \in\{0,1\}^{A_{n}}: x_{i j}+x_{j i} \leq 1, \quad i, j \in V_{n}, \quad i \neq j \quad \text { e } \\
& x_{i j}+x_{j k}-x_{i k} \leq 1, \quad i, j, k \in V_{n}, \quad i \neq j \neq k \neq i
\end{aligned}\right\} \\
& P_{P O}(n)=\operatorname{conv}\left\{x \in\{0,1\}^{A_{n}}: x_{i j}+x_{j k}-x_{i k} \leq 1, \quad i, j, k \in V_{n}, \quad i \neq j \neq k \neq i\right\} .
\end{aligned}
$$


É imediato que a seguinte relação de inclusão ocorre entre esses politopos:

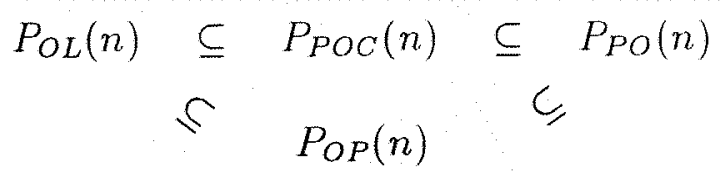

Também é fácil ver que para $n \geq 3$ todas as inclusôes indicadas são próprias.

Uma outra relação que pode ser facilmente verificada é:

$$
P_{O L}(n)=P_{O P}(n) \cap P_{P O C}(n)
$$

Convém aqui observar que

$$
P_{P O}(n) \supseteq P_{O P}(n) \cup P_{P O C}(n)
$$

e que esta inclusão é própria para $n \geq 3$.

Mais forte do que a simples relação de inclusão entre $P_{O L}(n)$ e $P_{P O C}(n)$ é o seguinte fato:

$$
P_{O L}(n) \text { é uma face de } P_{P O C}(n) \text {. }
$$

Para verificar isto, para cada aresta $\alpha \in A_{n}$, considere o conjunto

$$
F_{\alpha}=\left\{x \in P_{P O C}(n): x_{\alpha}+x_{\overline{\alpha x}}=1\right\} .
$$

É imediato que

$$
P_{O L}(n)=\bigcap_{\alpha \in A_{n}} F_{\alpha} .
$$

De acordo com o teorema III.7, $F_{\alpha}$ é uma faceta de $P_{P O C}(n)$. Portanto, $P_{O L}(n)$ é uma face de $P_{P O C}(n)$. Como $F_{\alpha}$ é também uma faceta de $P_{O P}(n)$, conforme o teorema 111.7, analogamente, podemos concluir que

$$
P_{O L}(n) \text { é uma face de } P_{O P}(n) \text {. }
$$

Além disso, é fácil verificar que

$$
\operatorname{dim} P_{O L}(n)=\frac{n(n-1)}{2}
$$

No caso $n=2$ a relação entre os politopos pode ser visualizada na figura abaixo: 


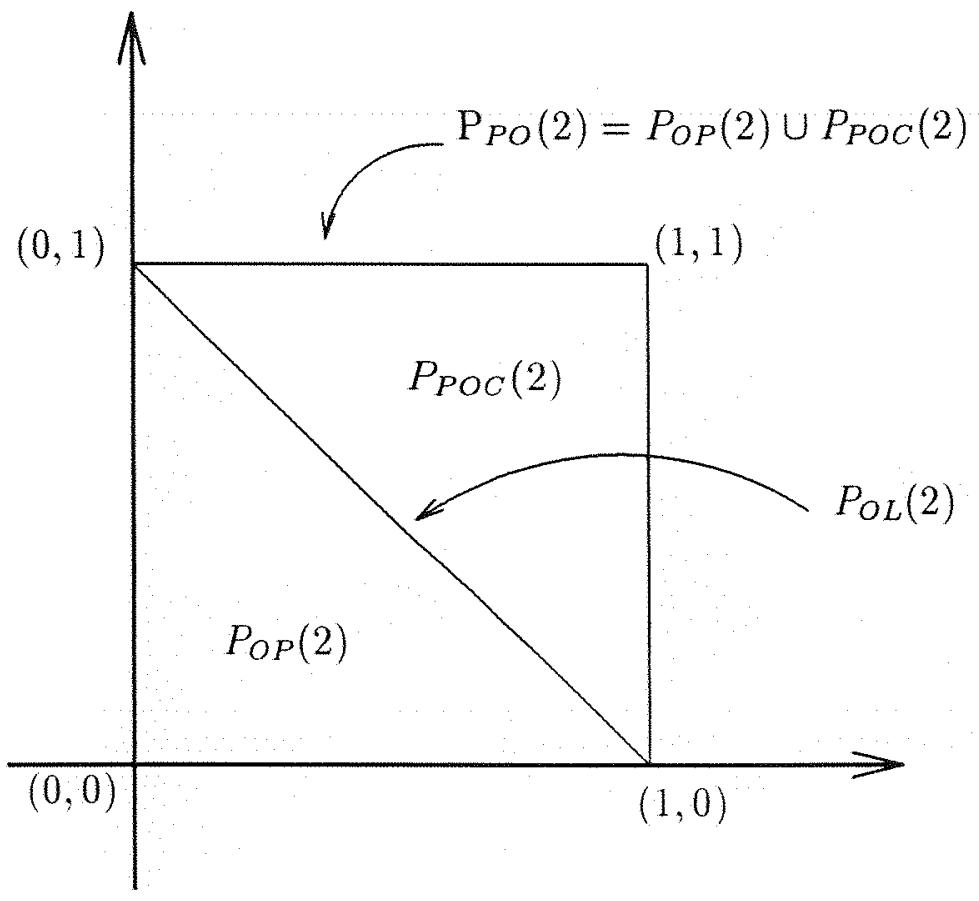

Note que $P_{O P}(2)$ e $P_{P O C}(2)$ não são faces de $P_{P O}(2)$.

\section{III.5 Soluções fracionárias}

Nesta seçâo mostraremos que os politopos obtidos através de relaxaçòes simples dos politopos em questão possuem vértices fracionários. Faremos a análise para o caso $n=$ 5 , mas resultados análogos podem ser obtidos quando $n>5$. Considere as seguintes inequações/restrições em $\mathbb{R}^{A_{5}}$ :

(1) $x_{\alpha}+x_{\bar{\alpha}} \geq 1 \quad \forall \alpha \in A_{5}$,

(2) $x_{\alpha}+x_{\bar{\alpha}} \leq 1 \quad \forall \alpha \in A_{5}$,

(3) $x_{i j}+x_{j k}-x_{i k} \leq 1 \quad \forall i, j, k \in V_{5}, \quad i \neq j \neq k \neq i$,

(4) $x_{\alpha} \in\{0,1\} \quad \forall \alpha \in A_{5}$,

(5) $0 \leq x_{\alpha} \leq 1 \quad \forall \alpha \in A_{5}$. 


\section{Note que}

$$
\begin{aligned}
& P_{P O C}(5)=\operatorname{conv}\left\{x \in \mathbb{R}^{A_{5}}: x \operatorname{satisfaz}(1),(3) \mathrm{e}(4)\right\} \\
& P_{O P}(5)=\operatorname{conv}\left\{x \in \mathbb{R}^{A_{5}}: x \operatorname{satisfaz}(2),(3) \mathrm{e}(4)\right\} \mathrm{e} \\
& P_{P O}(5)=\operatorname{conv}\left\{x \in \mathbb{R}^{A_{5}}: x \operatorname{satisfaz}(3) \mathrm{e}(4)\right\}
\end{aligned}
$$

Chamemos de $\hat{P}_{P O C}(5), \hat{P}_{O P}(5)$ e $\hat{P}_{P O}(5)$ os politopos obtidos de $P_{P O C}(5), P_{O P}(5)$ e $P_{P O}(5)$, respectivamente, substituindo-se a restrição (4) pela restrição (5). Note que os conjuntos de pontos que satisfazem as respectivas restriçòes são convexos e limitados e portanto,

$$
\begin{aligned}
& \hat{P}_{P O C}(5)=\left\{x \in \mathbb{R}^{A_{5}}: x \operatorname{satisfaz}(1),(3) \mathrm{e}(5)\right\} \\
& \hat{P}_{O P}(5)=\left\{x \in \mathbb{R}^{A_{5}}: x \operatorname{satisfaz}(2),(3) \mathrm{e}(5)\right\} \text { e } \\
& \hat{P}_{O P}(5)=\left\{x \in \mathbb{R}^{A_{5}}: x \operatorname{satisfaz}(3) \mathrm{e}(5)\right\}
\end{aligned}
$$

Claramente, $P_{P O C}(5) \subseteq \hat{P}_{P O C}(5), P_{O P}(5) \subseteq \hat{P}_{O P}(5)$ e $P_{P O}(5) \subseteq \hat{P}_{P O}(5)$. Vamos mostrar que estas inclusões são todas próprias, ou seja, que estes novos politopos possuem vértices fracionários. Para isto, vamos exibir primeiramente uma função peso $p: A_{5} \rightarrow \mathbb{Z}$ tal que

$$
\begin{gathered}
\max \left\{p^{T} x: x \in \hat{P}_{P O}(5)\right\} \quad \mathrm{e} \\
\max \left\{p^{T} x: x \in \hat{P}_{O P}(5)\right\}
\end{gathered}
$$

têm soluções ótimas fracionárias que são vértices do respectivo politopo.

Considere um circuito hamiltoniano $C=\langle 1,2,3,4,5\rangle$ em $D_{5}$, e defina $p^{T} x$ como

$$
p^{T} x=x(C)-x(C O)
$$

sendo $C O$ o conjunto das 2-cordas de $C$. 
O vetor $\widehat{x} \in \mathbb{R}^{5}$ tal que

$$
\widehat{x}_{\alpha}= \begin{cases}1 / 2, & \text { se } \alpha \in C \\ 0, & \text { caso contrário }\end{cases}
$$

é um vértice de $\widehat{P}_{P O}(5)$ e de $\widehat{P}_{O P}(5)$ que é uma solução ótima de (6) e de (7), ambos com valor objetivo ótimo $=5 / 2$.

Note que $\hat{x}$ não satisfaz a restrição

$$
x(C)-x(C O) \leq 2
$$

que é uma inequação-faceta de $P_{P O}(5)$ e de $P_{O P}(5)$, conforme o teorema III.11. A título de comentário, vale observar que, após acrescentar está inequação ao sistema que define $\widehat{P}_{O P}(5)$ (resp. $\left.\widehat{P}_{P O}(5)\right)$, obtivemos uma solução ótima $0 / 1$.

Vamos agora mostrar que $\hat{P}_{P O C}(5)$ tem vértice fracionário. Para isto, considere a função peso $\bar{p}: A_{5} \rightarrow \mathbb{Z}$ como sendo

$$
\bar{p}^{T} x=3 x(C)-3 x(C O)+x(\bar{C})-x(\overline{C O})
$$

Resolvendo-se o PL

$$
\max \left\{\bar{p}^{T} x: x \in \hat{P}(5)\right\}
$$

usando o método simplex, obtivemos como soluçào ótima o vetor $\bar{x}$ (que é portanto um vértice de $\left.\hat{P}_{P O C}(5)\right)$ cujos componentes têm os valores indicados no digrafo $G$ abaixo, sendo $\bar{x}_{\alpha}=0$ se $\alpha \notin A G$. 


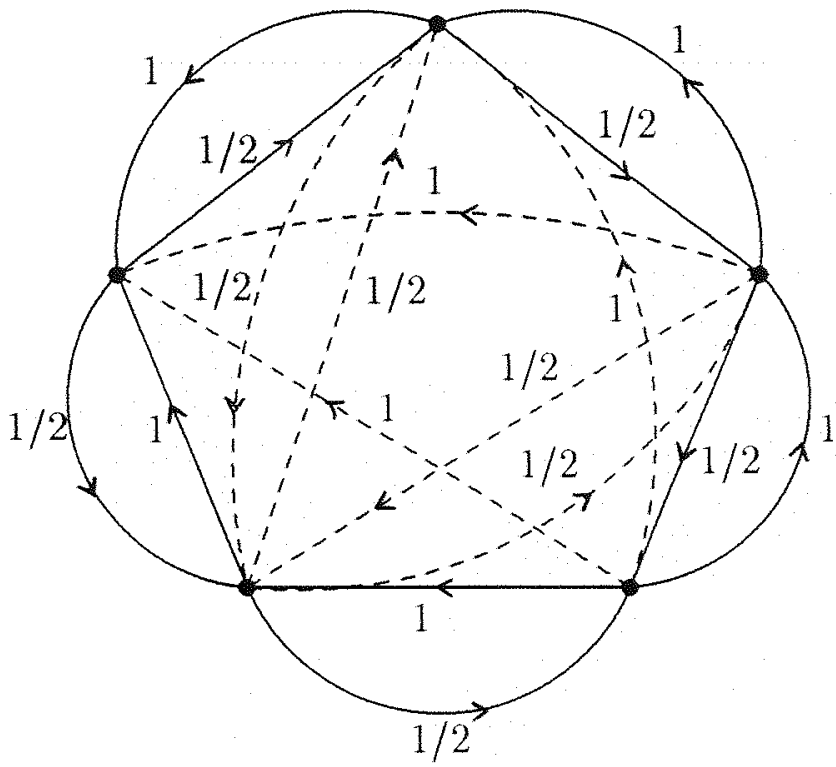

Podemos verificar que $\bar{p}^{T} \bar{x}=11 / 2$, e portanto, $\bar{x}$ nào satisfaz a restriçào

$$
3 x(C)-3 x(C O)+x(\bar{C})-x(\overline{C O}) \leq 4
$$

que é uma inequação-faceta de $P_{P O C}(5)$, de acordo com o teorema III.16.

Acrescentamos esta última inequação ao sistema que define $\widehat{P}_{P O C}(5)$ e resolvemos o novo LP, obtendo desta vez a soluçào ótima $x^{*}$, indicada no digrafo $C^{*}$ a seguir, sendo $x_{\alpha}^{*}=0$ se $\alpha \notin A G^{*}$ e $x_{\alpha}^{*}=1$, se $\alpha \in A G^{*}$.

Como $x^{*}$ é uma solução $0 / 1$ e pertence ao politopo $\operatorname{Ppoc}(5)$, então $x^{*}$ é o vetor característico de uma pré-ordem completa de $P_{P O C}(5)$. Logo, $x^{*}$ é também solução ótima da seguinte instância:

$$
\max \left\{\bar{p}^{T} x: x \in P_{P O C}(5)\right\}
$$




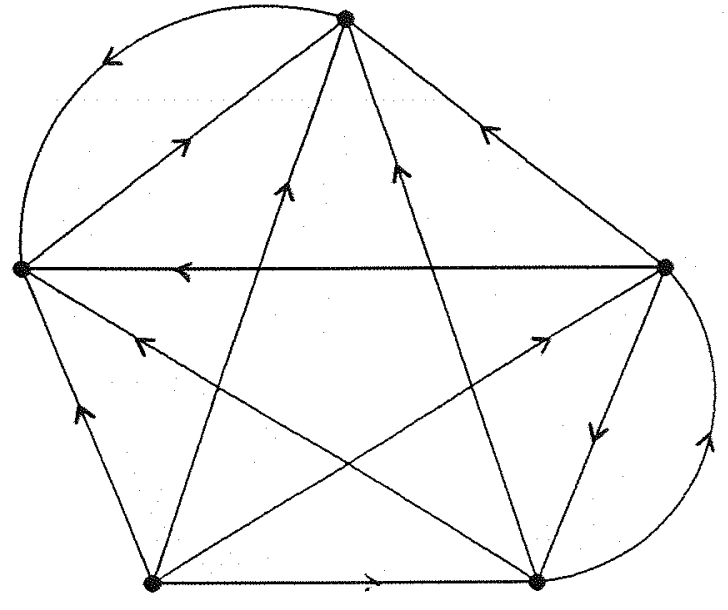




\section{Capítulo IV}

\section{Diâmetro e adjacência de vértices do politopo da pré-ordem completa}

Quando duas pré-ordens completas, digamos $S$ e $T$, estão relacionadas pela inclusão, o digrafo induzido pela diferença simétrica de $S$ e $T$ tem uma estrutura simples, que explorada convenientemente possibilitou-nos a determinação do diâmetro de $P_{P O C}(n)$.

É imediato que diam $\left(P_{P O C}(2)\right)=1$. Provamos neste capítulo que diam $\left(P_{P O C}(n)\right)=$ 2 , se $n \geq 3$. O fato de que este diâmetro é pequeno nos surpreendeu, apesar de encontrarmos na literatura vários exemplos de problemas INIP-difíceis, cujos politopos associados possuem diâmetros pequenos. Isto ocorre, por exemplo, no politopo do caixeiro viajante e no da ordem linear, cujos diâmetros têm valor 2 (Padberg e Rao [1974] e Young [1978]).

Infelizmente, não conseguimos caracterizar adjacência de dois vértices quaisquer de $P_{P O C}(n)$; assim, apresentamos apenas alguns resultados que caracterizam adjacência de 
vértices particulares. Cabe aqui observar, entretanto, que embora em alguns casos, como no politopo da ordem linear, adjacência de dois vértices quaisquer pode ser facilmente testada, no politopo do caixeiro viajante, decidir se dois vértices são não adjacentes é INIP-completo (Papadimitriou [1978]).

Neste capítulo, a notação p.o.c. será usada para abreviar o termo pré-ordem completa. Consideraremos $n \geq 2$, sempre que o valor de $n$ não estiver explícito.

Seja $T$ uma p.o.c. de $D_{n}$. Então os subgrafos maximais fortemente conexos de $[T]$ são cliques (maximais). Além disso, se contrairmos cada um destes cliques, obtemos o grafo condensado de $[T]$, cujas arestas constituem uma ordem linear. Esta ordem pode ser representada pela seqüência de vértices que a define. Assim, denotamos por $\quad O L T=$ $\left\langle T_{1}, \ldots, T_{k}\right\rangle$ tal seqüência, sendo $T_{1}, \ldots, T_{k}$ os cliques maximais de $[T]$. Portanto, $T$ estará bem definida, se especificada a ordem linear sobre os cliques maximais de $[T]$.

Uma aresta $\alpha \in T$ é denominada local em $T$ se seus extremos estào num mesmo clique de $O L T$; caso contrário, é denominada de ligação em $T$. Note que se $\alpha=(u, v)$ é uma aresta de ligação em $T$ tal que $u \in V T_{i}$ e $v \in V T_{j}$, então $i<j$.

Sejam $S$ e $T$ pré-ordens completas de $D_{n}$. Dizemos que $S$ é um refinamento de $T$ se e somente se $S \subseteq T$. O exemplo abaixo ilustra duas p.o.c. $S$ e $T$ em $D_{10}$ tais que $S \subseteq T$.

Exemplo IV.1: Sejam $S$ e $T$ p.o.c. em $D_{10}$, onde $V D_{10}=\{1,2, \ldots, 10\}$, definidos por:

$$
\begin{aligned}
O L S & =\langle[\{1,2\}],[\{3,5\}],[\{4,6\}],[\{7\}],[\{8,9\}],[\{10\}]>\mathrm{e} \\
O L T & =\langle[\{1,2\}],[\{3,4,5,6\}],[\{7\}],[\{8,9,10\}]\rangle .
\end{aligned}
$$

Na figura IV.1, ilustramos as pré-ordens completas $S$ e $T$, enfatizando os cliques de OLS e OLT. Omitimos as arestas entre cliques nào consecutivos. implicadas pela transitividade. 
$S:$

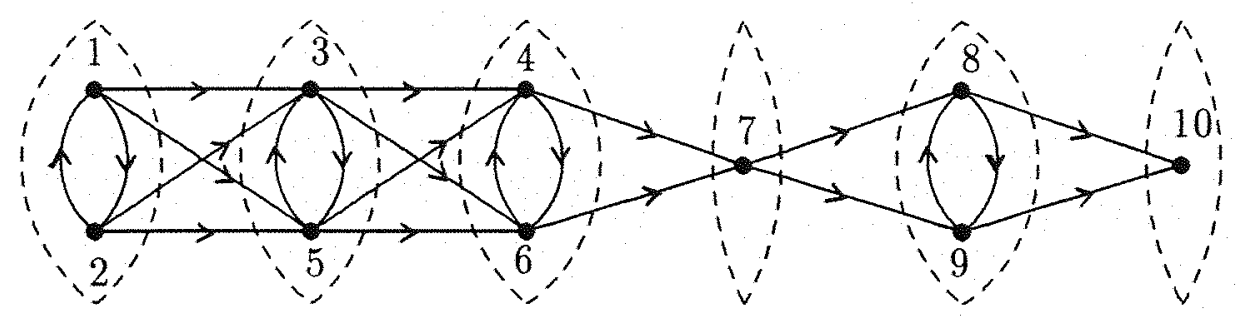

$T:$

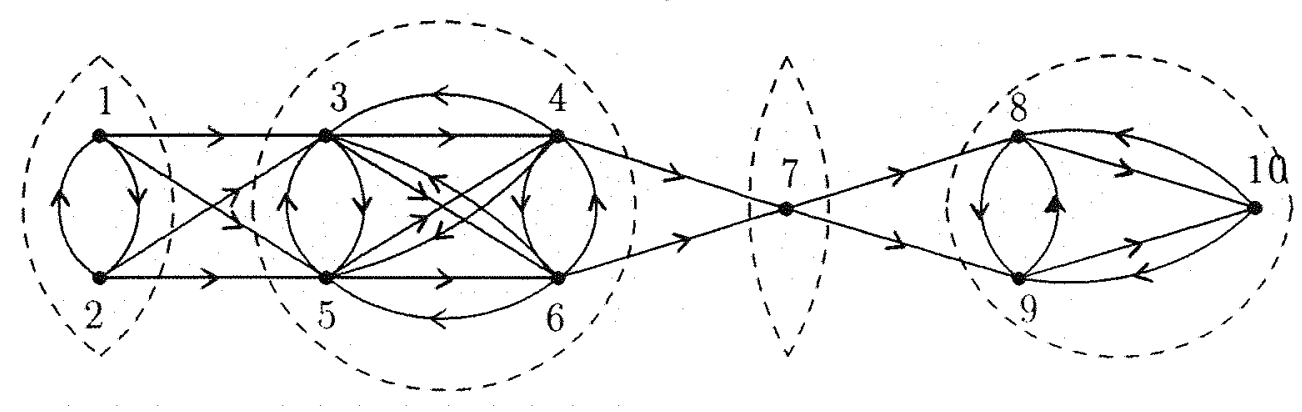

FIGURA IV.1 
Se $S$ e $T$ são p.o.c. tais que $S$ é um refinamento de $T$, então todas as arestas de ligação em $T$ são arestas de ligação em $S$ e todas as arestas locais em $S$ são locais em $T$. Usando este fato, fica fácil verificar a validade da seguinte proposição:

Proposição IV.2: Sejam $S$ e $T$ p.o.c. em $D_{n}$ com $O L S=<S_{1}, \ldots, S_{p}>$ e $O L T=$ $<T_{1}, \ldots, T_{q}>$. Se $S \subseteq T$, então para todo $i, j$ tais que $1 \leq i<j \leq p$, são válidas:

(a) $S_{i} \subseteq T_{\ell}$, para algum $\ell, 1 \leq \ell \leq q$.

(b) Se $S_{i}, S_{j} \subseteq T_{\ell}(1 \leq \ell \leq q)$, então $S_{k} \subseteq T_{\ell}$ para todo $k, i \leq k \leq j$.

Prova: A validade de (a) é imediata. Para provar (b), suponha por contradição que existam $S_{i}, S_{j} \subseteq T_{\ell}$ e $S_{k} \subseteq T_{m}$, sendo $i<k<j$ e $m \neq \ell$. Sejam $u, v$ e $w$ vértices em $V S_{i}, V S_{k}$ e $V S_{j}$, respectivamente. De $(u, v) \in S$, segue que $(u, v) \in T$. Como $u \in V T_{\ell}$ e $v \in V T_{m}$, a aresta $(u, v)$ é de ligação em $T ; \log o, \ell<m$. Por outro lado, como $(v, w) \in S$, por um raciocínio análogo conclui-se que $(v, w)$ é uma aresta de ligação em $T$ e que $m<\ell$, uma contradição.

O corolário abaixo pode ser facilmente provado:

Corolário IV.3: Sejam $S$ e $T$ como na proposição IV.Q, sendo $S \subseteq T$. Entâo os vértices de cada clique de OLT sấo vértices de cliques consecutivos de OLS. Mais precisamente, OLS pode ser expresso na forma:

$$
O L S=<S_{1}=\underbrace{S_{1}^{1}, \ldots, S_{t_{1}}^{1}}, \underbrace{S_{1}^{2}, \ldots, S_{t_{2}}^{2}}, \ldots, \underbrace{S_{1}^{q}, \ldots, S_{t_{q}}^{q}=S_{p}}>,
$$

onde $V S_{1}^{i} \cup V S_{2}^{i} \cup \cdots \cup V S_{t_{i}}^{i}=V T_{i}, \quad$ para $i=1, \ldots, q$.

As próximas proposições são relativas ao digrafo $H$, gerado por $T \backslash S$, onde $S$ e $T$ são p.o.c. tais que $S \subseteq T$. O resultado que apresentaremos na proposição IV.4 não será usado 
posteriormente, mas é interessante por si só. Na figura IV.2, ilustramos o digrafo $[T \backslash S]$, para $S$ e $T$ dados na figura IV.1.
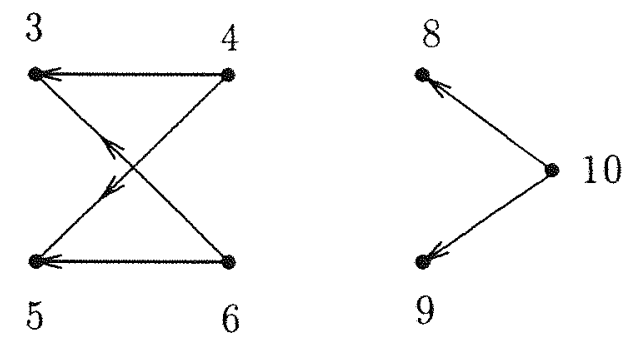

FIGURA IV.2

Proposição IV.4: Sejam $S$ e T p.o.c. distintas de $D_{n}$ tais que $S \subseteq T, O L S=$ $<S_{1}, \ldots, S_{p}>$ e OLT $=<T_{1}, \ldots, T_{q}>$. Então o digrafo gerado por $T \backslash S$ é acíclico transitivo.

Prova: Seja $H=[T \backslash S]$. É imediato que $H$ não possui circuitos dirigidos, pois se $(u, v) \in A H$, então $u \in V S_{i}, v \in V S_{j}$, sendo $i>j$.

Para mostrar que $H$ é um digrafo transitivo, suponha que $(u, v)$ e $(v, w)$ sâo arestas de $H$. Como $S$ tem a propriedade total e $(u, v) \notin S$, segue que $(v, u) \in S$. Neste caso, se $(u, w)$ pertencesse a $S$, então da transitividade de $S$ teríamos que $(v, w) \in S$, uma contradição. $\operatorname{Logo},(u, w) \notin S$. Por outro lado, como $(u, v)$ e $(v, w) \in T$, segue que $(u, w) \in T$, da transitividade de $T$. Logo, $(u, w) \in T \backslash S$, e portanto é uma aresta de $H$.

Proposição IV.5: Sejam $S$ e T p.o.c de $D_{n}$ e $H=[T \backslash S]$, com componentes $H_{1}, \ldots, H_{p}$, $p \geq 2$. Se $S \subseteq T$ e $O L T=<T_{1}, \ldots, T_{q}>$, entâa para todo $i, j, 1 \leq i<j \leq p$, sâa válidas as seguintes afirmações: 
(a) $H_{i} \subseteq T_{l}$, para algum $l, 1 \leq l \leq q$.

(b) Se $H_{i} \subseteq T_{l}$ e $H_{j} \subseteq T_{m}(1 \leq l, m \leq q)$, entấo $l \neq m$.

Prova: A validade de (a) é imediata, uma vez que $H_{i}$ tem pelo menos uma aresta, é conexo e só contém arestas locais em $T$. Para provar (b), suponha por contradição que $H_{i}, H_{j} \subseteq T_{l}$, para algum $l, 1 \leq l \leq q$. Como $H$ é gerado por um conjunto de arestas, $H$ não possui vértices isolados. Seja $\alpha=(u, v) \in A H_{i}$ e $w \in V H_{j}$. Como $(u, w)$ e $(w, v)$ são arestas de $T \backslash A H$, então estas arestas estão em $S$. Da transitividade de $S$, segue que $\alpha \in S$, uma contradição.

O próximo resultado mostra que existe uma relação especial entre os cliques de OLT e $O L S$, quando $H=[T \backslash S]$ é conexo. Como veremos, a existência de tal relação também garante a conexidade de $H$.

Corolário IV.6: Sejam $S$ e $T$ p.o.c. distintas em $D_{n}$ tais que $S \subseteq T$ e seja $H=[T \backslash S]$. Então $H$ é conexo se e somente se existe um único clique em OLT cujo conjunto de vértices é a união dos conjuntos de vértices de dois ou mais cliques (consecutivos) de $O L S$.

Prova: A demonstração deste resultado é imediata, a partir do Corolário IV.3 e da Proposição IV.5.

Teorema IV.7: Sejam $S$ e $T$ p.o.c. distintas de $D_{n}$ tais que $S \subseteq T$ e seja $H=[T \backslash S]$. Então $x^{S}$ é adjacente a $x^{T}$ em PPOC $(n)$ se e somente se $H$ é conexo.

Prova: Suponha inicialmente que $H$ é conexo. Da Proposição IV.5, segue que $H \subseteq T_{i}$, para algum clique $T_{i}$ de $O L T$. Suponha, s.p.g., que $H \subseteq T_{1}$. Do Corolário IV.6 concluímos 
que $T_{1}$ é o único clique de $O L T$ cujo conjunto de vértices é a união de conjuntos de vértices de dois ou mais cliques de OLS. Pelo Corolário IV.3 podemos então supor que

$$
O L T=<T_{1}, \ldots, T_{k}>\quad \text { e } O L S=<S_{1}, \ldots, S_{m}, \ldots, S_{m+k-1}>
$$

sendo $\quad V T_{1}=\bigcup_{i=1}^{m} V S_{i}, m \geq 2, \quad$ e $\quad V T_{j}=V S_{m+j-1}, \quad 2 \leq j \leq k$.

Seja $\alpha=(w, z) \in A H, \operatorname{com} w \in V S_{m}$ e $z \in V S_{1}$. Denote por $t$ o número de arestas de $A T_{1} \backslash S$ e considere a função $p: A D_{n} \rightarrow \mathbb{Z}$ definida por:

$$
p(\beta)=\left\{\begin{aligned}
t-1 & , \text { se } \beta=(w, z) \\
-1 & , \text { se } \beta \in A T_{1} \backslash S, \quad \beta \neq(w, z) \\
t & , \text { se } \beta \in S \\
-t & , \text { se } \beta \in A D_{n} \backslash T .
\end{aligned}\right.
$$

Então $p(T)=p(S)+p\left(A T_{1} \backslash S\right)=t|S|$, e portanto,

$$
p(T)=p(S)=t|S|
$$

Seja $R$ uma p.o.c. de $D_{n}$ cujo vetor característico maximiza a função $\sum_{\ell \in A D_{n}} p_{\ell} x_{\ell}$. Da igualdade (1), segue que

$$
\sum_{\ell \in A D_{n}} p_{\ell} x_{\ell}^{K} \geq t|S|
$$

Como $p(\beta)=t$ para toda aresta $\beta \in S$ e, excetuando a aresta $(w, z)$, todas as demais arestas têm peso negativo, de (2) conclúmos que $S \subseteq R$ e que se $p(\beta)=-t$, então $\beta \notin R$.

Se $(w, z) \notin R$, concluímos de (2) que $\beta \notin R$ para toda aresta $\beta \in A T_{1} \backslash S$. Neste caso, temos que $R=S$. Se $(w, z) \in R$, da transitividade de $R$ fica fácil ver que $\beta \in R$ para toda aresta $\beta \in A T_{1}$. Neste caso, $R=T$.

Portanto, $x^{T}$ e $x^{S}$ sào os únicos vértices de $P_{P O C}(n)$ que maximizam a função acima. Logo, pelo Teorema 1.5 , são adjacentes neste politopo. 
Para provar que a conexidade de $H$ é condição necessária, suponha por contradição que $H$ possui componentes $H_{1}, \ldots, H_{p}, p \geq 2$, e considere os subconjuntos de $A D_{n}$ definidos por:

$$
Q_{i}=S \cup A H_{i}, \quad i=1, \ldots, p
$$

Da Proposição IV.5, segue que existe $T_{\ell} \in O L T$ tal que $H_{1} \subseteq T_{\ell}$. Suponha, s.p.g., que $\ell=1$. Não é difícil verificar que $Q_{1}$ é uma p.o.c. de $D_{n}$ tal que:

$$
\begin{aligned}
O L Q_{1} & =\left\langle T_{1}, S_{t}, S_{t+1}, \ldots, S_{k}\right\rangle, \quad \text { sendo } \\
O L S & =\left\langle S_{1}, \ldots, S_{t}, \ldots, S_{k}\right\rangle, \quad \text { e } \quad V T_{1}=\bigcup_{j=1}^{t-1} V S_{j} .
\end{aligned}
$$

Com raciocínio análogo, verifica-se que cada $Q_{i}, i=2, \ldots, p$, é uma p.o.c. de $D_{n}$. Logo, $x^{Q_{i}}$ é vértice de $P_{P O C}(n)$, para $i=1, \ldots, p$.

Considere agora um ponto $y_{\lambda}$ no interior do segmento que liga $x^{T}$ a $x^{\mathcal{S}}$ em $P_{P O C}(n)$, i.e.,

$$
y_{\lambda}=\lambda x^{S}+(1-\lambda) x^{T}, \quad \text { onde } 0<\lambda<1 .
$$

Note que, como $S \subseteq T$, então $y_{\lambda}=x^{S}+(1-\lambda) x^{T \backslash S}$. Por outro lado, usando os resultados anteriores, temos que

$$
y_{\lambda}=(1-p+p \lambda) x^{S}+\sum_{i=1}^{p}(1-\lambda) x^{Q_{2}} .
$$

Logo, tomando-se $\lambda$ tal que $\frac{p-1}{p}<\lambda<1$, segue que $y_{\lambda}$ pode ser escrito de duas formas distintas como combinação convexa de vértices de $P_{P O C}(n)$. Pelo Teorema $1.5, x^{S}$ não é adjacente a $x^{T}$ neste politopo, uma contradição. 
À primeira vista, pareceu-nos que $[S \Delta T]$ conexo fosse uma condição necessária e suficiente para que $x^{S}$ e $x^{T}$ fossem adjacentes em $P_{P O C}(n)$, sendo $S$ e $T$ p.o.c. quaisquer de $D_{n}$.

Entretanto, encontramos exemplos como o que indicamos a seguir, onde [SAT] é conexo e $x^{S}$ não é adjacente a $x^{T}$ em $P_{P O C}(n)$. Este fato levou-nos à investigação do caso em que $S$ e $T$ estão relacionados pela relação de inclusão, já que neste caso $S \Delta T$ tem uma estrutura bastante simples, como pudemos verificar anteriormente.

Exemplo IV.8: Considere $T=<1,2, \ldots, n>$ uma ordem linear de $D_{n}$ e $\bar{T}=<n, n-$ $1, \ldots, 1>$ a ordem linear reversa à $T$, sendo $V_{n}=\{1,2, \ldots, n\}$. Note que $H=[T \Delta \bar{T}]$ é conexo. Seja $\alpha:=(i, i+1) \in T, \quad 1 \leq i<n$. Então,

$$
\begin{aligned}
& S=\langle 1,2, \ldots, i-1, i+1, i, i+2, \ldots, n\rangle \quad \mathrm{e} \\
& R=\langle n, n-1, \ldots, i+2, i, i+1, i-1, \ldots, 1\rangle
\end{aligned}
$$

são ordens lineares de $D_{n}$ e portanto p.o.c. de $D_{n}$. Considerando y o ponto médio do segmento que liga $x^{T}$ a $x^{\bar{T}}$ em $P_{P O C}(n)$, temos que:

$$
y=\frac{1}{2} x^{T}+\frac{1}{2} x^{\bar{T}}=\frac{1}{2} x^{S}+\frac{1}{2} x^{R} .
$$

Logo, $x^{T}$ nào é adjacente a $x^{\bar{T}}$ en Froc $(n)$.

Corolário IV.9: Seja $T$ uma p.o.c. de $D_{n}, n \geq 3$, tal que $T \neq A D_{n}$. Entâo $x^{T} \dot{e}$ adjacente: a $x^{A D_{n}}$ em $P_{P O C}(n)$.

Prova: Devido ao Teorema IV.7, é suficiente provarmos que $\left.H=\mid A D_{n} \backslash T\right]$ é conexo. Para isto, note que $V H=V D_{n}$ e considere $O L T=\left\langle T_{1}, \ldots, T_{k}\right\rangle, u \in V T_{i}$ e $v \in V T_{j}$, tais que $u \neq v$. Se $i<j(i>j)$ então $(v, u) \in A H((u, v) \in A H)$ e se $i=j$ existe em $H$ um caminho de u para $v$, passando por um vértice não pertencente a $V T_{i}$. 
Teorema IV.10: $\operatorname{DIAM}\left(P_{P O C}(n)\right)=2$, se $n \geq 3$.

Prova: Pelo Corolário IV.9, se $T$ é uma p.o.c. de $D_{n}$ distinta de $A D_{n}$, então $x^{T}$ é adjacente a $x^{A D_{n}}$ em $P_{P O C}(n)$. Logo,

$$
\operatorname{DIAM}\left(P_{P O C}(n)\right) \leq 2
$$

Para concluir o resultado, resta mostrar que existem p.o.c. S e $T$ de $D_{n}$, tais que $x^{\mathcal{S}}$ não é adjacente a $x^{T}$ em $P_{P O C}(n)$. Isto pode ser concluído do Exemplo IV.8.

Observação: Uma outra forma de provar que existem p.o.c. em $D_{n}, n \geq 3$, cujos vetores característicos não são adjacentes em $P_{P O C}(n)$, é usando o teorema devido a Young [1978] (veja também Hausmann [1980]) que caracteriza adjacência de dois vértices quaisquer no politopo da ordem linear, $P_{O L}(n)$. Com o objetivo de enunciar este resultado, faremos as seguintes convenções: se $x$ e $y$ são vetores característicos de ordens lineares de $D_{n}$, denotamos por $x \odot y$ (resp. $x \oplus y$ ) o vetor de zeros e uns obtido pelo cálculo da função lógica 'e' (resp. 'ou exclusivo') nos componentes de $x$ e $y$. Isto é,

$$
\begin{aligned}
& (x \odot y)_{i}=\left\{\begin{array}{ll}
1 & \text { se } x_{i}=y_{i}=1 \\
0 & \text { caso contrário }
\end{array}:\right. \\
& (x \oplus y)_{i}= \begin{cases}1 & \text { se } x_{i} \neq y_{i} \\
0 & \text { caso contrário }\end{cases}
\end{aligned}
$$

Teorema IV.11: Sejam $S$ e $T$ duas ordens lineares distintas de $D_{n}$. Construa o grafo $L(S, T)=(V L, A L)$ tal que:

$$
\begin{aligned}
& V L=\left\{\{u, v\}, u \in v \in V\left(D_{n}\right): z_{(u, v)}=1, \text { sendo } z=x^{S} \oplus x^{T}\right\} \epsilon \\
& A L=\left\{\{\{u, v\},\{v, w\}\}: z_{(u, w)}=1 \text { ou } z_{(w, u)}=1, \text { sendo } z=x^{S} \odot x^{T}\right\}
\end{aligned}
$$


Então $x^{S}$ e $x^{T}$ são adjacentes em $P_{O L}(n)$ se e somente se $L(S, T)$ for conexo.

Usando o teorema acima, podemos obter o resultado desejado. Para isto, considere uma ordem linear $T$ de $D_{n}$ e $\bar{T}$ seu reverso, $n \geq 3$. Então $x^{T} \oplus x^{\bar{T}}=1$ e $x^{T} \odot x^{\bar{T}}=0$. O grafo $L(T, \bar{T})$ consiste de $\left(\begin{array}{l}n \\ 2\end{array}\right)$ vértices e não possui arestas. Como $n \geq 3, L(T, \bar{T})$ é desconexo. Portanto, os vetores $x^{T}$ e $x^{\bar{T}}$ não são adjacentes em $P_{O L}(n)$. Como $P_{O L}(n)$ é uma face de $P_{P O C}(n)$, conforme vimos no capítulo III, segue que $x^{T}$ e $x^{\bar{T}}$ não são adjacentes em $P_{P O C}(n)$.

Finalizamos este capítulo apresentando um resultado que caracteriza adjacência de vértices em $P_{P O C}(n)$, se eles diferem em apenas dois componentes. Considere inicialmente a proposição:

Proposição IV.12: Sejam $S$ e $T$ p.o.c. de $D_{n}$ tais que $S \backslash T=\{a\}, T \backslash S=\{b\}$ e seja $H=[\{a, b\}]$. Então $H$ é desconexo se e somente se $S \cup T$ e $S \cap T$ são p.o.c. de $D_{n}$.

Prova: Suponha inicialmente que $S \cup T$ e $S \cap T$ são p.o.c. de $D_{n}$. A prova será por contradição. Para isto, considere $H$ conexo.

Se $b=\bar{a}$, entâo $(S \cap T) \cap\{a, \bar{a}\}=\emptyset$. Logo, $[S \cap T]$ nào é total e portanto $S \cap T$ nào é p.o.c. de $D_{n}$.

Suponha então que $b \neq \bar{a}$. Como $S$ e $T$ sào p.o.c., $\bar{a} \in T$ e $\bar{b} \in S$. De $b \neq \bar{a}$, segue que $\bar{a} \in S$ e $\bar{b} \in T$. Logo, $\{\bar{a}, \bar{b}\} \subseteq S \cap T$. Além disso, como $H$ é conexo, $[\{\bar{a}, \bar{b}\}]$ é conexo. Sejam $i$ e $j$ vértices de $V D_{n}$ que são extremos do caminho $\langle\bar{a}, \bar{b}\rangle$. Como $T$ é p.o.c., $\{\bar{a}, \bar{b}, b\} \subseteq T$ e $a \notin T$, segue que $(i, j) \in T$ se e somente se $(j, i) \notin T$. Seja $\alpha \in\{(i, j),(j, i)\}$ e suponha, s.p.g., que $\alpha \notin T$. Como $\alpha \neq a$, segue que $\alpha \notin S$ e portanto $\alpha \notin S \cup T$. De $\{a, \bar{a}, b, \bar{b}\} \subseteq S \cup T$ e $\alpha \notin S \cup T$, segue que $[S \cup T]$ nào é transitivo e portanto $S \cup T$ não é p.o.c. de $D_{n}$. 
Para provar a recíproca, suponha que $H$ é desconexo e vamos mostrar que $S \cup T$ e $S \cap T$ são p.o.c. de $D_{n}$. Como $S \subseteq S \cup T$ e $S$ é p.o.c., segue que [SUT] é total. Se $[S \cup T]$ não é transitivo, como $S$ e $T$ são p.o.c., existe um subgrafo $F$ de $[S \cup T]$ não transitivo tal que $|V F|=3$ e $\{a, b\} \subseteq A F$. Isto implica $H$ conexo, uma contradição. Logo, $S \cup T$ é uma p.o.c.

Claramente, $[S \cap T]$ é transitivo, pois $S$ e $T$ são p.o.c. Se $[S \cap T]$ não é total, como $S$ e $T$ são p.o.c., segue que $b=\bar{a}$. Neste caso, $H$ é conexo, uma contradição.

Portanto, $S \cap T$ e $S \cup T$ são p.o.c. de $D_{n}$ e a demonstração está completa.

Corolário IV.13: Sejam $S$ e T p.o.c. de $D_{n}$ e seja $H=[S \Delta T]$. Se $|A H|=2$, então $x^{S}$ e $x^{T}$ são adjacentes em $P_{P O C}(n)$ se e somente se $H$ é conexo.

Prova: Se $S \subseteq T$ ou $T \subseteq S$ o resultado é um corolário do Teorema IV.7. Suponha então que $S \backslash T=\{a\}$ e $T \backslash S=\{b\}$ e considere um ponto $y$ no interior do segmento que liga $x^{S}$ a $x^{T}$ em $P_{P O C}(n)$,

$$
y=\frac{1}{2} x^{S}+\frac{1}{2} x^{T}
$$

Suponha que $R_{1}, \ldots, R_{k}$ sejam p.o.c. de $D_{n}$, duas a duas distintas, tais que:

(a) $R_{i} \notin\{S, T\}, i=1, \ldots, k$.

(b) $y=\sum_{i=1}^{k} \lambda_{i} x^{R_{i}}, \quad \sum_{i=1}^{k} \lambda_{i}=1, \quad 0<\lambda_{i}<1$.

Neste caso, temos que se $\alpha \in S \cap T$, então

$$
1=\sum_{i=1}^{k} \lambda_{i} x_{\mathrm{cr}}^{R_{1}}
$$

e se $\alpha \in A D_{n} \backslash(S \cup T)$, então

$$
0=\sum_{i=1}^{k} \lambda_{i} x_{\alpha}^{R_{i}}
$$


Como $\lambda_{i}>0$ para todo $i=1, \ldots, k$, segue que

$$
x_{\alpha}^{R_{i}}=\left\{\begin{array}{ll}
1, & \text { se } \alpha \in S \cap T \\
0, & \text { se } \alpha \in A D_{n} \backslash(S \cup T)
\end{array} .\right.
$$

Portanto,

$$
S \cap T \subseteq R_{i} \subseteq S \cup T \text { para } i=1, \ldots, k
$$

donde podemos concluir que $k=2$ e podemos afirmar, s.p.g., que $R_{1}=S \cap T$ e $R_{2}=S \cup T$. Como $y=\frac{1}{2} x^{R_{1}}+\frac{1}{2} x^{R_{2}}$, concluímos que $x^{S}$ e $x^{T}$ não são adjacentes em $P_{P O C}(n)$ se e somente se $S \cup T$ e $S \cap T$ são p.o.c. de $D_{n}$. Pela Proposição IV.12, segue que $x^{S}$ e $x^{T}$ são adjacentes em $P_{P O C}(n)$ se e somente se $H$ é conexo. 


\section{ANEXO}

Teorema: A inequação-heptágono, apresentada na definiçäo III.19,

$$
4 x(C)-4 x(C O)+2 x(\bar{C})-2 x(\overline{C O})+x(D)-x(\bar{D}) \leq 9
$$

é válida para $P_{P O C}(7)$.

Prova: Denote a inequação-heptágono por $a^{T} x \leq 9$ e considere todas as operações aritméticas módulo 7. Como na definiçào III.19, suponha que $C:=\{(i, i+1): i=1, \ldots, 7\}$, $C O:=\{(i, i+2): i=1, \ldots, 7\}$ e $D:=\{(i, i+3): i=1, \ldots, 7\}$. Para facilitar o desenvolvimento posterior, escrevemos $a^{T} x \leq 9$ como $2 a_{1}^{T} x+a_{2}^{T} x \leq 9$, sendo:

$$
\begin{aligned}
& a_{1}^{T} x=2 x(C)-2 x(C O)+x(\bar{C})-x(\overline{C O}) \mathrm{e} \\
& a_{2}^{T} x=x(D)-x(\bar{D}) .
\end{aligned}
$$


Vamos provar o teorema, mostrando que se $H$ é um subdigrafo de $D_{7}$ tal que $x^{A H}$ é um vértice de $P_{P O C}(7)$, então $a^{T} x^{A H} \leq 9$. Para isto, será suficiente verificarmos que para um tal $H$, valem os seguintes lemas:

Lema 1: $a_{1}^{T} x \leq 6$ e $a_{2}^{T} x \leq 7$ são válidas para $P_{P O C}(7)$.

Lema 2: Se $a_{2}^{T} x^{A H} \leq-3$ ou $a_{2}^{T} x^{A H} \geq 6$, então $a^{T} x^{A H} \leq 9$.

Lema 3: Se $a_{1}^{T} x^{A H}=6$ então $a^{T} x^{A H} \leq 9$.

Lema 4: Se $4 \leq a_{2}^{T} x^{A H} \leq 5$ entâo $a_{1}^{T} x^{A H}<3$.

Lema 5: Se $2 \leq a_{2}^{T} x^{A H} \leq 3$ então $a_{1}^{T} x^{A H}<4$.

Lema 6: Se $0 \leq a_{2}^{T} x^{A H} \leq 1$ então $a_{1}^{T} x^{A H}<5$.

Note que, provados os lemas 1, 2 e 3, podemos restringir nossa atenção aos casos em que $3 \leq a_{1}^{T} x^{A H} \leq 5$ e $0 \leq a_{2}^{T} x^{A H} \leq 5$. Feito isto, a validade dos lemas 4,5 , e 6 garantirá a validade do teorema.

Nas demonstrações, devemos determinar as cardinalidades dos conjuntos $A H \cap Z$, para $Z \in \mathcal{C}$, sendo $\mathcal{C}=\{C, \bar{C}, C O, \overline{C O}, D, \bar{D}\}$. Para simplificar a notação, $S_{H}$ denota o conjunto $S \cap A H$, para $S \subseteq A\left(D_{7}\right)$. Todas as afirmaçòes a seguir são referentes a um digrafo $H \subseteq D_{7}$ tal que $x^{A H}$ é um vértice de $P_{P O C}(7)$.

Para auxiliar a prova desses lemas, vários resultados auxiliares serào provados ou enunciados.

Os cinco seguintes resultados serão utilizados na prova dos lemas. O primeiro resultado foi provado no capítulo III; os resultados $\mathrm{R} 2$ e $\mathrm{R} 3$ seguem da transitividade de $H$; o resultado R4 segue de $\mathrm{R} 1$; e o resultado $\mathrm{R} 5$ segue do fato de que $H$ é total. 
R1. As seguintes inequações são válidas para $P_{P O C}(7)$ :

$$
\begin{aligned}
& x(C)-x(C O)+x(\bar{C})-x(\overline{C O}) \leq 3, \\
& x(C)-x(C O) \leq 3 \quad \mathrm{e} \\
& x(\bar{C})-x(\overline{C O}) \leq 3 .
\end{aligned}
$$

R2. Se $Z \in \mathcal{C}$ e $\left|Z_{H}\right|=6$ então os valores da figura $A 1$ se verificam. A interpretação da primeira linha se refere ao caso $Z_{H}=C_{H}$ e é a seguinte: se $\left|C_{H}\right|=6$, então $\left|\bar{C}_{H}\right| \geq 1$, $\left|C O_{H}\right| \geq 5,\left|\overline{C O}_{H}\right| \geq 2,\left|D_{H}\right| \geq 4$ e $\left|\bar{D}_{H}\right| \geq 3$. Nos demais casos, a interpretação é análoga.

\begin{tabular}{c|ccccc}
$Z_{H}$ & $\geq 1$ & $\geq 5$ & $\geq 2$ & $\geq 4$ & $\geq 3$ \\
\hline$C_{H}$ & $\left|\bar{C}_{H}\right|$ & $\left|C O_{H}\right|$ & $\left|\overline{C O}_{H}\right|$ & $\left|D_{H}\right|$ & $\left|\bar{D}_{H}\right|$ \\
$\bar{C}_{H}$ & $\left|C_{H}\right|$ & $\left|\overline{C O}_{H}\right|$ & $\left|C O_{H}\right|$ & $\left|\bar{D}_{H}\right|$ & $\left|D_{H}\right|$ \\
$C O_{H}$ & $|\overline{C O}|$ & $\left|\bar{D}_{H}\right|$ & $\left|D_{H}\right|$ & $\left|\bar{C}_{H}\right|$ & $\left|C_{H}\right|$ \\
$\overline{C O}_{H}$ & $\left|C O_{H}\right|$ & $\left|D_{H}\right|$ & $\left|\bar{D}_{H}\right|$ & $\left|C_{H}\right|$ & $\left|\bar{C}_{H}\right|$ \\
$D_{H}$ & $\left|\bar{D}_{H}\right|$ & $\left|\bar{C}_{H}\right|$ & $\left|C_{H}\right|$ & $\left|C O_{H}\right|$ & $\left|C O_{H}\right|$ \\
$\bar{D}_{H}$ & $\left|D_{H}\right|$ & $\left|C_{H}\right|$ & $\left|\bar{C}_{H}\right|$ & $\left|C O_{H}\right|$ & $\left|C O_{H}\right|$ \\
\hline
\end{tabular}

Figura A1

R3. Se $Z \in \mathcal{C}$ e $\left|Z_{H}\right|=5$, então $\left|Z O_{H}\right| \geq 3$, sendo $Z O$ o conjunto das 2-cordas de $Z$. Para facilitar, listamos a seguir os pares $\left(Z_{H}, Z O_{H}\right)$ para $Z \in \mathcal{C}$ :

$$
\left(C_{H}, C O_{H}\right), \quad\left(\bar{C}_{H}, \overline{C O}_{H}\right), \quad\left(C O_{H}, \bar{D}_{H}\right), \quad\left(\overline{C O}_{H}, D_{H}\right), \quad\left(D_{H}, \bar{C}_{H}\right), \quad\left(\bar{D}_{H}, C_{H}\right)
$$

R4. Se $4 \leq a_{1}^{T} x^{A H} \leq 5$ então $\left|C_{H}\right|-\left|C O_{H}\right| \geq 1$. 
R5. As seguintes afirmações são verdadeiras:

i) se $\left|D_{H}\right| \leq 3$ então $a_{2}^{T} x^{A H} \leq-1$;

ii) se $\left|D_{H}\right|=4$ então $a_{2}^{T} x^{A H} \leq 1$;

iii) se $\left|D_{H}\right|=5$ então $a_{2}^{T} x^{A H} \leq 3$;

iv) se $\left|D_{H}\right|=6$ então $a_{2}^{T} x^{A H} \leq 5$.

Lema 1: $a_{1}^{T} x \leq 6$ e $a_{2}^{T} x \leq 7$ são válidas para $P_{P O C}(7)$

Prova do Lema 1: Obviamente $a_{2}^{T} x \leq 7$ é válida para $P_{P O C}(7)$. Uma vez que a inequação $a_{1}^{T} x \leq 6$ resulta da soma das inequações $x(C)-x(C O)+x(\bar{C})-x(\overline{C O}) \leq 3$ e $x(C)-x(C O) \leq 3$, sua validade segue de $\mathrm{R} 1$.

Lema 2: Se $a_{2}^{T} x^{A H} \leq-3$ ou $a_{2}^{T} x^{A H} \geq 6$, então $a^{T} x^{A H} \leq 9$.

Prova do Lema 2: Se $a_{2}^{T} x^{A H} \leq-3$, segue do Lema 1 que $a^{T} x^{A H} \leq 9$.

Observe que se $H$ possui um circuito $Z \in \mathcal{C}$, devido à transitividade de $H$, temos que $a^{T} x^{A H}=0$. Por outro lado, como $H$ é total, $a_{2}^{T} x^{A H} \geq 6$ implica $D \subseteq H$, e portanto $a^{T} x^{A H} \leq 9$, como queríamos demonstrar.

Como vimos na demonstração do lema acima, $\left|Z_{H}\right|=7$ para $Z \in \mathcal{C}$ implica $a^{T} x^{A H}=0$. Portanto, como $H$ é total, a partir daqui, vamos considerar $1 \leq\left|Z_{H}\right| \leq 6$ para $Z \in \mathcal{C}$.

Lema 3: Se $a_{1}^{T} x^{A H}=6$, entâo $a^{T} x^{A H} \leq 9$.

Prova do Lema 3: Como $a_{1}^{T} x^{A H}=2\left(\left|C_{H}\right|-\left|C O_{H}\right|\right)+\left|\bar{C}_{H}\right|-\left|\overline{C O}_{H}\right|=6$; e por R1 
temos que

$$
\left|C_{H}\right|-\left|C O_{H}\right|+\left|\bar{C}_{H}\right|-\left|\overline{C O}_{H}\right| \leq 3, \quad\left|C_{H}\right|-\left|C O_{H}\right| \leq 3 \quad \text { e } \quad\left|\bar{C}_{H}\right|-\left|\overline{C O}_{H}\right| \leq 3
$$

então

$$
\left|C_{H}\right|-\left|C O_{H}\right|=3 \text { e }\left|\bar{C}_{H}\right|-\left|\overline{C O}_{H}\right|=0
$$

- Se $\left|C O_{H}\right|=1$ então $\left|\overline{C O}_{H}\right| \geq 6$. Como estamos supondo $\left|\overline{C O}_{H}\right| \leq 6$, então $\left|\overline{C O}_{H}\right|=6$, e portanto, $\left|\bar{C}_{H}\right|=6$. Neste caso, de $\mathrm{R} 2$ segue que $\left|C O_{H}\right| \geq 2$, uma contradição.

- Se $\left|C O_{H}\right|=2$ então $\left|C_{H}\right|=5$, o que contraria R3.

- Se $\left|C O_{H}\right|=3$ então $\left|C_{H}\right|=6$. De R2 segue que $\left|C O_{H}\right| \geq 5$, uma contradição.

- Se $\left|C O_{H}\right| \geq 4$ então $\left|C_{H}\right| \geq 7$, contrariando a hipótese de que $\left|C_{H}\right| \leq 6$.

Portanto, $a_{1}^{T} x^{A H}=6$ não ocorre sob a hipótese de que para $Z \in \mathcal{C},\left|Z_{H}\right| \leq 6$. Como já vimos, para $Z \in \mathcal{C}$, se $\left|Z_{H}\right|=7$ então $a^{T} x^{A H} \leq 9$.

Veremos a seguir as demonstrações dos lemas 4, 5 e 6. Para isso, será usado seguinte roteiro:

Inicialmente, usando a hipótese, prova-se que $H$ possui um número grande de arestas em alguns dos seis circuitos $C, \bar{C}, C O, \overline{C O}, D$ ou $\bar{D}$. Constrói-se, a partir destas arestas o subdigrafo $\widehat{H}$, implicado pela transitividade. Em seguida, calcula-se $a_{1}^{T} x^{A \widehat{H}}$. A partir daí, mostra-se que:

"Para todo subdigrafo $H^{\prime}$ de $D_{7}$ tal que $\widehat{H} \subseteq H^{\prime}$ e $H^{\prime}$ satisfaz as hipóteses do lema, vale a tese."

Obviamente, $H=H^{\prime}$ para algum $H^{\prime}$ descrito acima. 
Para facilitar a notação, identificamos o digrafo $H$ com cada digrafo $H^{\prime}$, descrito acima, isto é, tentamos adicionar arestas ao grafo $\widehat{H}$, de forma a maximizar o valor de $a_{1}^{T} x$. Isto só será possivel se acrescentarmos arestas aos conjuntos $C_{\widehat{H}}$ e $\bar{C}_{\widehat{H}}$, uma vez que não podemos decrescer $\left|C O_{\widehat{H}}\right|$ ou $\left|\overline{C O}_{\widehat{H}}\right|$.

Lembramos aqui que, a partir deste ponto, estamos supondo $3 \leq a_{1}^{T} x^{A H} \leq 5$.

Lema 4: Se $4 \leq a_{2}^{T} x^{A H} \leq 5$ então $a_{1}^{T} x^{A H}<3$

\section{Prova do Lema 4:}

Pela hipótese, $4 \leq\left|D_{H}\right|-\left|\bar{D}_{H}\right| \leq 5$ e portanto $\left|D_{H}\right| \geq 4+\left|\bar{D}_{H}\right|$. Como $\left|\bar{D}_{H}\right| \geq 1$, temos que $\left|D_{H}\right| \geq 5$. Usando R5, concluímos que $\left|D_{H}\right| \neq 5$. Logo, $\left|D_{H}\right|=6$, donde,

$$
\left|\bar{D}_{H}\right| \leq 2
$$

Seja $\widehat{H}$ o subdigrafo de $D_{7}$, implicado por transitividade, a partir das arestas de $D_{H}$. Este digrafo pode ser visualizado na figura $\mathrm{A} 2$ abaixo. Em $\widehat{H}$, temos:

$$
\left|\bar{D}_{\hat{H}}\right|=1, \quad\left|\bar{C}_{\hat{H}}\right|=5, \quad\left|C_{\hat{H}}\right|=2, \quad\left|\overline{C O}_{\hat{H}}\right|=3 \quad \text { e } \quad\left|C O_{\hat{H}}\right|=4 .
$$

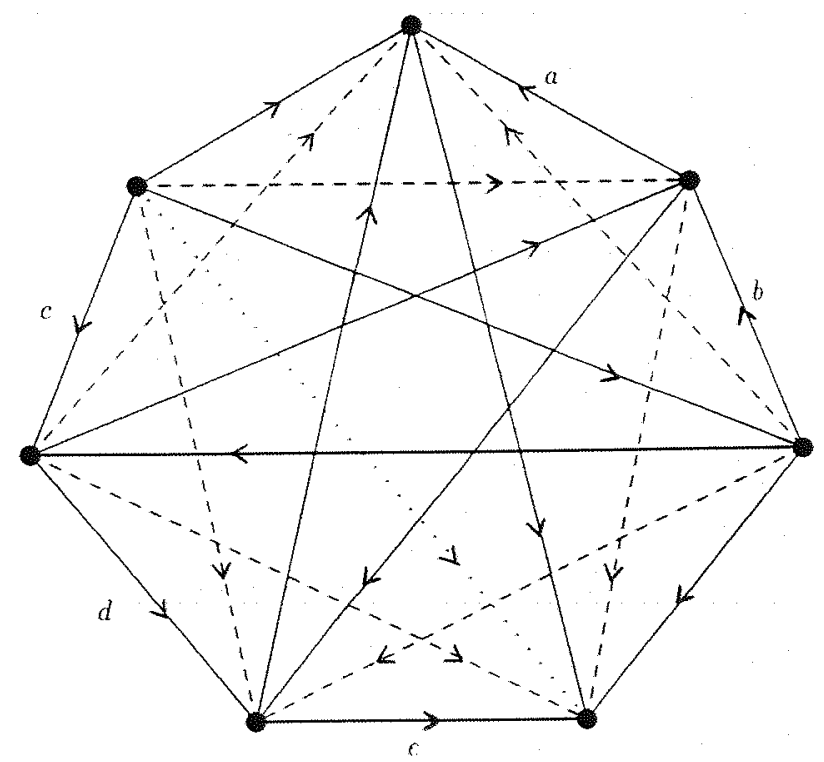

Figura A2 
Observe que se $\left|C_{H}\right|>2$, então $\left|\bar{D}_{H}\right|>2$, uma contradição à (III). Logo, não podemos aumentar $\left|C_{\widehat{H}}\right|$. Isto é suficiente para concluirmos a validade do lema, pois mesmo se $\left|\bar{C}_{H}\right|=6$, teríamos $a_{1}^{T} x^{A H} \leq-1$.

Lema 5: Se $2 \leq a_{2}^{T} x^{A H} \leq 3$ entâo $a_{1}^{T} x^{A H}<4$.

\section{Prova do Lema 5:}

Sendo $a_{2}^{T} x^{A H}>1$, segue de $R 5$ que $\left|D_{H}\right| \geq 5$. Temos dois casos a analisar:

Caso $1 .\left|D_{H}\right|=6$.

Neste caso, pela hipótese,

$$
\left|\bar{D}_{H}\right| \leq 4
$$

Conforme pode-se observar na figura $\mathrm{A} 2$, no subdigrafo $\widehat{H}$ de $D_{7}$ implicado pela transitividade a partir das arestas de $D_{H}$, temos:

$$
\left|\bar{D}_{\widehat{H}}\right|=1, \quad\left|\bar{C}_{\hat{H}}\right|=5, \quad\left|C_{\widehat{H}}\right|=2, \quad\left|\overline{C O}_{\hat{H}}\right|=3 \quad \text { e } \quad\left|C O_{\widehat{H}}\right|=4
$$

Suponha por absurdo que $a_{1}^{T} x^{A H} \geq 4$. Como $a_{1}^{T} x^{A H} \leq 5$, de R4, vem que $\left|C_{H}\right| \geq$ $\left|C O_{H}\right|+1$. Logo, $\left|C_{H}\right| \geq 5$. Mas, se $\left|C_{H}\right|>4$, então $C_{H}$ contém pelo menos duas arestas em um dos conjuntos $X=\{a, b\}$ ou $Y=\{c, d, \epsilon\}$, indicadas na figura A2. Isto implica $\left|\bar{D}_{H}\right| \geq 5$, uma contradiçào à inequação (IV).

Caso 2. $\left|D_{H}\right|=5$.

Neste caso,

$$
\left|\bar{D}_{H}\right| \leq 3
$$

Vamos analisar três subcasos: 
Caso 2.1. O comprimento máximo de um caminho em $D_{H}$ é 5 .

Caso 2.2. O comprimento máximo de um caminho em $D_{H}$ é 4 .

Caso 2.3. O comprimento máximo de um caminho em $D_{H}$ é 3 .

Caso 2.1. Considere o grafo $\widehat{H}$, representado na figura $\mathrm{A} 3$ abaixo, obtido a partir das arestas de $D_{H}$, acrescentando-se algumas arestas, devido à propriedade total.

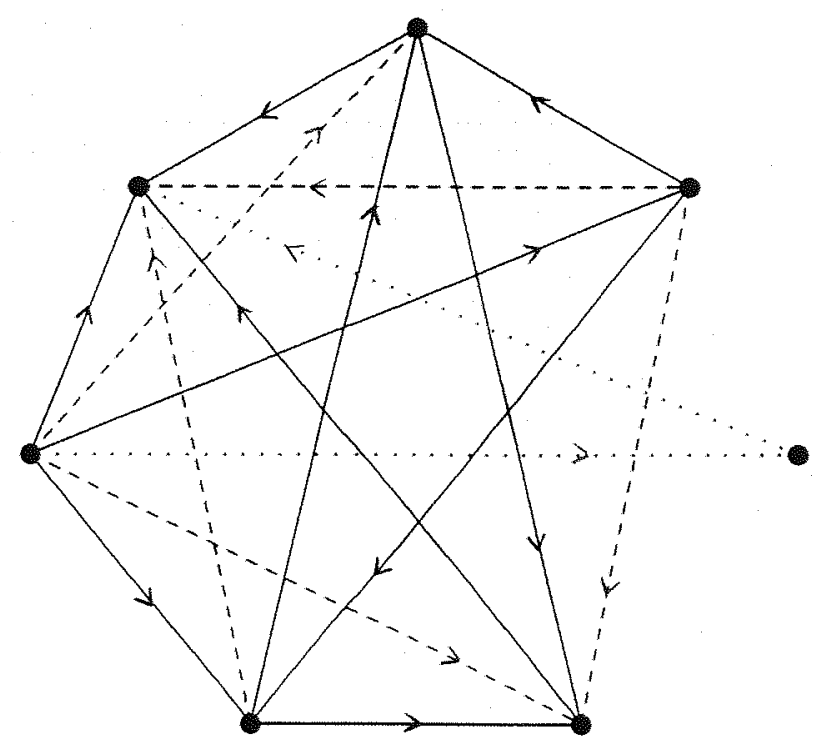

Figura A3

Observe que $\left|C_{H}\right|>3$ implica $\left|\bar{D}_{H}\right| \geq 4$, contradizendo (V). Logo, $\left|C_{H}\right| \leq 3$. Como $\left|C O_{H}\right| \geq 3$, segue que $\left|C_{H}\right|-\left|C O_{H}\right| \leq 0$. Usando R1, podemos concluir que $a_{1}^{T} x^{A H} \leq 3$.

Caso 2.2. Considere o grafo $\widehat{H}$, conforme descrição anterior, representado na figura $\mathrm{A} 4$ abaixo:

Note que $\left|C_{H}\right|>4$ implica $\left|\bar{D}_{H}\right|>3$, contrariando (V). Logo, $\left|C_{H}\right| \leq 4$.

Da observação da figura $\mathrm{A} 4$, concluímos que $\left|\bar{C}_{H}\right| \geq 3$ e $\left|C O_{H}\right| \geq 2$. 


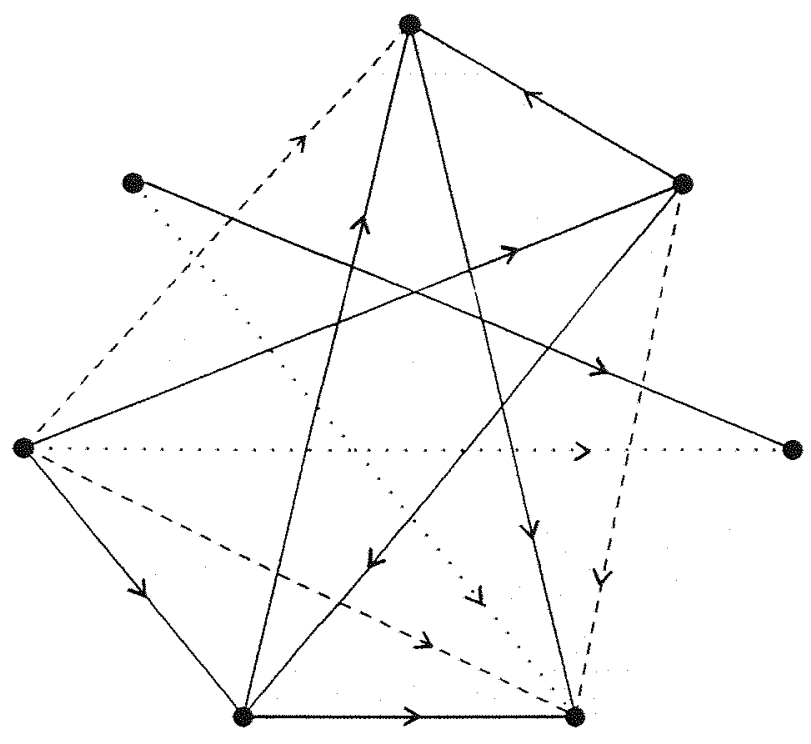

Figura A4

A inequação

$$
\left|\bar{C}_{H}\right|-\left|C O_{H}\right| \leq 1
$$

é verdadeira neste caso, isto é, a cada aresta distinta acrescentada ao conjunto $\bar{C}_{\hat{H}}$, devemos acrescentar pelo menos uma aresta distinta ao conjunto $C O_{\widehat{H}}$. A verificação deste fato fica a cargo do leitor.

Suponha por absurdo que $a_{1}^{T} x^{A H} \geq 4$. Como estamos nos restringindo a $a_{1}^{T} x^{A H} \leq 5$, devido a $\mathrm{R} 4$, basta considerarmos os casos em que $\left|C_{H}\right|-\left|C O_{H}\right| \geq 1$. De $\left|C_{H}\right| \leq 4 \mathrm{e}$ $\left|C O_{H}\right| \geq 2$, concluímos que devem ser analisados os seguintes casos:

Caso 2.2.1. $\left|C_{H}\right|=4$ e $\left|C O_{H}\right|=2$.

Neste caso, $\left|\bar{C}_{H}\right| \geq 3$. Das inequações (VI), $\left|\bar{C}_{H}\right| \geq 3$ e $\left|C O_{H}\right|=2$, segue que $\left|\bar{C}_{H}\right|=3$. Como $H$ é total, $\left|\overline{C O}_{H}\right| \geq 5$. Logo, $a_{1}^{T} x^{A H} \leq 2$.

Caso 2.2.2. $\left|C_{H}\right|=4$ e $\left|C O_{H}\right|=3$. 
Como $H$ é total, $\left|\bar{C}_{H}\right| \geq 3$ e $\left|\overline{C O}_{H}\right| \geq 4$.

Usando a inequação (VI), concluímos que $\left|\bar{C}_{H}\right| \leq 4$. Logo, $\left|\bar{C}_{H}\right|-\left|\overline{C O}_{H}\right| \leq 0$, donde $a_{1}^{T} x^{A H} \leq 2$.

Caso 2.2.3. $\left|C_{H}\right|=3$ e $\left|C O_{H}\right|=2$.

Como $H$ é total, $\left|\bar{C}_{H}\right| \geq 4$. Isto implica $\left|\bar{C}_{H}\right|-\left|C O_{H}\right| \geq 2$, uma contradição à inequação (VI).

Caso 2.3. Considere o grafo $\hat{H}$, conforme descriçâo anterior, representado na figura A5 abaixo.

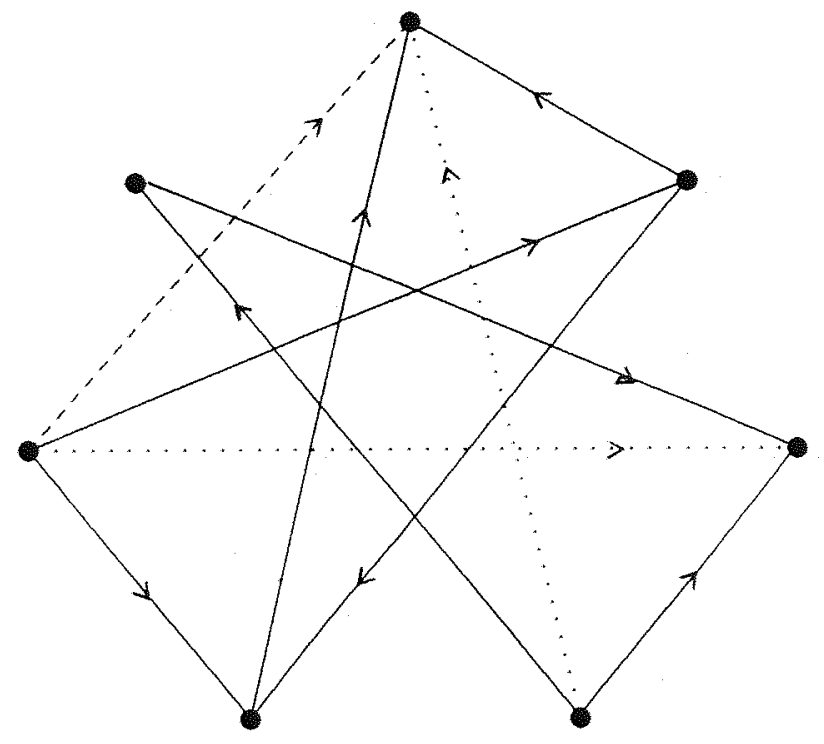

Figura A5

Observe que $\left|C_{H}\right|>4$ implica $\left|\bar{D}_{H}\right|>3$, uma contradição à inequação (V). Logo, $\left|C_{H}\right| \leq 4$. Neste caso, $\left|\bar{C}_{H}\right| \geq 3$ e $\left|C O_{H}\right| \geq 1$, conforme pode ser observado na figura. A5. A inequação

$$
\left|\bar{C}_{H}\right|-\left|C O_{H}\right| \leq 2
$$


é verdadeira, neste caso, e sua verificação fica a cargo do leitor.

Como no caso anterior, basta considerar os casos em que $\left|C_{H}\right|-\left|C O_{H}\right| \geq 1$. De $\left|C_{H}\right| \leq 4$ e $\left|C O_{H}\right| \geq 1$, concluímos que devem ser analisados os seguintes casos:

Caso 2.3.1. $\left|C_{H}\right|=4$ e $\left|C O_{H}\right|=1$.

Da propriedade total, segue que $\left|\bar{C}_{H}\right| \geq 3$ e $\left|\overline{C O}_{H}\right| \geq 6$. Como $\left|\overline{C O}_{H}\right| \leq 6$, concluímos que $\left|\overline{C O}_{H}\right|=6$. Usando (VII), concluímos que $\left|\bar{C}_{H}\right|=3$. Logo, $a_{1}^{T} x^{A H}=3$.

Caso 2.3.2. $\left|C_{H}\right|=4$ e $\left|C O_{H}\right|=2$.

Da propriedade total, segue que $\left|\bar{C}_{H}\right| \geq 3$ e $\left|\overline{C O}_{H}\right| \geq 5$.

Usando (VII), concluímos que $\left|\bar{C}_{H}\right| \leq 4$. Logo, $a_{1}^{T} x^{A H} \leq 3$.

Caso 2.3.3. $\left|C_{H}\right|=4$ e $\left|C O_{H}\right|=3$.

Da propriedade total, segue que $\left|\bar{C}_{H}\right| \geq 3$ e $\left|\overline{C O}_{H}\right| \geq 4$.

Usando (VII), concluímos que $\left|\bar{C}_{H}\right| \leq 5$. Logo, $a_{1} x^{A H} \leq 3$.

Caso 2.3.4. $\left|C_{H}\right|=3$ e $\left|C O_{H}\right|=1$.

Neste caso, $\left|\bar{C}_{H}\right| \geq 4$. Logo, $\left|\bar{C}_{H}\right|-\left|C O_{H}\right| \geq 3$, uma contradição à (VII).

Caso 2.3.5. $\left|C_{H}\right|=3$ e $\left|C O_{H}\right|=2$.

Então $\left|\bar{C}_{H}\right| \geq 4$ e $\left|\overline{C O}_{H}\right| \geq 5$. Usando (VII), segue que $\left|\bar{C}_{H}\right| \leq 4$. Logo, $a_{1}^{T} x^{A H} \leq 1$.

Caso 2.3.6. $\left|C_{H}\right|=2$ e $\left|C O_{H}\right|=1$.

Então $\left|\bar{C}_{H}\right| \geq 5$. Isto implica $\left|\bar{C}_{H}\right|-\left|C O_{H}\right| \geq 4$, uma contradiçào à (VII).

Com estes subcasos completamos a demonstraçào do Lema 5 . 
Lema 6: Se $0 \leq a_{2}^{T} x^{A H} \leq 1$ então $a_{1}^{T} x^{A H}<5$.

\section{Prova do Lema 6:}

Neste caso, basta provar que $a_{1}^{T} x^{A H} \neq 5$. Para isto, suponha, por absurdo, que $a_{1}^{T} x^{A H}=5$. Do resultado R4, segue que $\left|C_{H}\right|-\left|C O_{H}\right| \geq 1$. Este fato será usado freqüentemente nesta prova, sem uma referência explícita. A demonstração deste lema é exaustiva, pois analisamos 18 casos.

Caso 1. $2 \leq\left|C_{H}\right| \leq 3$.

Então, $1 \leq\left|C O_{H}\right| \leq 2$ e $0 \leq\left|C_{H}\right|-\left|C O_{H}\right| \leq 2$.

Da propriedade total, segue que $\left|\bar{C}_{H}\right| \geq 4$ e $\left|\overline{C O}_{H}\right| \geq 5$. Logo, $\left|\bar{C}_{H}\right|-\left|\overline{C O}_{H}\right| \leq 1$.

Como $a_{1}^{T} x^{A H}=5$, concluímos que $\left|C_{H}\right|-\left|C O_{H}\right|=2$ e $\left|\bar{C}_{H}\right|-\left|\overline{C O}_{H}\right|=1$. Portanto, $\left|C_{H}\right|=3$ e $\left|C O_{H}\right|=1$. Da propriedade total, concluímos que $\left|\overline{C O}_{H}\right| \geq 6$, donde $\left|\bar{C}_{H}\right|-\left|\overline{C O}_{H}\right| \leq 0$, uma contradição.

Caso 2. $\left|C_{H}\right|=4$.

Neste caso, devemos analisar os três subcasos:

Caso 2.1. $\left|C O_{H}\right|=3$.

Então, $\left|\overline{C O}_{H}\right| \geq 4$ e $\left|\bar{C}_{H}\right| \geq 3$. Logo, $a_{1}^{T} x^{A H} \leq 4$, um absurdo.

Caso 2.2. $\left|C O_{H}\right|=2$.

Neste caso, $6 \geq\left|\bar{C}_{H}\right| \geq 3$ e $6 \geq\left|\overline{C O}_{H}\right| \geq 5$.

Como $a_{1}^{T} x^{A H}=5,\left|C_{H}\right|=4$ e $\left|C O_{H}\right|=2$, segue que $\left|\bar{C}_{H}\right|=6$ e $\left|\overline{C O}_{H}\right|=5$.

A figura A6 representa o grafo $\widehat{H}$ implicado por transitividade, pelas arestas de $\bar{C}_{H}$. 


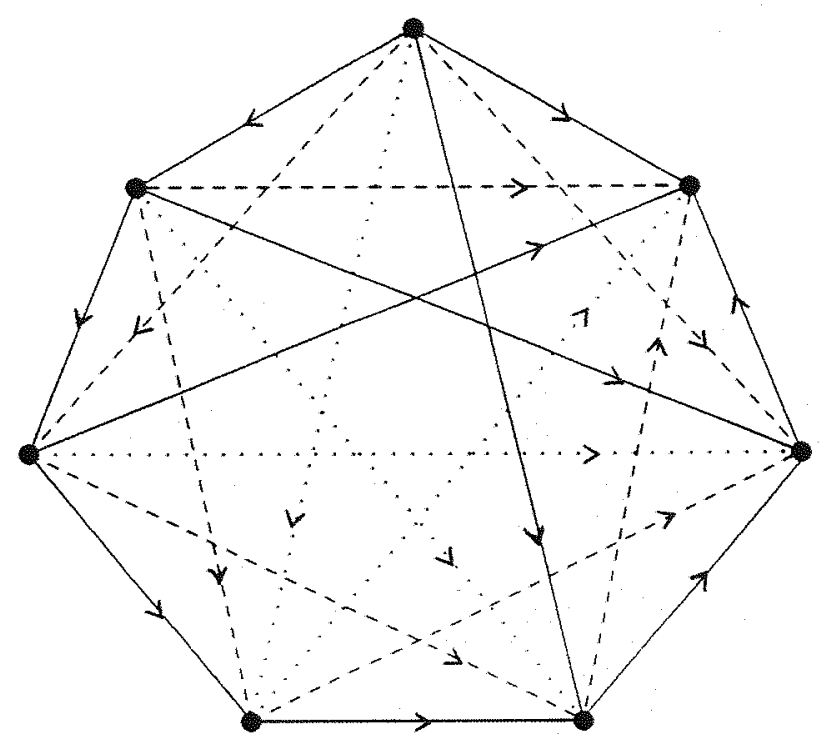

Figura $A 6$

Observe que $\left|D_{\widehat{H}}\right|=3,\left|\bar{D}_{\widehat{H}}\right|=4$ e $\left|C O_{\widehat{H}}\right|=2$. Como $0 \leq a_{2}^{T} x^{A H} \leq 1$, segue que $\left|D_{H}\right|-\left|\bar{D}_{H}\right| \geq 0$

Por outro lado, $\left|D_{\widehat{H}}\right|-\left|\bar{D}_{\hat{H}}\right|=-1$. A única forma de aumentar esta diferença é acrescentar arestas ao conjunto $D_{\hat{H}}$. Mas isto é impossivel, pois a cada nova aresta que acrescentamos a $D_{\hat{H}}$, devemos acrescentar uma nova aresta ao conjunto $C O_{\hat{H}}$.

Caso 2.3. $\left|C O_{H}\right|=1$.

Então $\left|\bar{C}_{H}\right| \geq 3$ e $\left|\overline{C O}_{H}\right| \geq 6$

Como $a_{1}^{T} x^{A H}=5$ e $\left|C_{H}\right|-\left|C_{H}\right|=3$, segue que $\left|\bar{C}_{H}\right|-\left|\overline{C O}_{H}\right|=-1$. De $\left|\overline{C O}_{H}\right|=6$ vem que $\left|\bar{C}_{H}\right|=5$

A figura A7 abaixo representa o grafo $\widehat{H}$ implicado por transitividade, pelas arestas de $\overline{C O}_{H}$. 


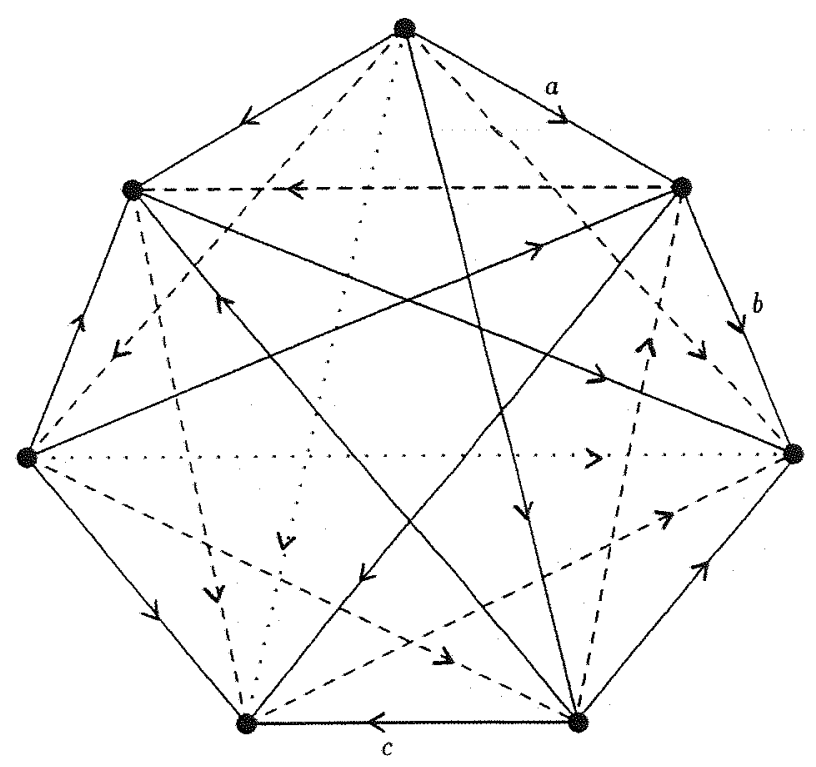

Figura A7

Observe que $\left|\bar{C}_{\widehat{H}}\right|=3$. Com o objetivo de obter o grafo $H$, devemos acrescentar duas arestas ao conjunto $\bar{C}_{\widehat{H}}$.

Por outro lado, nenhuma aresta do conjunto $\{\bar{a}, \bar{b}, \bar{c}\}$ pode ser acrescentada ao conjunto $\bar{C}_{\widehat{H}}$, pois o acréscimo de qualquer uma delas implica no acréscimo de uma nova aresta ao conjunto $C O_{\widehat{H}}$. Logo, este caso é impossível.

Caso 3. $\left|C_{H}\right|=5$.

Neste caso, segue que $\left|C O_{H}\right| \geq 3$, pelo resultado R3.

Como $\left|C_{H}\right|-\left|C O_{H}\right| \geq 1$, temos os seguintes subcasos:

Caso 3.1. $\left|C O_{H}\right|=4$.

Então $\left|\overline{C O}_{H}\right| \geq 3$ e $\left|\bar{C}_{H}\right| \geq 2$.

Se $\left|\bar{C}_{H}\right|=6$ então $\left|\overline{C O}_{H}\right| \geq 5$ e $a_{1}^{T} x^{A H} \leq 3$. 
Caso contrário, $a_{1}^{T} x^{A H} \leq 4$.

Em ambos os casos, $a_{1}^{T} x^{A H} \leq 4$, um absurdo.

Caso 3.2. $\left|C O_{H}\right|=3$.

Neste caso, $\left|\bar{C}_{H}\right| \geq 2$ e $\left|\overline{C O}_{H}\right| \geq 4$.

Como $a_{1}^{T} x^{A H}=5$ e $\left|C_{H}\right|-\left|C O_{H}\right|=2$, segue que $\left|\bar{C}_{H}\right|-\left|\overline{C O}_{H}\right|=1$. Temos aqui novamente dois subcasos:

Caso 3.2.1. $\left|C_{H}\right|=5,\left|C O_{H}\right|=3,\left|\bar{C}_{H}\right|=6$ e $\left|\overline{C O}_{H}\right|=5$.

Caso 3.2.2. $\left|C_{H}\right|=5,\left|C O_{H}\right|=3,\left|\bar{C}_{H}\right|=5$ e $\left|\overline{C O}_{H}\right|=4$.

\section{Caso 3.2.1}

Vamos analisar o grafo $\widehat{H}$ representado na figura A6, implicado por transitividade pelas arestas de $\bar{C}_{H}$.

Como $0 \leq a_{2} x^{A H} \leq 1$, segue que $\left|D_{H}\right|-\left|\bar{D}_{H}\right| \geq 0$.

$\operatorname{Em} \widehat{H},\left|D_{\widehat{H}}\right|=3, \quad\left|C O_{\widehat{H}}\right|=2, \quad\left|\bar{D}_{\widehat{H}}\right|=4 \quad$ e $\left|C_{\widehat{H}}\right|=1$.

Como $\left|D_{H}\right| \geq\left|\bar{D}_{H}\right|$ e $\left|\bar{D}_{H}\right| \geq\left|\bar{D}_{\hat{H}}\right|$, segue que

$$
\left|D_{H}\right| \geq 4 \text { e }\left|\bar{D}_{H}\right| \geq 4
$$

Conforme foi observado no caso 2.2 , a cada nova aresta que acrescentamos ao conjunto $D_{\widehat{H}}$, devemos acrescentar uma nova aresta ao conjunto $C O_{\hat{H}}$. Como $\left|C O_{H}\right|=3$ e $\left|C O_{\widehat{H}}\right|=2$, apenas uma aresta pode ser acrescentada ao conjunto $D_{\widehat{H}}$. Logo, $\left|D_{H}\right|=4$.

As inequações $\left|D_{H}\right|=4,\left|\bar{D}_{\hat{H}}\right|=4$ e $\left|D_{H}\right|-\left|\bar{D}_{H}\right| \geq 0$ implicam $\left|\bar{D}_{H}\right|=4$.

Devemos prosseguir, analisando o grafo representado pela figura A8, abaixo. É fácil ver que as arestas $a$ e $b$ devem ser acrescentadas ao conjunto $C_{\widehat{H}}$ pois se isto não ocorre, ao acrescentar quatro novas arestas ao conjunto $C_{\widehat{H}}$, teríamos necessariamente de acrescentar pelo menos duas novas arestas ao conjunto $C O_{\widehat{H}}$ e isto implicaria $\left|C O_{H}\right|>3$. 


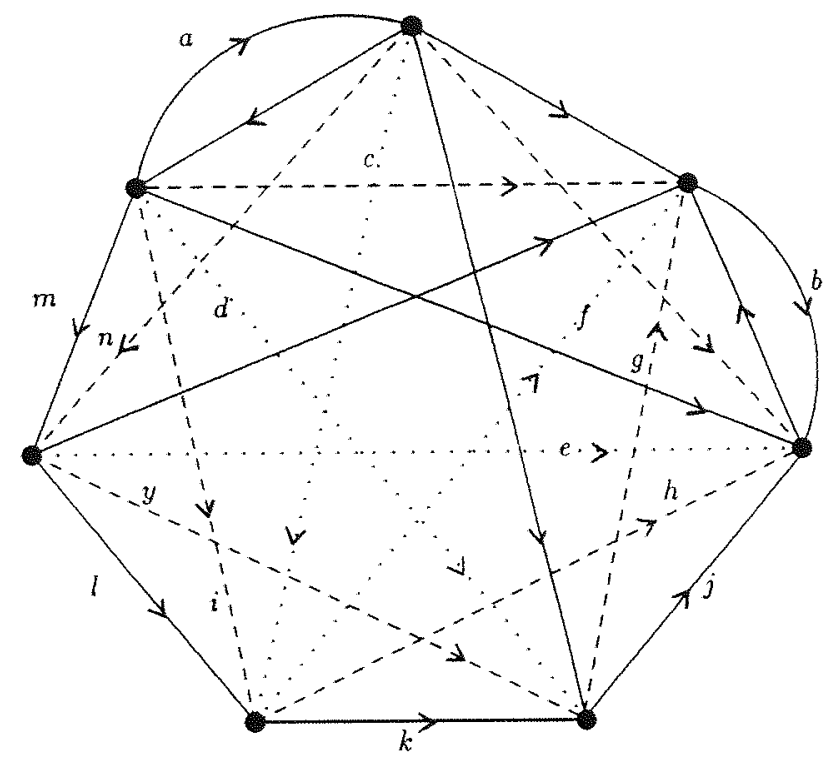

Figura A8

Como $\left|D_{H}\right|=4$, devemos acrescentar uma aresta ao conjunto $D_{\hat{H}}$. Observe que não podemos acrescentar as arestas $\bar{d}$ ou $\bar{\epsilon}$, pois isto implicaria no acréscimo de uma outra aresta ao conjunto $\bar{D}_{H}$.

Por outro lado, o acréscimo de $\bar{f}(\bar{c})$ ao conjunto $D_{\hat{H}}$ implica no acréscimo das arestas $\bar{g}$ e $\bar{h}(\bar{i}$ e $\bar{n})$ ao conjunto $C O_{\widehat{H}}$, o que é impossivel, pois $\left|C O_{H}\right|=3$.

\section{Caso 3.2.2}

As equações $\left|C_{H}\right|=5$ e $\left|C O_{H}\right|=3$ dào origem a dois grafos distintos, gerando dois subcasos.

Caso 3.2.2.1. O comprimento máximo de um caminho em $C_{H}$ é 3 .

Caso 3.2.2.2. O comprimento máximo de um caminho em $C_{H}$ é 4 .

Caso 3.2.2.1. A figura A9 representa o grafo $\widehat{H}$, implicado por transitividade pelas a- 
restas de $C_{H}$ e acrescido de algumas arestas implicadas pela propriedade total.

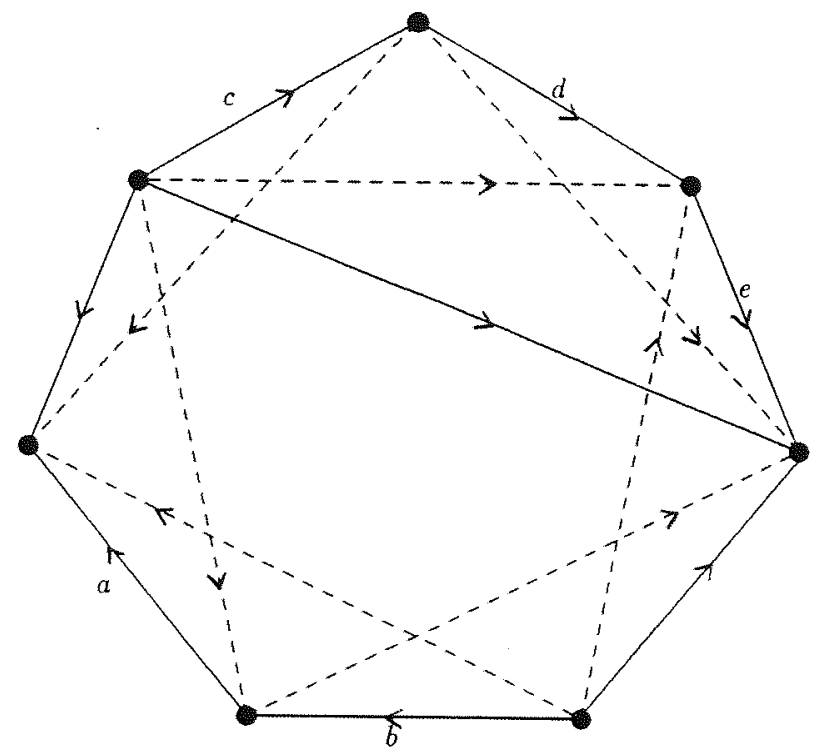

Figura A9

Como $\left|\bar{C}_{H}\right|=5$ e $\left|\bar{C}_{\hat{H}}\right|=2$, devemos acrescentar três arestas ao conjunto $\bar{C}_{\hat{H}}$. Se acrescentarmos as arestas $\bar{a}$ e $\bar{b}$ ou $\bar{c}$ e $\bar{d}$ ou $\bar{d}$ e $\bar{c}$, teremos que acrescentar uma aresta ao conjunto $\overline{C O}_{\hat{H}}$, o que é impossivel, pois $\overline{C O}_{H}=\overline{C O}_{\hat{H}}=4$.

Logo, as arestas $\bar{c}$ e $\bar{e}$ pertencem ao conjunto $\bar{C}_{H}$.

Novamente, devemos considerar dois subcasos:

Caso 3.2.2.1.1. A aresta $\bar{a}$ está em $\bar{C}_{H}$.

Caso 3.2.2.1.2. A aresta $\bar{b}$ está em $\bar{C}_{H}$.

Em ambos os casos, $a_{2}^{T} x^{A \widehat{H}}=-1$. Como $0 \leq a_{2}^{T} x^{A H} \leq 1$, devemos aumentar a diferença $\left|D_{\widehat{H}}\right|-\left|\bar{D}_{\widehat{H}}\right|$.

Considere inicialmente, o caso 3.2.2.1.1, representado na figura A10. Colocando as 


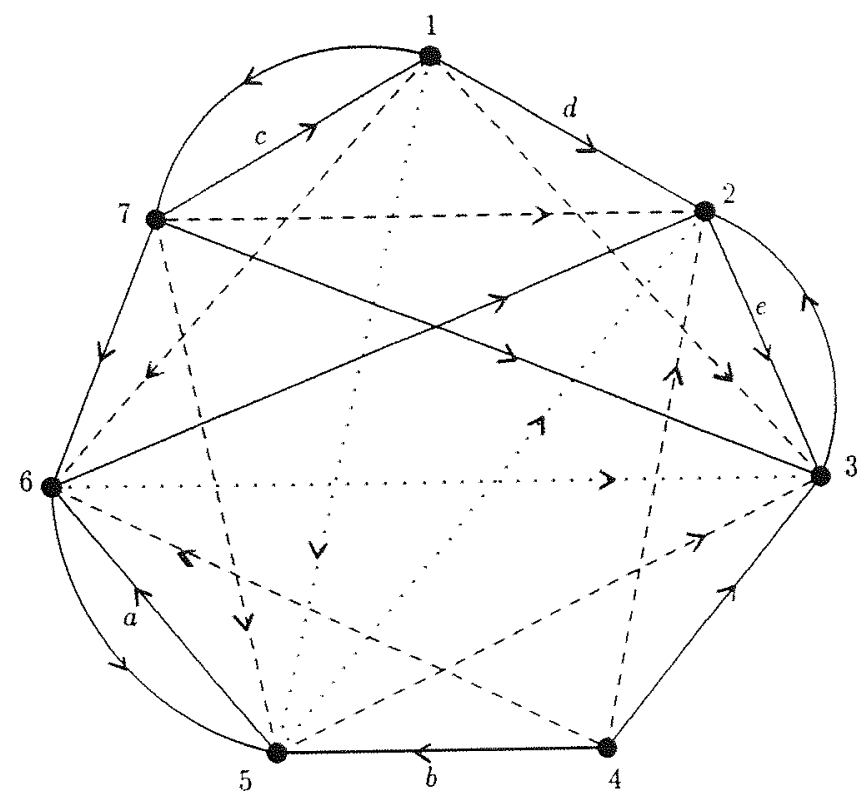

Figura A10

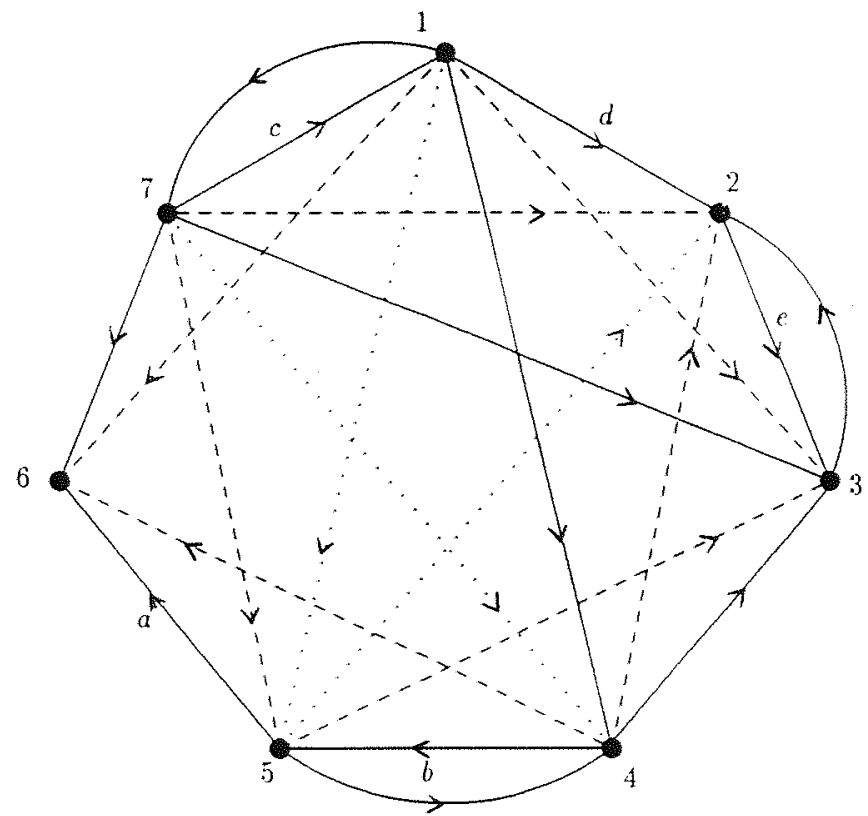

Figura A11 
arestas $(2,5),(3,6)$ ou $(5,1)$ em $D_{\widehat{H}}$, teremos $\left|C O_{H}\right|>3$, um absurdo. Resta então acrescentar as arestas $(4,7)$ ou $(1,4)$ a $D_{\widehat{H}}$. Mas o acréscimo de $(4,7)$ a $D_{\widehat{H}}$ implica no acréscimo de $(4,1)$ a $\bar{D}_{\widehat{H}}$ e o acréscimo de $(1,4)$ a $D_{\widehat{H}}$ implica no acréscimo de $(7,4)$ a $\bar{D}_{\hat{H}}$. Logo é impossivel aumentar $a_{2}^{T} x^{A \widehat{H}}$.

Para o caso representado pela figura A11, pode-se fazer uma verificação análoga a esta.

Caso 3.2.2.2. Considere, neste caso, a seguinte figura:

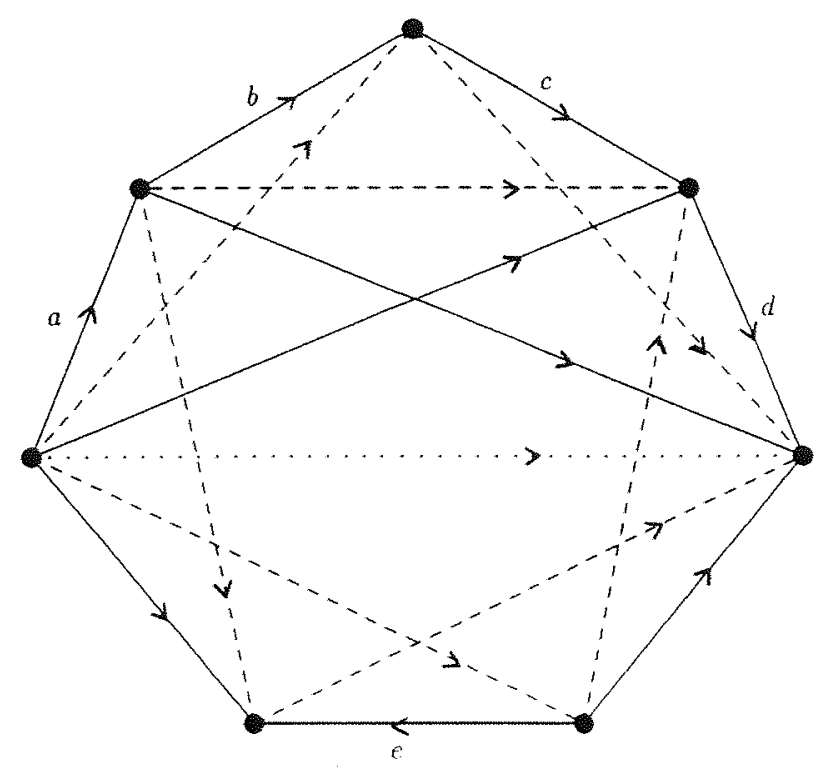

Figura A12

Como $\left|\bar{C}_{\hat{H}}\right|=2$ e $\left|\bar{C}_{H}\right|=5$, devemos acrescentar tres arestas ao conjunto $\bar{C}_{\hat{H}}$. Não é possível acrescentar duas arestas consecutivas pertencentes ao conjunto $\{\bar{a}, \bar{b}, \bar{c}, \bar{d}\}$, pois isto implicaria $\left|\overline{C O}_{H}\right|>4$.

Temos então três possibilidades:

Caso 3.2.2.2.1. As arestas $\bar{e}, \bar{b}$ e $\bar{d}$ estão em $\bar{C}_{H}$. 
Caso 3.2.2.2.2. As arestas $\bar{e}, \bar{a}$ e $\bar{c}$ estão em $\bar{C}_{H}$.

Caso 3.2.2.2.3. As arestas $\bar{e}, \bar{a}$ e $\bar{d}$ estão em $\bar{C}_{H}$.

Os três casos estão representadas nas figuras A13, A14 e A15 abaixo.

Nos três casos, $a_{1}^{T} x^{A \widehat{H}}=5$ e $a_{2}^{T} x^{A \widehat{H}}=-1$. A única forma de aumentar $a_{2}^{T} x^{A \widehat{H}}$ é acrescentar arestas ao conjunto $D_{\widehat{H}}$.

Nos dois primeiros casos, o acréscimo de qualquer aresta ao conjunto $D_{\widehat{H}}$ implica $\left|C O_{H}\right|>3$, uma contradição.

No caso 3.2.2.2.3, o acréscimo das arestas $(2,5)$ ou $(4,7)$ a $D_{\hat{H}}$ implica $\left|C O_{H}\right|>3$, uma contradição. Por outro lado, o acréscimo das arestas $(5,1),(3,6)$ ou $(1,4)$ a $D_{\widehat{H}}$ implica no acréscimo de $(4,1),(3,7)$ ou $(1,5)$ a $\bar{D}_{\widehat{H}}$, respectivamente. Logo, é impossivel aumentar $a_{2}^{T} x^{A \hat{H}}$.

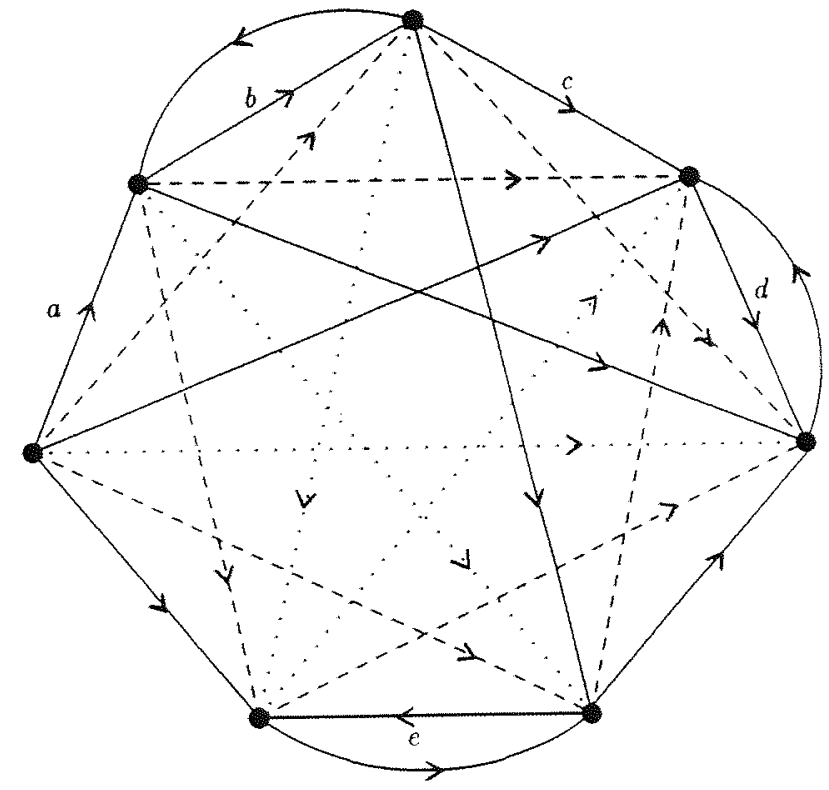

Figura A13 


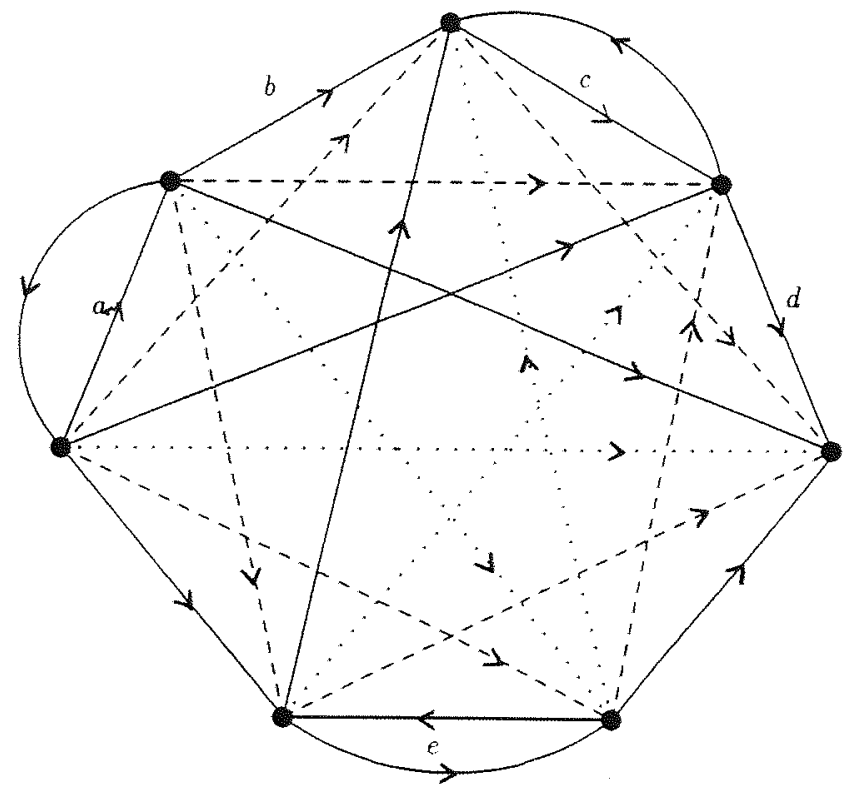

Figura A14

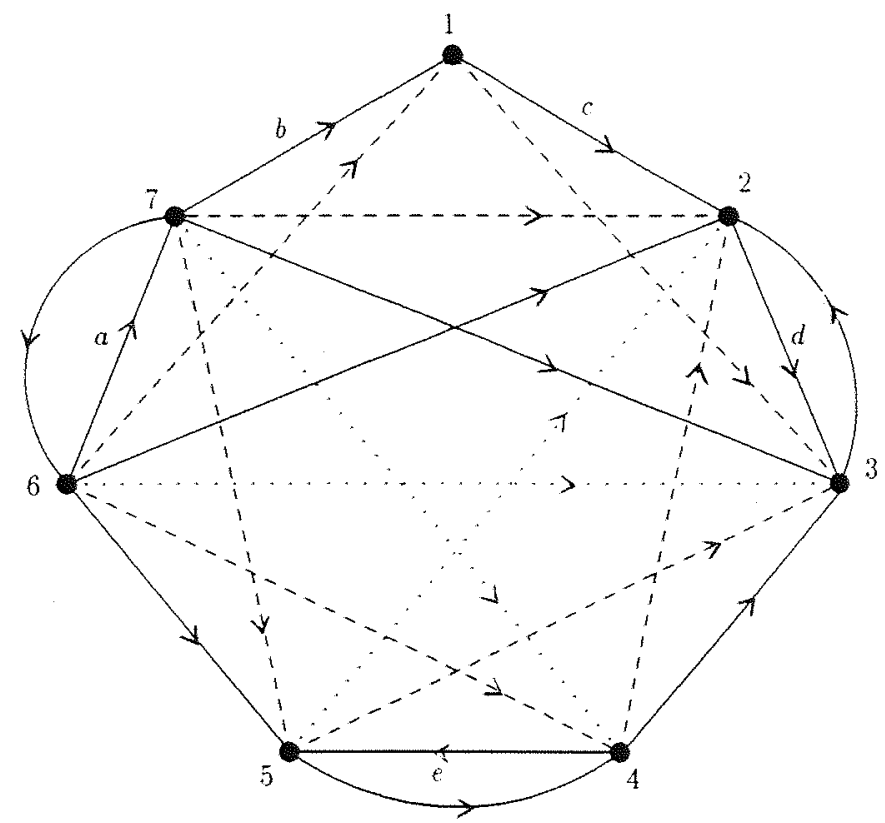

Figura A15 
Caso $4 .\left|C_{H}\right|=6$.

Da transitividade de $H$, segue que $\left|C O_{H}\right| \geq 5$. De $\left|C_{H}\right|-\left|C O_{H}\right| \geq 1,\left|C_{H}\right|=6$ e $\left|C O_{H}\right| \geq 5$, vem que $\left|C O_{H}\right|=5$. Como $H$ é total, concluímos que $\left|\overline{C O}_{H}\right| \geq 2$ e $\left|\bar{C}_{H}\right| \geq 1$. De $a_{1}^{T} x^{A H}=5$ e $\left|C_{H}\right|-\left|C O_{H}\right|=1$, segue que:

$$
\left|\bar{C}_{H}\right|-\left|\overline{C O}_{H}\right|=3
$$

Se $\left|\bar{C}_{H}\right| \leq 4$, então $\left|\bar{C}_{H}\right|-\left|\overline{C O}_{H}\right| \leq 2$, uma contradição à (VIII). Se $\left|\bar{C}_{H}\right|=5$, segue de R4 que $\left|\overline{C O}_{H}\right| \geq 3$, donde $\left|\bar{C}_{H}\right|-\left|\overline{C O}_{H}\right|<3$, uma contradição a (VIII).

Finalmente, se $\left|\bar{C}_{H}\right|=6$, segue da transitividade de $H$ que $\left|\overline{C O}_{H}\right| \geq 5$, donde $\left|\bar{C}_{H}\right|-$ $\left|\overline{C O}_{H}\right| \leq 1$, uma contradição à (VIII). Isto completa a prova do Lema 6 .

A prova do teorema segue dos lemas 1 a 7 , como já observamos anteriormente. 


\section{CONSIDERAÇÕES FINAIS}

Mencionamos aqui algumas questôes em aberto - de especial interesse para nós - que surgiram durante o desenvolvimento deste trabalho.

Como já observamos anteriormente, as classes de facetas apresentadas neste trabalho podem ser usadas no desenvolvimento de algoritmos que utilizam o método dos planosde-corte para resolver instâncias reais dos problemas aqui estudados. Para isso, é preciso resolver eficientemente o problema da separação para essas classes de facetas (cf. definimos na página 39).

É fácil verificar que para várias classes de facetas aqui apresentadas o problema da separaçâo pode ser resolvido em tempo polinomial. Não sabemos porém resolver este problema eficientemente para a classe das facetas dos circuitos ímpares com 2-cordas e para a classe das semi- $k$-cercas. É possivel que esses problemas sejam INI-completos e neste caso, seria interessante desenvolver heurísticas para resolvê-los. Neste contexto, um projeto que nos interessa sobremaneira é a implementação de um algoritmo do tipo branch and cut baseado nas classes de facetas aqui apresentadas.

Uma outra questão refere-se à figura apresentada na página 45. Mais precisamente, interessa-nos saber qual seria a resposta à seguinte questão: Existem facetas de $P_{P O}(n)$ que nâa são facetas de $P_{O P}(n)$ ou de $P_{P O C}(n)$ ?

Finalmente, um problema teórico interessante consiste em caracterizar adjacência de dois vértices quaisquer de cada um dos politopos $P_{P O}(n), P_{P O C}(n)$ e $P_{O P}(n)$. Conforme pode ser observado no capítulo IV, obtivemos apenas dois resultados caracterizando adjacência de vértices particulares de $P_{P O C}(n)$. 


\section{REFERENNCIAS}

Aho, A.V.\& Hopcroft, J.E. \& Ullman, J.D. [1974]: The Design and Analysis of Computer Algorithms, Addison-Wesley, Reading, MA, 1974.

Ascheuer, N.; Escudero, L.F., Grötschel, M. \& Stoer, M. [1990]: A cutting plane approach to the sequential ordering problem (with applications to job scheduling in manufacturing), Institut für Mathematik, Universität Augsburg, Report no 190 , 1990.

Barahona, F. \& Maccioni, E. [1982]: On the exact ground states of three-dimensional Ising spin glasses, Journal of Physics A: Math. Gen. 15 (1982), 1611-615.

Berge, C. [1973]: Graphs and Hypergraphs, North Holland, Amsterdam, 1973.

Bondy, J.A. \& Murty, U.S.R. [1976]: Graph Theory with Applications, Macmillan, London, 1976.

Brфndsted, A. [1983]: An Introduction to Convex Polytopes, Springer, New York, 1983.

Chvátal, V. [1983]: Linear Programming, Freeman, New York, 1983.

Cobham, A. [1965]: The intrinsic computational difficulty of functions, in: Y. Bar-Hillel (ed.), Proceedings of 1964 International Congress for Logic, Methodology and Phylosophy of Science, North-Holland, 1965, 24-30.

Crowder, H.P. \& Padberg, M.W. [1980]: Solving large scale travelling salesman problems to optimality, Management Science 26 (1980), 495-509.

Cook, S.A. [1971]: The complexity of theorem proving procedures, Proc. Third Annual ACM Symposium on Theory of Computing Association for Computing Machinery, New York, 1971, 151-158.

Dantzig, G.B. [1963]: Linear Programming and Extensions, Princeton University Press, Princeton, New Jersey, 1963.

Edmonds, J. [1965]: Paths, trees and flowers, Canad. J. Math. 17 (1965), 449-467. 
Garey, M.R. \& Johnson, D.S. [1979]: Computers and Intractability: A Guide to the Theory of NP-completeness, Freeman, San Francisco, 1979.

Grötschel, M. \& Lovász, L. \& Schrijver, A. [1981]: The ellipsoid method and its consequences in combinatorial optimization, Combinatorica 1 (1981), 169-197.

Grötschel, M. \& Jünger, M. \& Reinelt, G. [1984a]: A cutting plane algorithm for the linear ordering problem, Operations Research, vol. 32 (6), 1984, 1195-1220.

Grötschel, M. \& Jünger, M. \& Reinelt, G. [1984b]: Optimal triangulation of large realworld input-output matrices, Statistische Heft, 25 (1984) 261-295.

Grötschel, M. \& Padberg, M.W. [1985]: Polyhedral aspects of the travelling salesman problem I: theory, in: E.L. Lawler, J.K. Lenstra and A.H.G. Rinooy Kan (eds.), The Travelling Salesman Problem, Wiley, New York, 1985.

Grötschel, M. \& Wakabayashi, Y. [1989]: A Cutting Plane Algorithm for a Clustering Problem, Mathematical Programming 45 (1989),59-96.

Grünbaum, B. [1967]: Convex Polytopes, Wiley, New York, 1967.

Hausmann, D. [1980]: Adjacency on Polytopes in Combinatorial Optimization, Verlag, A. Hain, Meisenheim, 1980.

Jünger, M. [1985]: Polyhedral Combinatorics and the Acyclic Subdigraph Problem, (Research and Exposition in Mathematics 7) Heldermann Verlag, Berlin, 1985.

Karp, R.M. [1972]: Reducibility among combinatorial problems, in: R.E. Miller and J.W. Thatcher (eds.), Complexity of Computer Computations, pp. 85-103, Plenum Press, New York, 1972.

Lucchesi, C.L. [1979]: Introduçâa à Teoria dos Grafos, 120. Colóquio Brasileiro de Matemática, IMPA, 1979.

Minkowski, H. (1986): Geometrie der Zahlen (Erste Lieferung), Teubner, Leipzig, 1896 [reeditado: Chelsea, New York, 1953].

Motzkin, T.S. [1936]: Beiträge zur Theorie der linearen Ungleichungen (Inaugural Dissertation Basel), Azriel, Jerusalem, 1936 [Tradução Inglesa: Contributions to the Theory of Linear Inequalities, RAND Corporation Translation 22, Santa Monica, Cal., 1952 [reeditado em: Theodore S. Motzkin: Selected Papers (D. 
Cantor, B. Gordon and B. Rothschild, eds.), Birkhäuser, Boston, 1983), 1-80].

Padberg, M.W. \& Grötschel, M. [1985]: Polyhedral aspects of the travelling salesman problem II: computation, in: E.L. Lawler, J.K. Lenstra and A.H.G. Rinooy Kan (eds.), The Travelling Salesman Problem, Wiley, New York, 1985.

Padberg, M.W. \& Rao, M.R. [1974]: The travelling salesman problem and a class of polyhedra of diameter two, Mathematical Programming 7(1974), 32-45.

Papadimitriou, C.H. [1978]: The adjacency relation on the travelling salesman problem is NP-complete, Mathematical Programming 14 (1978), 312-324.

Papadimitriou, C.H. [1984]: Polytopes and Complexity, in: Progress in Combinatorial Optimization, Academic Press, 1984, 295-305.

Pulleyblank, W.R. [1983]: Polyhedral combinatorics, in: A. Bachem, M. Grötschel and B. Korte (eds.), Mathematical Programming, Bonn 1982 - The State of the Art, Springer Verlag, Berlin (1983), 312-345.

Reinelt, G. [1985]: The Linear Ordering Problem: Algorithms and Applications, (Research and Exposition in Mathematics 8) Heldermann Verlag, Berlin, 1985.

Schrijver, A. [1986]: Theory of Linear and Integer Programming, John Wiley \& Sons, New York, 1986.

Steinitz, E. [1916]: Bedingt konvergente Reihen und konvexe Systeme (Schluss), Journal für die reine und angewandte Mathematik 146 (1916), 1-52.

Szwarcfiter, J.L. [1984]: Grafos e Algoritmos Computacionais, Editora Campus, 1984.

Wakabayashi, Y. [1986]: Aggregation of Binary Relations: Algorithmic and Polyhedral Investigations, Tese, Universität Augsburg, 1986.

Weyl, H. [1985]: Elementare Theorie der Konvexen Polyeder, Commentarii Mathematici Helvetici 7, 1935, 290-306 [Tradução Inglesa: The elementary theory of convex polyhedra, in: Contributions to the Theory Games (H.W. Kuhn and A.W. Tucker, eds.), Princeton University Press, Princeton, N.J., 1950, 3-18].

Young, H.P. [1978]: On permutations and permutation polytopes, Mathematical Programming Study 8 (1978), 128-140. 


\section{Lista de símbolos e notação}

$\left(\begin{array}{l}n \\ k\end{array}\right) \quad$ número de combinaçôes de $n$ elementos tomados $k$ a $k$.

s.p.g. sem perda de generalidade .

$\lfloor x\rfloor$ o maior inteiro menor ou igual a $x$.

$|X| \quad$ cardinalidade de $X$.

$A \cap B \quad$ conjunto interseç̧ão de $A$ e $B$.

$A \cup B$ conjunto união de $A$ e $B$.

$A \backslash B \quad$ conjunto dos elementos que estão em $A$ e não estão em $B$.

$A \triangle B \quad(A \backslash B) \cup(B \backslash A)$.

$A \subseteq B \quad A$ é um subconjunto (subgrafo, subdigrafo) de $B$.

$A \subset B \quad A \subseteq B$ e $A$ é diferente de $B$.

$K_{n} \quad$ grafo completo com $n$ vértices.

$D_{n} \quad$ digrafo completo com $n$ vértices

$V_{n} \quad$ conjunto dos vértices de $D_{n}$.

$A_{n} \quad$ conjunto das arestas de $D_{n}$.

$V G$ conjunto dos vértices do grafo (digrafo) $G$.

$A G$ conjunto das arestas do grafo (digrafo) $G$.

$G[S],[S]$ subgrafo (subdigrafo) gerado pelo conjunto de vértices (ou arestas) $S$. 


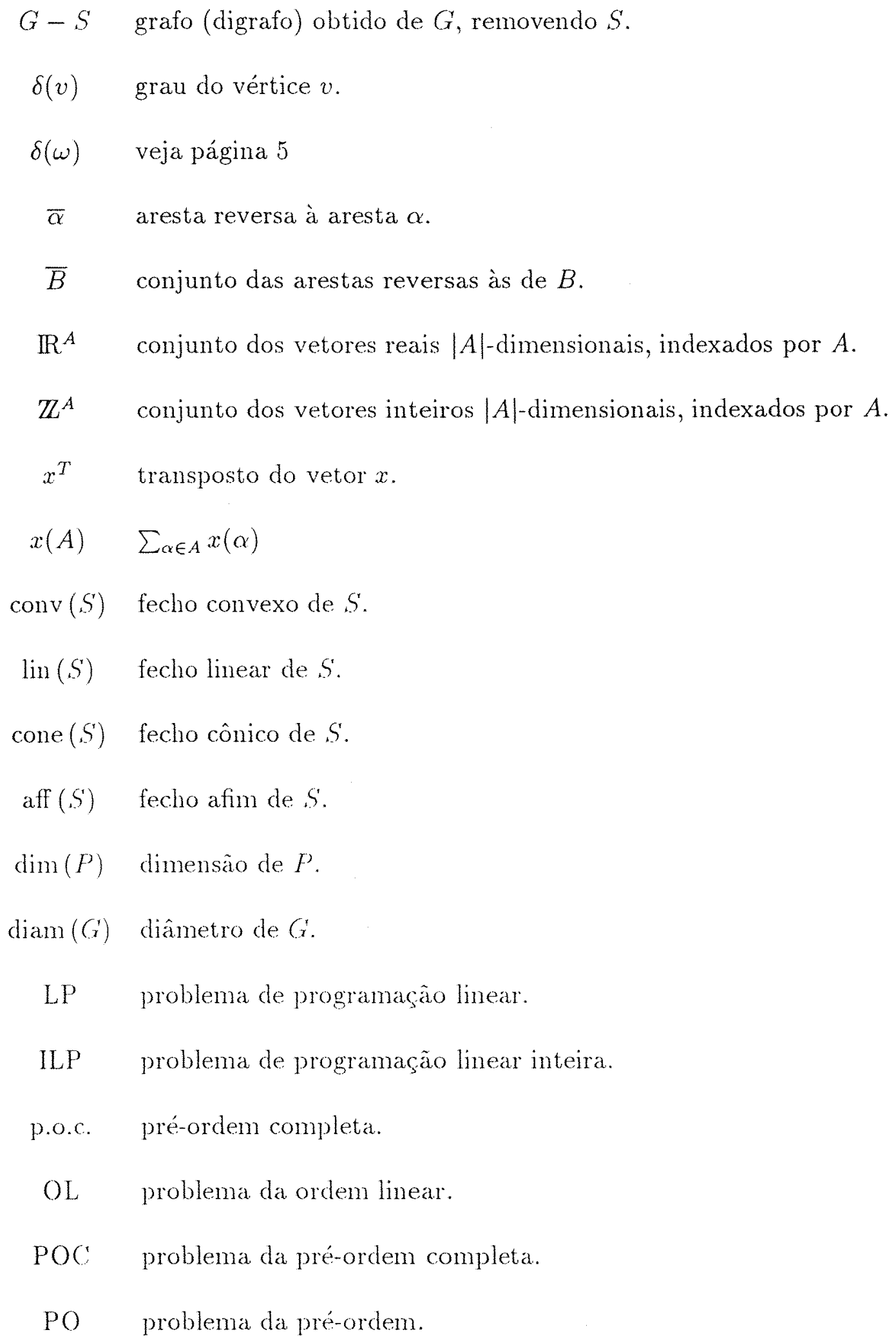


OP problema da ordem parcial.

$P_{O L}$ politopo da ordem linear.

$P_{P O C}$ politopo da pré-ordem completa.

$P_{P O}$ problema da pré-ordem.

$P_{O P}$ problema da ordem parcial.

FTR facetas dos triângulos.

FA facetas dos arcos paralelos.

FT facetas triviais.

FCC facetas dos circuitos ímpares com 2-cordas.

FP facetas dos pentágonos.

FH facetas dos heptágonos.

FSC facetas das semi-cercas. 


\section{Índice de Definições}

adjacência de vértices de um poliedro . . . . . . . . . . . . . 14

algoritmo . . . . . . . . . . . . . . . . . . . . . 17

- polinomial . . . . . . . . . . . . . . . . . . 18

aresta . . . . . . . . . . . . . . . . . 5

- de ligação . . . . . . . . . . . . . . . . . . . . . . 90

- local . . . . . . . . . . . . . . . . . . . . . . 90

- reversa . . . . . . . . . . . . . . . . . . . 6

caminho . . . . . . . . . . . . . . . . . . 6

- dirigido . . . . . . . . . . . . . . . . . . . 6

- hamiltoniano . . . . . . . . . . . . . . . . . . . 7

circuito . . . . . . . . . . . . . . . . 7

- dirigido . . . . . . . . . . . . . . . . . . . 7

- hamiltoniano . . . . . . . . . . . . . . . . 7

clique . . . . . . . . . . . . . . . . . 6

combinação afim . . . . . . . . . . . . . . . . . . . . 11

combinação convexa . . . . . . . . . . . . . . . . . . 11

combinaçào linear . . . . . . . . . . . . . . . . . . . . . 11

complexidade de tempo . . . . . . . . . . . . . . . . . . . 18

componentes . . . . . . . . . . . . . . . . . . . . . . . . 7

- fortemente conexos . . . . . . . . . . . . . 7

cone . . . . . . . . . . . . . . . . . . . 12

conjunto convexo . . . . . . . . . . . . . . . . . . . . . 12

conjunto das 2 -cordas . . . . . . . . . . . . . . . . . . . . . 7

conjunto das 3 -cordas . . . . . . . . . . . . . . . . . . . . . 7

conjunto reverso . . . . . . . . . . . . . . . . . . . . . . 6

corte . . . . . . . . . . . . . . . . 5

- orientado . . . . . . . . . . . . . . . . 5

descrição parcial de um politopo . . . . . . . . . . . . . . 39

diâmetro de um grafo . . . . . . . . . . . . . . . . . . . . . . 9

diâmetro de um politopo . . . . . . . . . . . . . . . . . 16

diferença simétrica . . . . . . . . . . . . . . . . . . 10

digrafo . . . . . . . . . . . . . . . . . . . 4

- acíclico . . . . . . . . . . . . . . . . . . 7

- anti-simétrico . . . . . . . . . . . . . . 8

- completo . . . . . . . . . . . . . . . . . . . . . 6

- condensado . . . . . . . . . . . . . . . . . . 7 
- conexo . . . . . . . . . . . . . . . . . . . . . . 7

- desconexo . . . . . . . . . . . . . . . . . . . . . . 7

- fortemente conexo . . . . . . . . . . . . . . . . . . 7

- gerado . . . . . . . . . . . . . . . . . . 7

- $k$-cerca . . . . . . . . . . . . . . . . . . 73

- semi-k-cerca . . . . . . . . . . . . . . . . . . . . . 75

- simétrico . . . . . . . . . . . . . . . . . . . 8

- simples . . . . . . . . . . . . . . . . . . . 5

- total . . . . . . . . . . . . . . . . . . . . 8

- transitivo . . . . . . . . . . . . . . . 8

digrafos isomorfos . . . . . . . . . . . . . . . . . . . 6

dimensão . . . . . . . . . . . . . . . . . . . . . . . 12

- completa . . . . . . . . . . . . . . . . . . . 13

distância . . . . . . . . . . . . . . . . . . . . . . . . . 9

eficiência de um algoritmo . . . . . . . . . . . . . . . . . . 17

emparelhamento . . . . . . . . . . . . . . . . . . . . . . 9

- perfeito . . . . . . . . . . . . . . . . . . . . . . . 9

esqueleto de um politopo . . . . . . . . . . . . . . . . . 16

esquema de codificação . . . . . . . . . . . . . . . . 18

extremos . . . . . . . . . . . . . . . . . . . . . . . . . 5

face . . . . . . . . . . . . . . . . . . . 14

- definida (induzida) por $a^{T} x \leq a_{0}$. . . . . . . . . . . . . 14

faces próprias . . . . . . . . . . . . . . . . . . . . . . 14

faceta. . . . . . . . . . . . . . . . . . 15

- das semi-k-cercas . . . . . . . . . . . . . . . . . . 47

- dos arcos paralelos . . . . . . . . . . . . . . . . . . 45

- dos circuitos impares com 2-cordas . . . . . . . . . . . 46

- dos heptágonos . . . . . . . . . . . . . . . . . . . 46

- dos pentágonos . . . . . . . . . . . . . . . . . . . . 46

- dos triângulos . . . . . . . . . . . . . . . . . . . 45

- triviais . . . . . . . . . . . . . . . . . . 46

fecho afim . . . . . . . . . . . . . . . . . . . . . 11

fecho connico . . . . . . . . . . . . . . . . . . . . . . . . 11

fecho convexo . . . . . . . . . . . . . . . . . . . . . . . 11

fecho linear . . . . . . . . . . . . . . . . . . . . . . . . 11

fecho transitivo . . . . . . . . . . . . . . . . . . . . . . . . . 9

fonte . . . . . . . . . . . . . . . . . . . . . . . 5

grafo . . . . . . . . . . . . . . . . . . . . . . . 9

- acíclico . . . . . . . . . . . . . . . . . . . . . . . 9

- dirigido . . . . . . . . . . . . . . . . . . . . . . 4

grau . . . . . . . . . . . . . . . . . . . . . . 5

hiperplano. . . . . . . . . . . . . . . . . . . 13 
inequação do $n$-circuito com 2 -cordas . . . . . . . . . . . . . 52

inequação do $n$-circuito duplo com 2 -cordas . . . . . . . . . . 60

inequação-faceta . . . . . . . . . . . . . . . . . . . . 15

inequação-heptágono . . . . . . . . . . . . . . . . 66

inequação induzida por um digrafo $G$. . . . . . . . . . . . . . . 40

inequação $k$-cerca . . . . . . . . . . . . . . . . . . . . . 74

inequação-pentágono . . . . . . . . . . . . . . . . . . . 59

inequação semi-k-cerca . . . . . . . . . . . . . . . . . . 76

inequação-triângulo . . . . . . . . . . . . . . . . . . . . . 47

inequação-válida . . . . . . . . . . . . . . . . . . 13

início . . . . . . . . . . . . . . . . . . . . . 5

método dos planos-de-corte . . . . . . . . . . . . 39

ordem linear . . . . . . . . . . . . . . . . . . . . . . . . . . . 8

ordem parcial . . . . . . . . . . . . . . . . . . . . . 8

passeio . . . . . . . . . . . . . . . . . . . . 6

- impar . . . . . . . . . . . . . . . . . . . . 7

$-\operatorname{par}$. . . . . . . . . . . . . . . . . 7

poliedro . . . . . . . . . . . . . . . . . . . . . 12

polinomialmente transformável . . . . . . . . . . . . . . . . 19

politopo . . . . . . . . . . . . . . . . . . . 13

- da ordem linear . . . . . . . . . . . . . . . . . . 37

- da ordem parcial . . . . . . . . . . . . . . . . . . . 37

- da pré-ordem . . . . . . . . . . . . . . . . . . . 37

- da pré-ordem completa . . . . . . . . . . . . . . . 37

posto . . . . . . . . . . . . . . . . 12

- afim . . . . . . . . . . . . . . . . . . 12

- coluna completo . . . . . . . . . . . . . . . . 12

- limha completo . . . . . . . . . . . . . . . . . . . . 12

pré-ordem . . . . . . . . . . . . . . . . . . . . 12

- completa . . . . . . . . . . . . . . . . 8

problema . . . . . . . . . . . . . . . . . . 17

- da ordem linear . . . . . . . . . . . . . . . . . 23

- da ordem parcial . . . . . . . . . . . . . . . . 23

- da pré-ordem . . . . . . . . . . . . . . . . . . . . . 23

- da pré-ordem completa . . . . . . . . . . . . . . . . 23

- da separação . . . . . . . . . . . . . . . . . . . . . 39

- de programação linear . . . . . . . . . . . . . . . . . . 16

- inteira . . . . . . . . . . . . . . . . . . 16

- relaxado . . . . . . . . . . . . . . 16

- do circuito hamiltoniano . . . . . . . . . . . . . . . . . . 19

- do subdigrafo acíclico . . . . . . . . . . . . . . . . . 26

- fácil . . . . . . . . . . . . . . . 18 
- $\mathbb{N} \mathbb{P}$-completo . . . . . . . . . . . . . . . . . . 20

- $\mathbb{N} \mathbb{P}$-difícil . . . . . . . . . . . . . . . . . 20

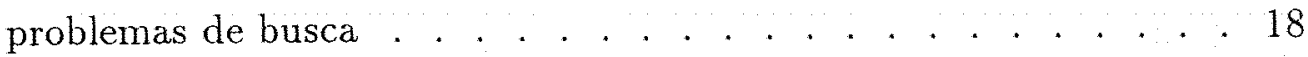

problemas de decisão

refinamento de uma pré-ordem completa

relação anti-simétrica

relação binária

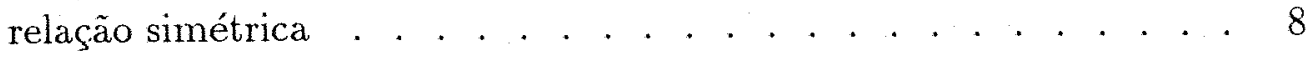

relação total . . . . . . . . . . . . . . . . . . . . . . . . . . . 8

relação transitiva . . . . . . . . . . . . . . . . . . . . . 8

semi-espaço . . . . . . . . . . . . . . . . . . . . . . . 13

solução ótima

solução viável . . . . . . . . . . . . . . . . . . . . . . . . 16

sorvedouro . . . . . . . . . . . . . . . . . . . . . . . 5

subconjunto dependente afim

subconjunto independente afim . . . . . . . . . . . . . . . . 12

subconjunto linearmente dependente . . . . . . . . . . . . . . 12

subconjunto linearmente independente . . . . . . . . . . . . 12

subdigrafo . . . . . . . . . . . . . . . . . . . . . . . . . . 5

- gerado . . . . . . . . . . . . . . . . 5

- gerador . . . . . . . . . . . . . . . . . 7

subespaço afim . . . . . . . . . . . . . . . . . . . . . . 12

subespaço linear . . . . . . . . . . . . . . . . . . . . . . 12

término . . . . . . . . . . . . . . . . . . . . . . . . 5

torneio . . . . . . . . . . . . . 7

triângulo . . . . . . . . . . . . . . . . . . . . . 7

turing-redutível . . . . . . . . . . . . . . . . . 20

vértice de um digrafo

vértice de um poliedro . . . . . . . . . . . . . . . . . . . . . . 14

vetor característico (de incidência) . . . . . . . . . . . . . 11

vetor unitário . . . . . . . . . . . . . . . . . . . . . . . 11 